University of Redlands

\title{
A Model to Identify Suitable Agricultural Sites after Climate Changes
}

A Major Individual Project submitted in partial satisfaction of the requirements for the degree of Master of Science in Geographic Information Systems

by

Joseph A. Koeller

Mark Kumler, Ph.D., Committee Chair

Mark Stewart, M.S.

August 2011 
A Model to Identify Suitable Agricultural Sites after Climate Changes

Copyright (C) 2011

by

Joseph A. Koeller 
The report of Joseph A. Koeller is approved.

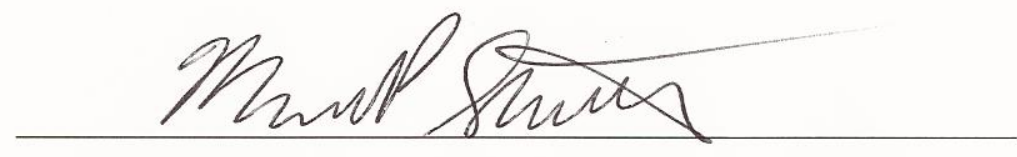

Mark Stewart, M.S.

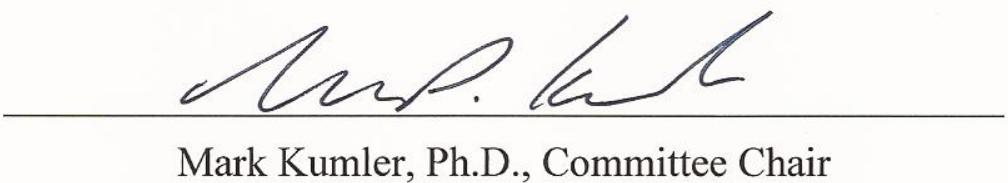

August 2011 



\section{Acknowledgements}

I acknowledge the Marvin R. Morrison Family Trust Fellowship program for providing substantial funding to attend the MS-GIS Program at the University of Redlands. This generous contribution allowed me to attend the program, which would have otherwise been in doubt. As a result of attending the MS-GIS program, I was able to apply my research interests in climate change to the Morrison interest in agriculture. For that I credit the Marvin R. Morrison Family Trust Fellowship for those research accomplishments as well as the personal benefits I receive as a result of obtaining a Master of Science degree.

I also acknowledge my father David J. Koeller, whose personal love of geography and travel sparked my interest for the subject matter at a young age. My father would routinely take my family on road-trip vacations almost every summer, further back in the past before I can even remember. In the car I liked to gaze out of the window and observe the landscape as well as meticulously follow along on road maps he would pick up for me at highway rest areas in each new state we entered along the way. To this day, I love the subject of geography and have a personal hobby of road tripping across the United States.

Additionally I acknowledge my mother Linda A. Koeller, who from the very start of my educational background in Sacred Heart Elementary worked with me almost on a daily basis through the struggles I had with class material. She was the only person who would continuously assure me that I could do better than what my grades reflected, when in elementary school. I have spent countless hours and nights working on coursework and studying for exams since to prove to her and myself that she was right all along. I would be awarded an honor or degree in my collegiate years and think of her, remembering that she inspired a struggling $\mathrm{C}$ student to earn a Master of Science degree before the age of 25 . 



\begin{abstract}
A Model to Identify Suitable Agricultural Sites after Climate Changes

by

Joseph A. Koeller

A model was developed for county planners to use to identify potential locations for agricultural and environmental vegetation planning and development. The production of the Hawaiian Vegetation Model involve simulation changes in precipitation and temperature on the island to determine what effect these variables have on the Hawaiian vegetation. The variables of temperature and precipitation were chosen as inputs to the vegetation model because they are used in numerous climate change models. The resulting agricultural and environmental vegetation scenarios from the precipitation and temperature inputs identify the best potential locations for agricultural and environmental vegetation planning and development.
\end{abstract}





\section{Table of Contents}

Chapter 1 - Introduction ................................................................................................ 1

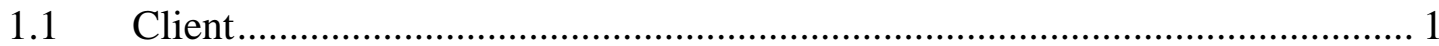

$1.2 \quad$ Problem Statement ................................................................................ 1

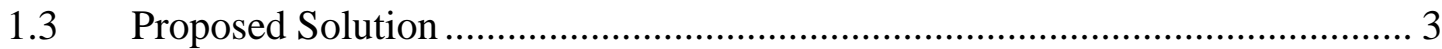

1.3.1 Goals and Objectives …………………………..................................... 3

1.3.2 Scope

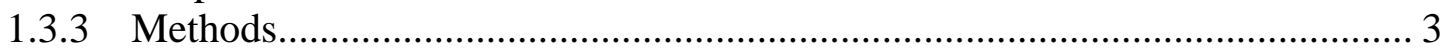

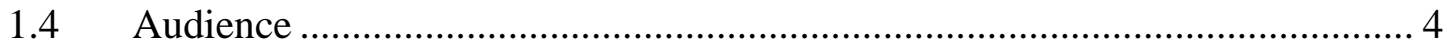

1.5 Overview of the Rest of this Report ........................................................... 4

Chapter 2 - Background and Literature Review ............................................................... 7

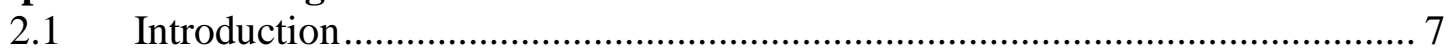

2.2 The GIS Data Model............................................................................. 7

2.3 Relationship Between Vegetation and Climate …………………………...... 7

2.4 The Climate Change Model .................................................................. 12

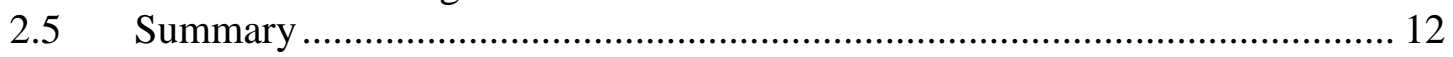

Chapter 3 - Systems Analysis and Design...................................................................... 15

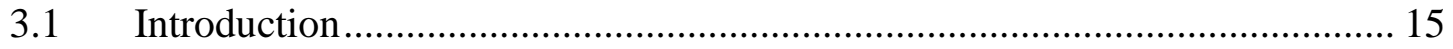

3.2 Problem Statement ........................................................................... 15

3.3 Requirements Analysis ……………………….................................... 16

3.3.1 Functional Requirements ........................................................................ 16

3.3.2 Non-Functional Requirements ................................................................. 16

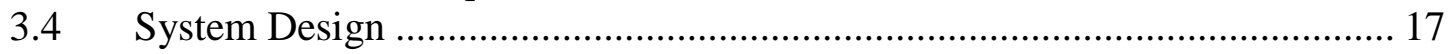

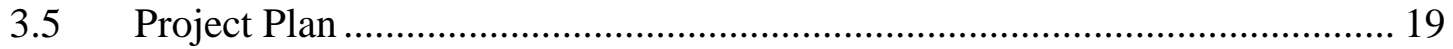

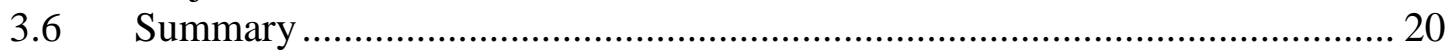

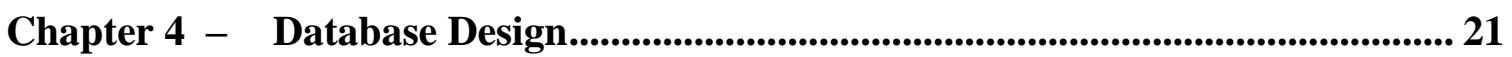

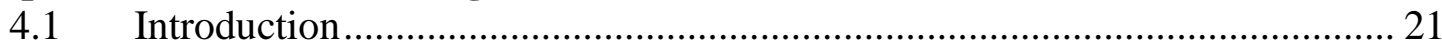

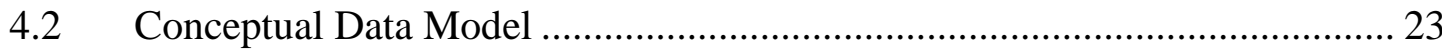

$4.3 \quad$ Logical Data Model ……………………………............................. 24

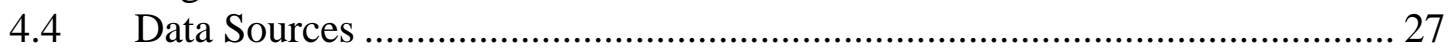

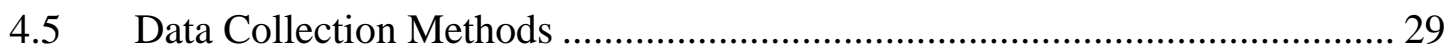

4.6 Data Scrubbing and Loading ……………………................................... 30

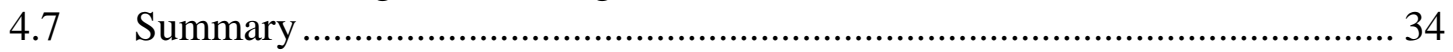

Chapter 5 - Implementation............................................................................................. 37

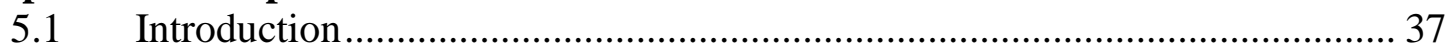

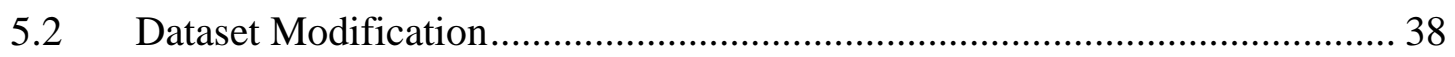

5.2.1 Climate Dataset Modification ................................................................... 38

5.2.2 Elevation Dataset Modification ................................................................... 41

5.2.3 Dataset Modification Summary …………………................................... 45

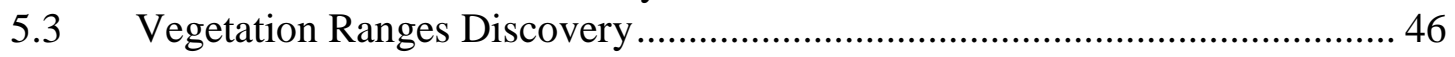

5.3.1 Vegetation Dataset Comparison .................................................................... 46

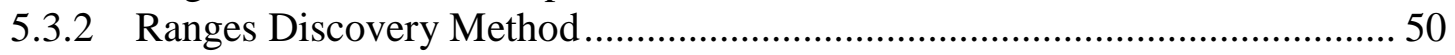

5.3.3 Ranges Discovery Summary …………………………………………..... 50 
$5.4 \quad$ Data Model Preparatory Work ................................................................. 51

5.4.1 Vegetation Model Preparatory Work ............................................................. 51

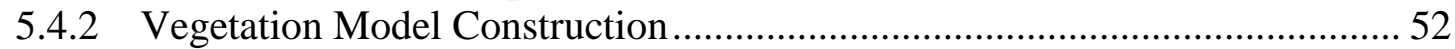

5.4.3 Vegetation Model Script and Dialog ........................................................ 54

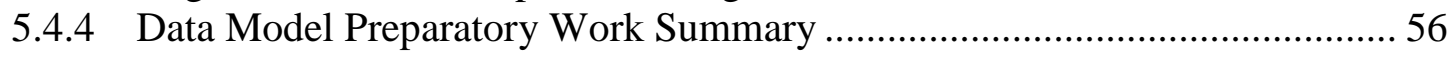

5.5 Comparison of Interpolation Algorithms .............................................. 57

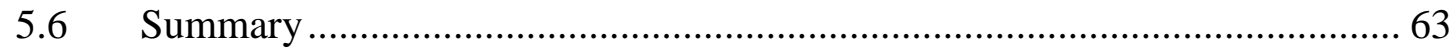

Chapter 6 - Results and Analysis............................................................................ 65

6.1 What Are the Users Allowed to do with these Models? .................................65 65

6.2 What Happens When the User Runs the Models? ....................................... 66

6.3 What Process Went Right? What Process Went Wrong? .............................. 78

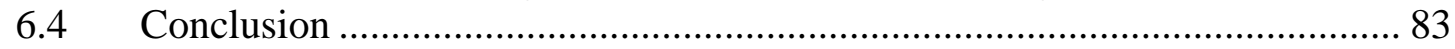

Chapter 7 - Conclusions and Future Work ............................................................... 85

7.1 Introduction and Project Goals Review …............................................... 85

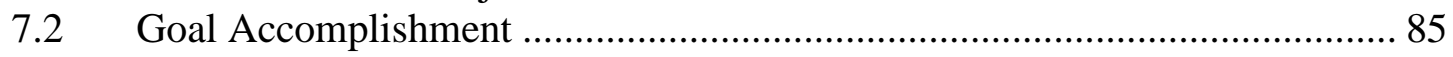

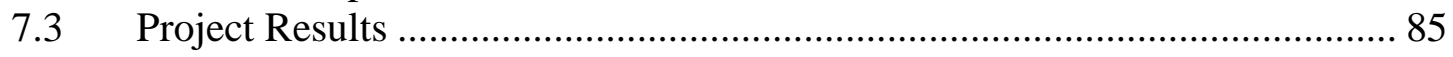

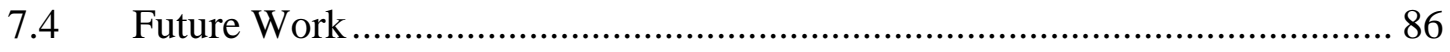

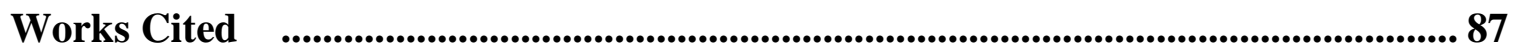

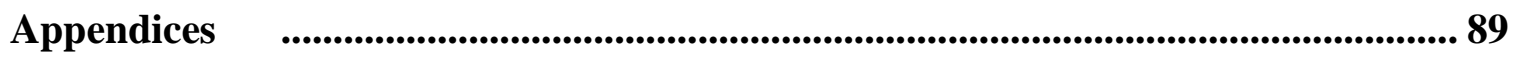




\section{Table of Figures}

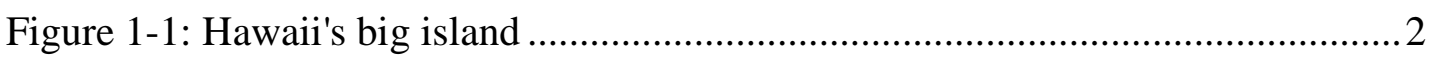

Figure 1-2: Vegetation model instruction ............................................................. 4

Figure 2-1: World climates. Source: www.blueplanetbiomes.org.............................. 8

Figure 2-2: Hawaii's global position in terms of latitude and longitude.......................9

Figure 2-3: Hawaii's Annual Precipitation Averages .................................................. 10

Figure 2-4: Hawaiian Minimum (left) and Maximum (right) Temperature ................11

Figure 2-5: Hawaiian Elevation .......................................................................... 11

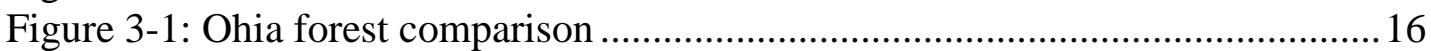

Figure 3-2: Vegetation map and technology mockup ............................................... 18

Figure 4-1: Hawaii's northeast coast. Source: Hawaii Statewide GIS Program .........24

Figure 4-2: Attribute Specification of Vegetation Type ............................................26

Figure 4-3: Vegetation Land Use Dataset.............................................................29

Figure 4-4: NRCS_CropForestRangelands attribute table .........................................31

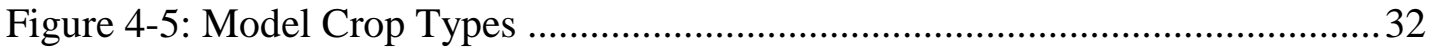

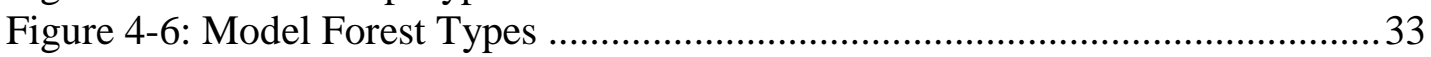

Figure 5-1: Vector formats of minimum (top) and maximum (bottom) temperature 40

Figure 5-2: Vector format of precipitation.............................................................4

Figure 5-3: Metadata of the 100-foot contours elevation dataset ................................43

Figure 5-4: Vector format of the resampled 1000-meter DEM ……............................44

Figure 5-5: Comparison between the resampled datasets............................................4 44

Figure 5-6: Attributes of the aggregated climate and elevation dataset.......................45

Figure 5-7: Select by attributes and select by location tool working in conjunction. 47

Figure 5-8: The SPSS output of the percentile points percent values of coffee .........48

Table 5-1: Summary statistics for each dataset and each vegetation type ...................48

Figure 5-9: Attribute table of the aggregated dataset..................................................51

Figure 5-10: Possible polygons (left) and selected polygons (right) …………...........53

Figure 5-11: Vegetation script dialog (left) and vegetation model expression (top

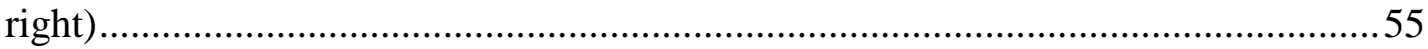

Figure 5-12: Selecting a vegetation type (top) and a habitat type (bottom) ...............56

Figure 5-13: Comparison of IDW and Kriging Surfaces ..........................................58

Figure 5-14: Minimum Temperature Interpolation Inputs..........................................59

Figure 5-15: Maximum Temperature Interpolation Inputs ...........................................60

Figure 5-16: Precipitation Interpolation Inputs........................................................61

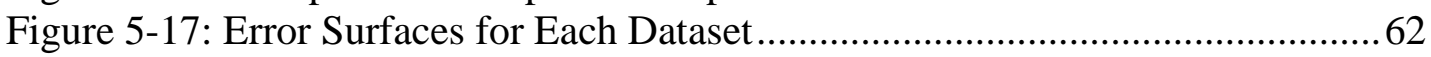

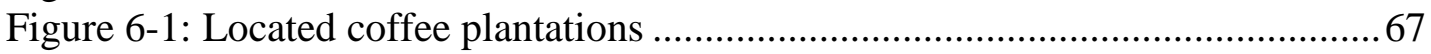

Figure 6-2: Potential coffee locations under current climate conditions ......................68

Figure 6-3: Potential coffee locations under climate increases ..................................69

Figure 6-4: Potential coffee locations under climate decreases ..................................70

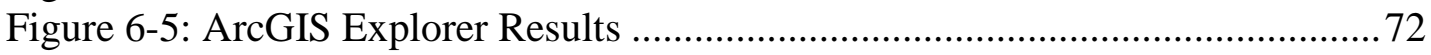

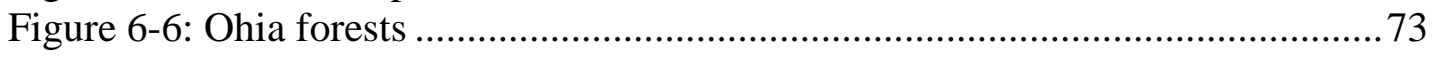

Figure 6-7: Potential ohia forest locations under current climate conditions .............74

Figure 6-8: Potential ohia forest locations under climate increases ...........................75

Figure 6-9: Potential ohia forest locations under climate decreases ............................76 
Figure 6-10: Ohia Forest Precipitation Attribute Distribution ............................... 77

Figure 6-11: Potential areas for each of the four attributes individually................... 78

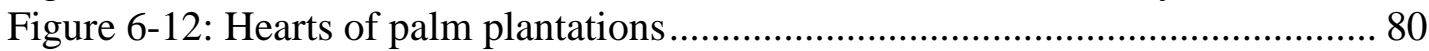

Figure 6-13: Potential hearts of palm locations under current climate conditions.... 81

Figure 6-14: Potential hearts of palm plantations under increases in climate........... 82 


\section{List of Tables}

Table 5-1: Summary statistics for each dataset and each vegetation type ....................... 48 



\section{List of Acronyms and Definitions}

$\begin{array}{ll}\text { DEM } & \text { Digital Elevation Model } \\ \text { ESRI } & \text { Environmental Systems Research Institute } \\ \text { GIS } & \text { Geographic Information Systems } \\ \text { IDW } & \text { Inverse Distance Weighted } \\ \text { LiDAR } & \text { Light Detection And Ranging } \\ \text { NAD } & \text { North American Datum } \\ \text { NOAA } & \text { National Oceanic and Atmospheric Administration } \\ \text { NRCS } & \text { Natural Resource Conservation Service } \\ \text { RCC } & \text { Regional Climate Center } \\ \text { SQL } & \text { Structured Query Language } \\ \text { USGS } & \text { United States Geological Survey } \\ \text { UTM } & \text { Universal Transverse Mercator } \\ \text { VBA } & \text { Visual Basic for Applications } \\ \text { WGS } & \text { World Geodetic System } \\ \text { WRCC } & \text { Western Regional Climate Center }\end{array}$





\section{Chapter 1 - Introduction}

Temperatures, precipitation rates, and elevations are three of the factors that power the ecology/landscape on Hawaii. This project produced a solution to study how the Hawaiian vegetation changes when temperature and precipitation variables change, while factoring in effects of elevation. A vegetation model was developed to predict the impact of precipitation, temperature, and elevation on Hawaiian vegetation types. More specifically, individual agricultural and environmental vegetation types (such as coffee plantations, papaya plantations, koa forests, etc.) were analyzed. The Hawaiian Vegetation Model includes an interactive tool that allows county planners to understand the Hawaiian vegetation under increases and decreases in temperature and precipitation from their current averages. The established predictive vegetation model that determines specific types of vegetation may assist county planners.

\subsection{Client}

Dr. Karen Kemp, of the Kohala Center in Waimea, Hawaii, was the client this for this project. In addition to being the main point of contact, Dr. Kemp provided reference information about the island of Hawaii regarding the specific processes of the project, such as which variables are appropriate to develop an accurate vegetation model. Dr. Kemp was particularly interested in providing county planners with a tool to estimate where vegetation types would most likely be located if the average temperature and precipitation rates changed, while factoring in elevation. In addition to the vegetation types dataset Dr. Kemp provided for the project to complete this task, Dr. Kemp also suggested creating a new remotely sensed dataset in order to compare it to the provided remotely sensed dataset to ensure the placement accuracy of the vegetation types.

\subsection{Problem Statement}

The purpose of designing the GIS vegetation model was to understand how locations of vegetation change when temperature and precipitation factors vary from their averages, while also incorporating static elevation changes. GIS modeling was used as a solution to estimate these values on Hawaii, the study area for this project (Figure 1-1). Specific agricultural and environmental vegetation types (such as coffee plantations, koa forests, etc.) were dynamically mapped to show the effects varying temperature and precipitation rates, and static elevations have on the specified vegetation types. The lack of knowledge about future placement of vegetation cannot be predicted perfectly, thus making GIS modeling a useful tool. However, producing a vegetation model with the aid of GIS can provide the county planners with an educated best guess in determining how precipitation, temperature, and elevation affect the Hawaiian vegetation. 


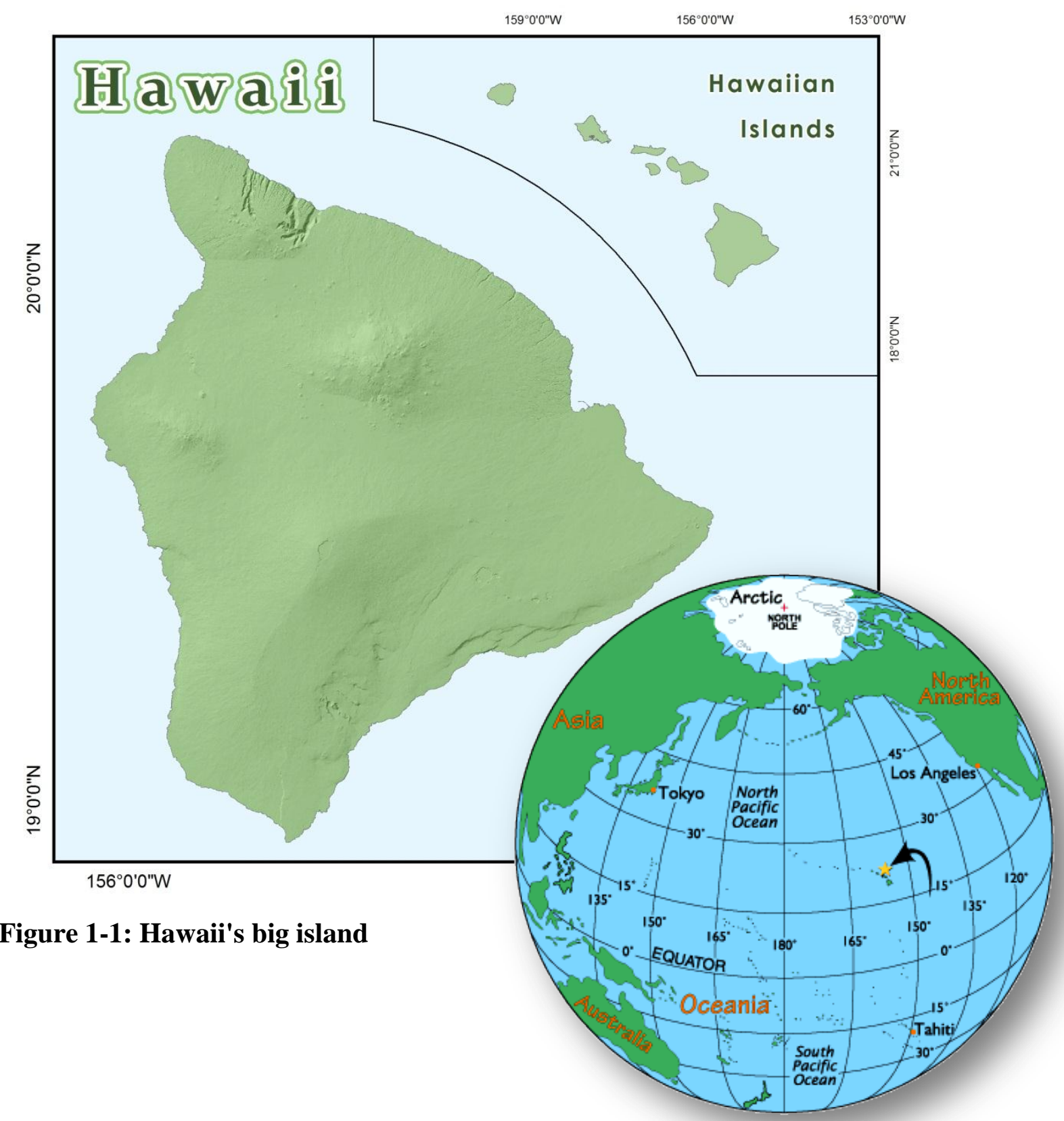




\subsection{Proposed Solution}

The intended solution of the Hawaiian Vegetation Model was to produce a user-friendly tool for county planners interested in performing landscape changes on Hawaii. Agricultural and environmental vegetation types, such as coffee plantations, banana plantations, and ohia forests, were some of the estimated crop and forest types used for this project. ESRI ArcGIS software package permitted the creation of the vegetation model. For the model (Appendix 1), the current temperature, precipitation and elevation averages were calculated for the individual vegetation types. When the user enters new temperature and precipitation estimates, the potential locations of each individual vegetation type changes according to what the user entered.

\subsubsection{Goals and Objectives}

The Hawaiian Vegetation Model was designed to predict climate change's impact on the Hawaiian landscape. Precipitation and temperature climatic variables were used to determine what effect climate has on the Hawaiian vegetation. For example, if average Hawaiian temperatures drop three degrees Fahrenheit over the next thirty years, it is likely that the potential locations of flora and fauna will shift; thus for example, the county planners in Hawaii could reclassify the Hawaiian landscape from farmland to pastureland and still be able to find use of that plot of land if agriculture is no longer viable. Conversely, perhaps climate change will be dramatic enough that coffee plantations will be rare, but still able to survive on Hawaii. County planners would then need to discover all of the potential areas where new coffee plantations could grow under the new environmental scenario.

\subsubsection{Scope}

The Hawaiian Vegetation Model was designed in ArcGIS 10's Model Builder application. The Model Builder application allows a string of geographic-processing functions to work together to complete an overall superior process. The creation of a scripting tool allows county planners to view and decipher decisions about agricultural development planning. A final database, which includes the data models, Python scripts, all associated data, and text help files serves as the deliverables for this project.

\subsubsection{Methods}

Creating the Hawaiian Vegetation Model was accomplished in a series of steps. As opposed to traditionally constructing the model in a raster format, this project attempted to explore if the results could be constructed in a vector format (Section 5.2). The first step was determining which variables to select from existing climate change models. Temperature and precipitation variables served as inputs for finding out how climate change affected the Hawaiian landscape. Furthermore, each climate variable holds an infinite amount of scenarios that could potentially happen. For example, the precipitation change inputs have a blank input for the user to specify a number to enter in ratio format. If the user enters the value 0.5 , then the user is halving the current average precipitation rates. If the user enters 1 , then the user is leaving the average precipitation rates as they 
currently are. Finally, if the user enters 2, then the user is doubling the current average precipitation rates. These instructions also appear on the script dialog box created for the vegetation model (Figure 1-2).

\title{
Enter Precipitation Ratio
}

\author{
Enter a numeric ratio to alter the average annual \\ precipitation. A value entered of "1" will reflect the \\ current precipitation, a value entered of "2" will \\ reflect doubling the current precipitation, and a \\ value entered of " 0.5 " will reflect halving the \\ current precipitation.
}

\section{Figure 1-2: Vegetation model instruction}

The second step was determining which average temperatures, precipitation rates, and elevations were associated with each agricultural and environmental vegetation type. For the vegetation types, a kriging algorithm created raster surfaces from point values to interpolate the temperature and precipitation datasets throughout Hawaii. Important to note is that the interpolated surfaces did not completely represent the island with temperature and precipitation, creating "no data" on the southern and eastern tips. This was because the eastern and southern most collected sample points were not located on the eastern and southern most extremes of the island (Section 5.2.1). Then, from a remotely sensed vegetation land cover dataset, each land cover type with a substantial amount of records was individually extracted and matched up with each individual climatic dataset to decipher the ranges of temperature, precipitation, and elevation that currently cover each individual vegetation variable (such as coffee plantations, koa forests, etc.).

\subsection{Audience}

The intended audiences of the vegetation model are county planners who are interested in agricultural and environmental vegetation variations and changes due to altering climatic factors of average temperatures and precipitation rates. It is expected that the county planners will have access to ESRI's ArcGIS software, in order to run the Python scripting dialog to produce the desired results. If not, the county planners can run the Python scripting dialog unrelated to ArcMap and view the resulting output feature classes in ArcGIS Explorer (free version). This paper assumes that the county planners will have at least basic familiarity with GIS. Furthermore, acronyms are used throughout the paper, but they are all defined in the acronym section if there is any confusion as to what a specific acronym is.

\subsection{Overview of the Rest of this Report}

The rest of the report explains the vegetation model project in full detail. Chapter two explains the research used to complete the project. This chapter outlines what a general data model and climate model are, in regards to this project. Chapter three reviews the 
project's system design and analysis. This chapter explains the project plan, what was delivered to the client, what technology is required for the project, and what software upgrades are needed. Chapter four examines the design of the project's database. This chapter reviews the data used for the project, where the data for the project came from, how the data was collected, and the data cleaning steps performed on the datasets. Chapter five discusses the project implementation phase. This chapter examines how the datasets were prepared to be entered into the data model, how the temperature, precipitation, and elevation ranges were determined for each vegetation type, what tools were used manipulate the datasets in the data model, and how the interpolated datasets were tested for accuracy. Chapter six discusses the results and analysis of the project. This chapter explains how to use the script dialog box included for the model, what the results could potentially be from a few sample vegetation type trials, and what went right and wrong throughout the duration of the project. Finally, chapter seven summarizes the entire project. This chapter reviews the project goals, how the goals were accomplished, what the project results were, and what future projects could result from the completed Hawaiian Vegetation Model. 



\section{Chapter 2 - Background and Literature Review}

\subsection{Introduction}

The main objective of the Hawaiian Vegetation Model was to create a model that predicts potential locations of specific vegetation types from various temperature and precipitation inputs. A climate change model (which can predict future temperature and precipitation) and a general data model (which can simulate differences of changing inputs) in terms of GIS first had to be understood in order to create a model that used similar approaches in each of the two researched models. The topic of a data model was researched in this paper, because a data model in the field of GIS is different from a data model in the field of business or engineering. Furthermore, section 2.2 addresses what a GIS data model consists of. A climate change model was researched because climatic data was used to predict potential vegetation change scenarios. Specific vegetation types thrive in specific climatic environments; therefore, section 2.3 explains why specific vegetation types have strict temperature and precipitation windows in which they can grow. Section 2.4 addresses temperature and precipitation variables as the most appropriate to use in climatic analysis. In addition, any gathered climatic data was in intervals of twenty to thirty year averages of data. Interpolation methods were required to create an estimated continuous surface of temperature or precipitation over the study area.

\subsection{The GIS Data Model}

One definition of a GIS data model is "a set of guidelines for the representation of the logical organization of the data in a database... [consisting] of named logical units of data and the relationships between them" (Tsichritzis \& Lochovsky, 1977, p. 21). A second definition is "a representation of the real world, based on specific inputs, while preserving a relationship between reality and the database" (Goodchild, 1992, p.401). These two sentences address a data model as a composite of the real world, represented digitally, by extracting data from a database. A GIS data model can also be conceptual while addressing the database design for maps in GIS format (Soller, Berg, \& Stamm, 2000). This GIS data model can either resemble a vector or raster data format (Goodchild, 1992); thus, the analysis under study will decide which data structure to use. For example, overlaying continuous surfaces is usually performed in a raster data format, while area calculations are usually performed in a vector data format. In either vector or raster format, however, a GIS data model provides organization of the spatial and attribute information associated with a map (Soller et al., 2000).

\subsection{Relationship Between Vegetation and Climate}

Very generally, the world is divided into climate zones that reflect the variations of temperature, precipitation, latitude, and elevation (Figure 2-1). For each variation, specific types of plants live in different fluctuations of temperature, precipitation, latitude, and elevation (Strahler \& Strahler, 1984). For example, a cactus would be 
common in areas with low precipitation, and tundra would be common in areas with high latitudes.
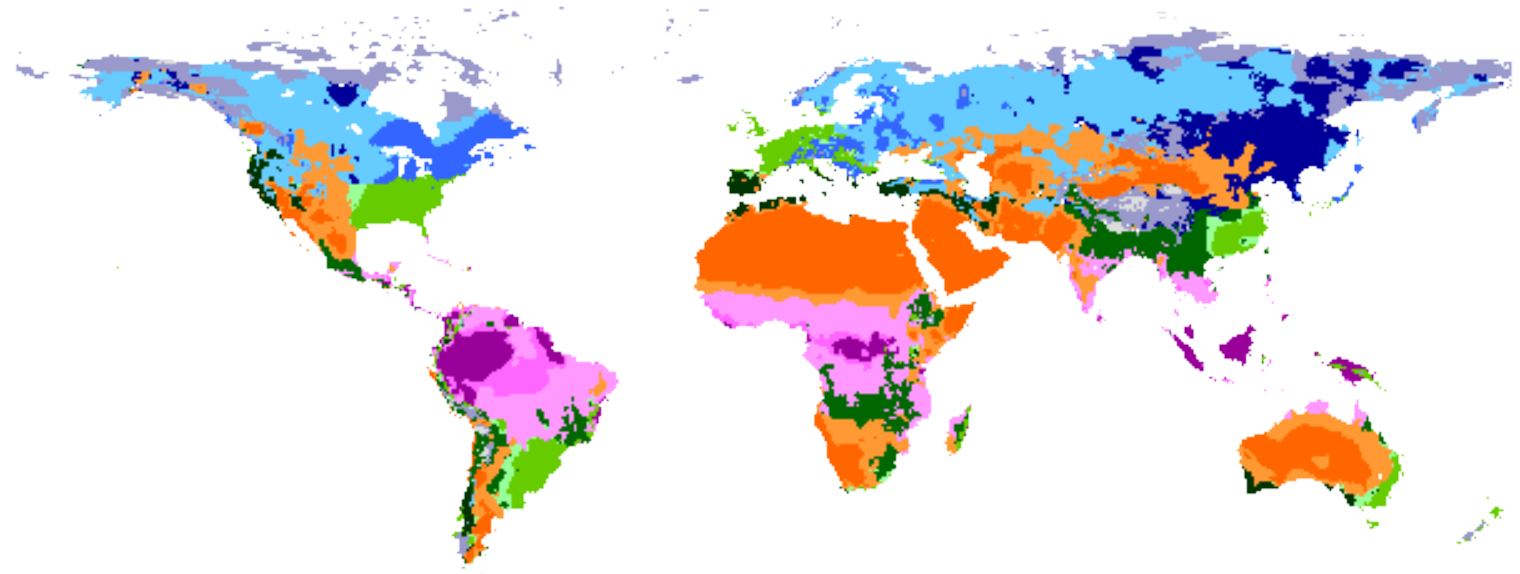

$\begin{array}{lcccccc}\text { Koeppen's Climate Classification } & \text { A } & \text { B } & \text { C } & \text { D } & \text { E } \\ \text { by FAO-SDFN-Agrometeorology Group - } 1997 & \text { Tropical } & \text { Dry } & \text { Temperate } & \text { Cold } & \text { Polar }\end{array}$

Figure 2-1: World climates. Source: $\underline{\text { ww.blueplanetbiomes.org }}$ 
Hawaii has varying differences in temperature, precipitation, and elevation. Any vegetation on the island will not vary significantly due to latitudinal changes (twenty degrees, sixteen minutes north as the northern boundary, eighteen degrees, fifty-four minutes north as the southern boundary) (Figure 2-2). However, if the average temperatures or precipitation rates around the island would change, then some of the potential locations of the individual vegetation types (coffee, avocados, koa forest) would potentially shift or disappear.

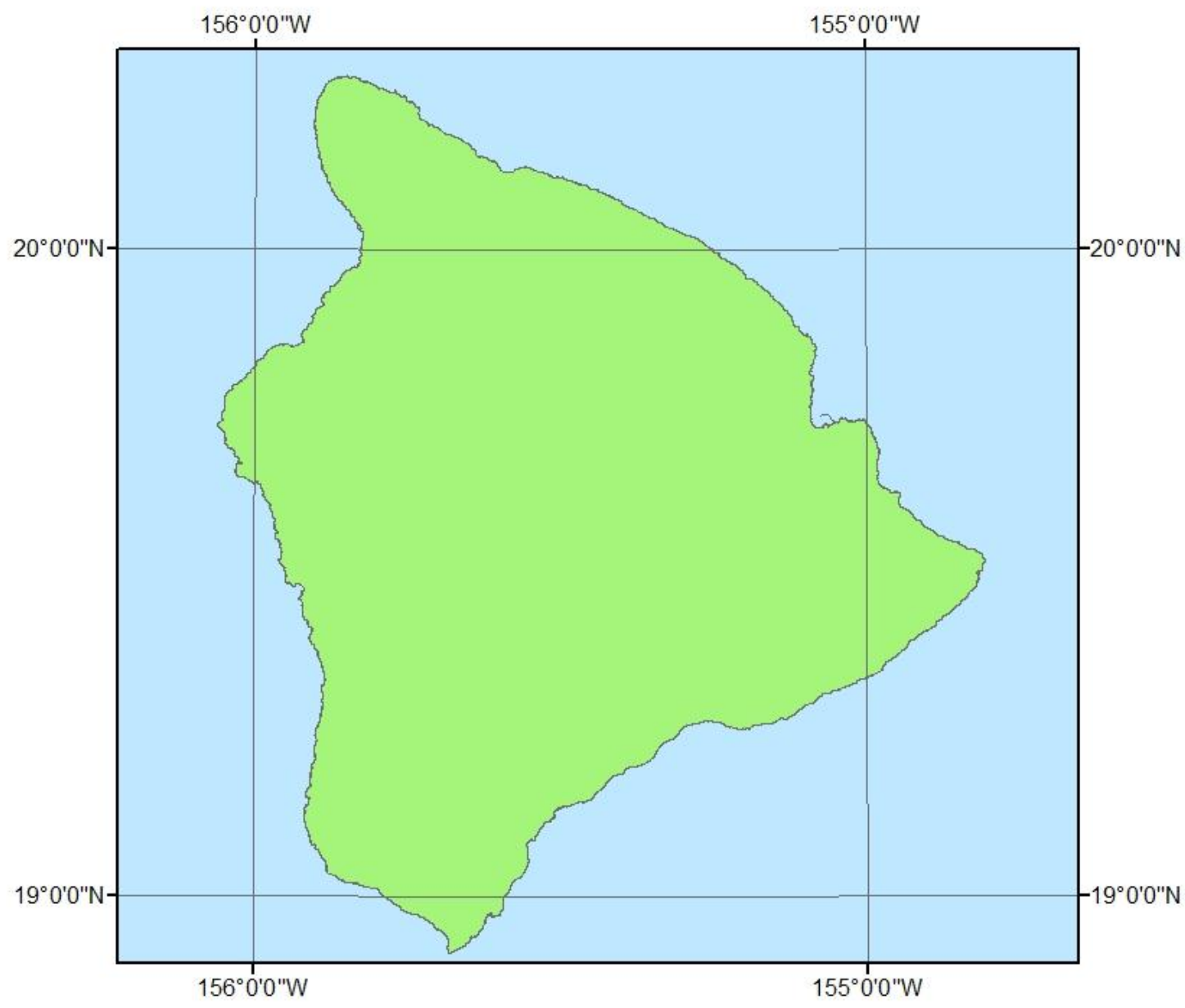

Figure 2-2: Hawaii's global position in terms of latitude and longitude

Looking at the average precipitation rates around the island, the general pattern has higher precipitation rates on the eastern side of the island and lower precipitation rates on the western side (Austin, 2002) (Figure 2-3). Therefore, any plants that thrive in drier climates will most likely thrive on the western side of the island, and any plants that 
thrive in wetter climates will thrive on the eastern side. Of course, some inconsistencies could be found on the drier side, since irrigation is used for certain crops.

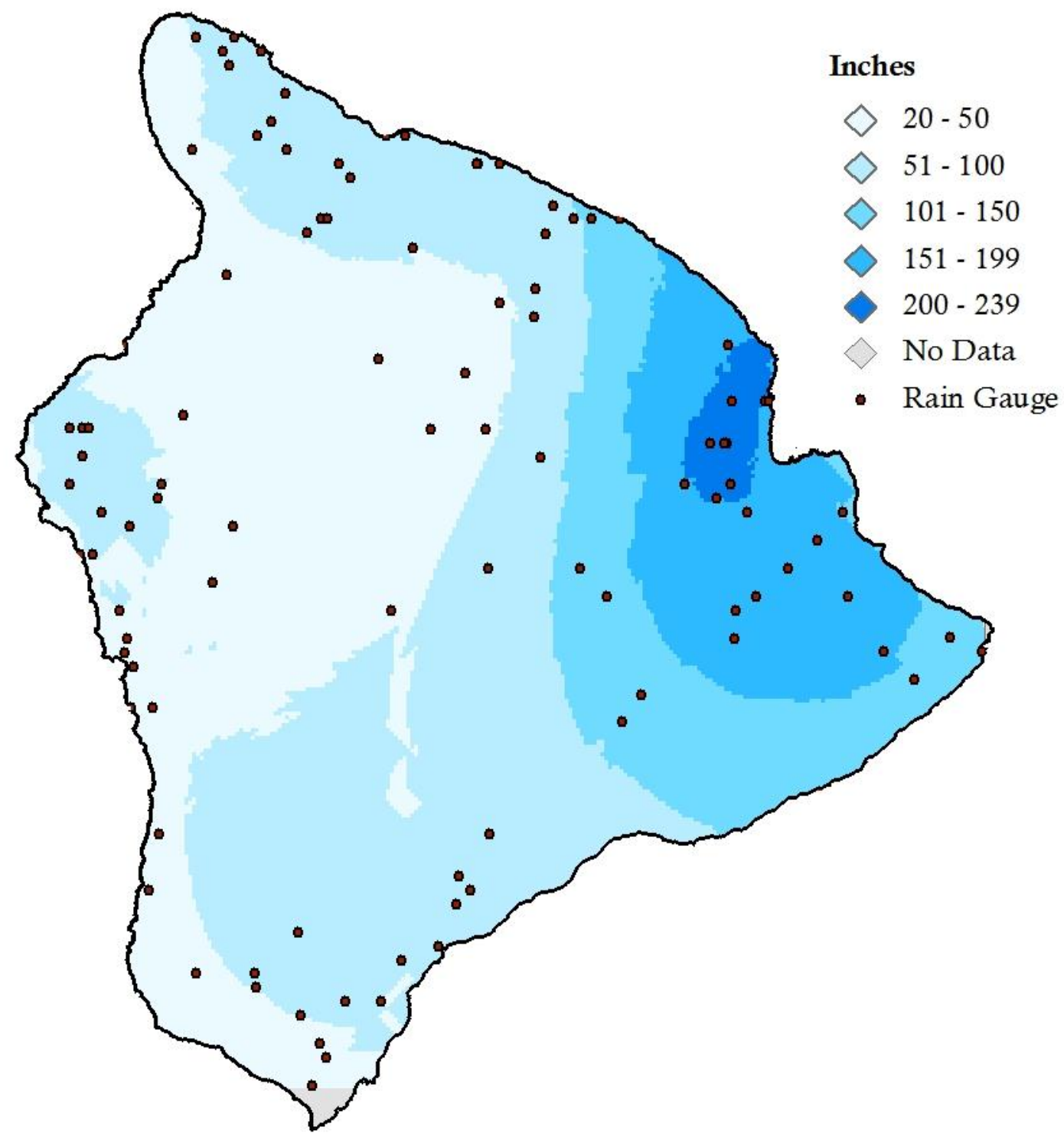

Figure 2-3: Hawaii's Annual Precipitation Averages

Looking at the average temperature rates around the island, the general pattern has higher temperatures around the coastal areas and lower temperatures in the inland areas (Western Regional Climate Center, 2006) (Figure 2-4). This appears to be a direct result of the elevation changes as five volcanic peaks make up the interior of this island (Figure 2-5). Therefore, vegetation has a correlation with temperatures and elevations on the island, where plants that thrive in cooler temperatures are found at higher elevations, and plants that thrive in warmer temperatures are found at lower elevations. 

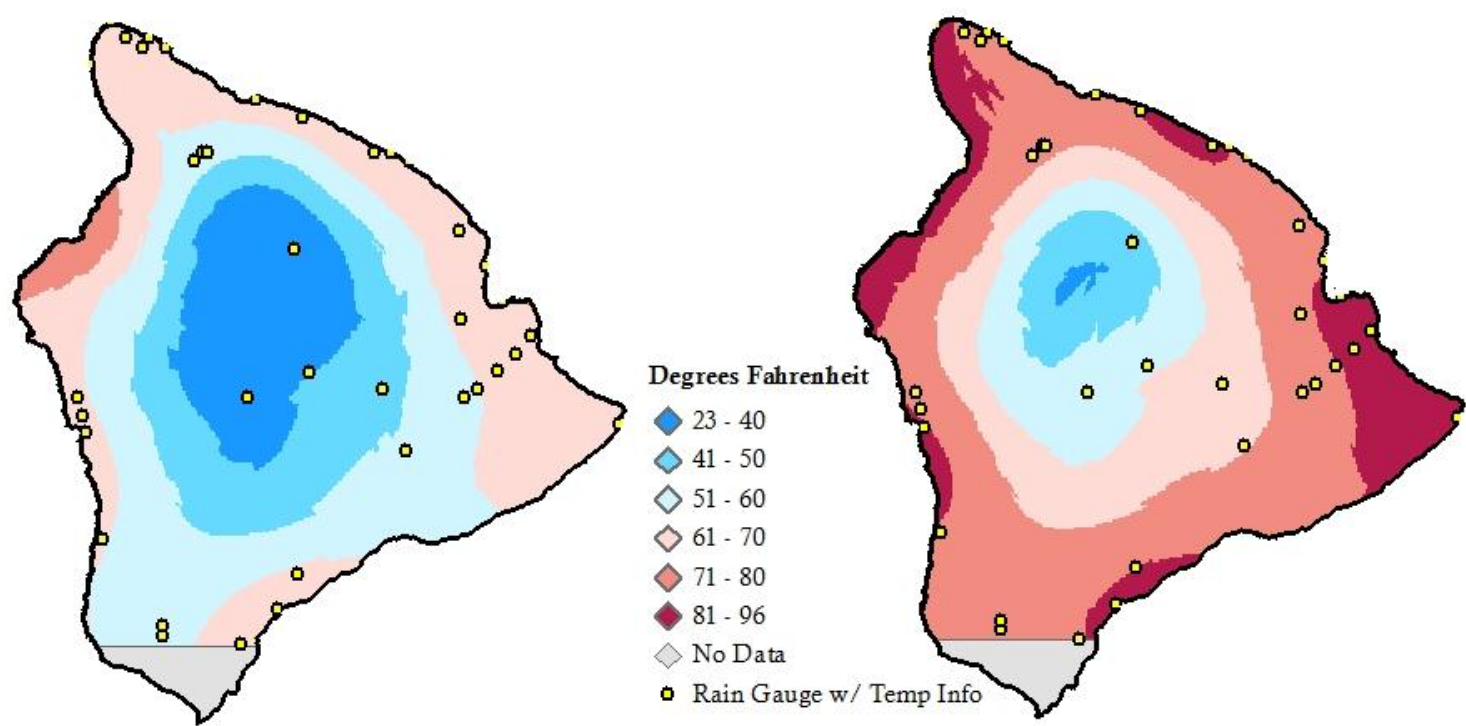

Figure 2-4: Hawaiian Minimum (left) and Maximum (right) Temperature

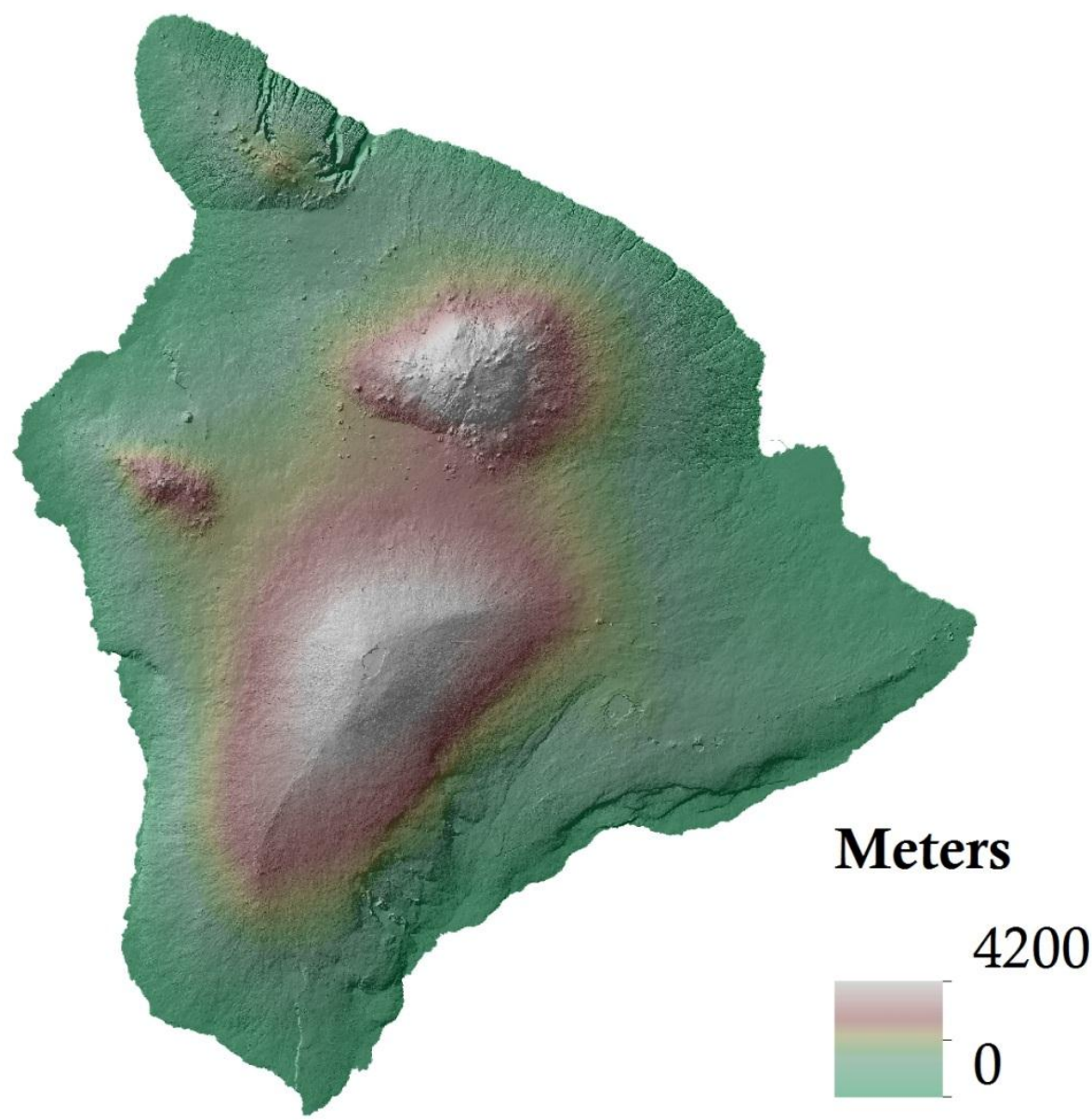

Figure 2-5: Hawaiian Elevation 


\subsection{The Climate Change Model}

A GIS data model can facilitate a wide variety of analyses. In this project, the climate changes represent a prediction of the precipitation and temperature scenarios on Hawaii. In a study by Ninyerola, Pons, and Roure, they predicted air temperature and precipitation in Northeast Spain for their climatological analysis (2000). Scientists collected data from meteorological stations that were geographically set apart from each other approximately every 125 kilometers for precipitation data and approximately 200 kilometers for temperature data. Values between meteorological stations were determined by interpolation rather than by drawing isolines (Ninyerola et al., 2000). Climate data intervals usually represent a thirty-year average of the variable under study (Bell \& Walker, 2005). However, this study specifically mentions the use of precipitation data in intervals of 20 years and air temperature data in intervals of 15 years (Ninyerola et al., 2000).

In a different climate change model, the use of the term "limited area model" simulates the regional distribution of precipitation and surface air temperature in the continental United States (Giorgi, Brodeur, \& Bates, 1993). Precipitation and surface air temperature are the primary focus because these two are usually of importance for any climate change impacts. Researchers used a method of downscaling in the study to avoid the basic seasonal and regional climate characteristics that large-scale data produces in prediction scenarios. For comparative measures, present-day surface temperatures and precipitation rates were recorded, as well as a prediction of these rates $3 \frac{1 / 2}{2}$ years into the future with doubled carbon dioxide levels. Finally, the researchers compared these two scenarios using spatial statistics such as averages, spatial standard deviations, and correlations (Giorgi et al., 1993).

\subsection{Summary}

For this project, it was necessary to grasp the general concepts of a basic GIS data model and a climate change model in order to understand how to incorporate similar processes from these models into the Hawaiian Vegetation Model. GIS data models in general are representations of vector and raster data formats. This project used raster data for data analysis as well as vector data to complete the geoprocessing tasks in ArcGIS's Model Builder application.

A strong connection exists between the type of climate (temperature and precipitation) and the types of vegetation that exist in an area. Section 2.3 explained how changes in temperature and precipitation could cause a shift in the overall vegetation patterns. This project used a dataset derived from a remotely sensed vegetation analysis raster layer as well as interpolated temperature and precipitation surfaces, and a DEM to estimate the temperature, precipitation, and elevation range for each vegetation type (coffee, papaya, koa forest, etc.).

A climate change model usually represents precipitation and temperature scenarios to perform predictive analysis, as shown in section 2.4. In addition, a climate project should ideally average any gathered temperature or precipitation data over a period of 20 to 30 years to improve accuracy of the estimates. After the data were collected, proper 
interpolation techniques should be used to estimate temperature and precipitation averages for areas where no data has been collected. The project collected 41 sample points for average temperatures around Hawaii (Appendix 2), and 115 sample points for precipitation averages (Appendix 3 ). Temperature and precipitation data are yearly summaries over the period of 1971 to 2000 . Kriging was used to interpolate any areas with unknown climatic values. 



\section{Chapter 3 - Systems Analysis and Design}

\subsection{Introduction}

A system analysis and design of the Hawaiian Vegetation Model was accomplished through the creation and completion of a requirements analysis, a system design, and a project plan. The requirements of the project were broken up into functional and nonfunctional requirements, found in section 3.3. The functional requirements allow the user to select an area of interest, predict the future temperature and precipitation ranges on Hawaii, and observe the differences in the vegetation changes from the current vegetation (section 3.3.1). The non-functional requirements specify the technology needed to run the model, the updates needed to sustain the functionality, and information needed to transfer the model from worker to client (section 3.3.2). The technology needed included installing ArcGIS onto a personal computer. The updates included installing various software upgrades and plug-ins. The information needed for transfer required creating a database in ArcMap that held the script, model, and data, as well as the help file that explains how to use the vegetation model.

The system design includes a vector-based geographic processing model to execute the geographic processing functions of the vegetation model. The geographic processing model creates the result that displays on the map. The user has the option to choose various temperature degree changes and precipitation ratios in the model to make an educated guess as to where potential locations of the vegetation types will exist. The created script dialog box was intended to provide the users with a simplistic approach of understanding the impact climate change has on the vegetative landscape, rather than a more complex approach.

In section 3.5, the strategic goal of this project included creating an efficient model that could predict potential vegetation locations from various temperature and precipitation inputs. A problem that the project plan addressed was establishing the best way to communicate the predictive vegetation results to the intended audience of county planners. The delivered project included a database containing the model, the data associated with the model, the script and dialog box used to run the model, and the associated help files. Finally, the project's ultimate success depends on whether or not county planners find the vegetation model useful.

\subsection{Problem Statement}

The Hawaiian Vegetation Model uses predictive temperature and precipitation averages and static elevations to estimate the potential areas where specific vegetation types could occur. In order to perform a geographic analysis of this problem, GIS modeling was the solution. This model was useful because in any predictive analysis, the outcomes cannot be certain; rather, the best prediction that a person can make about the future is the location where an event might occur. Using the Hawaiian Vegetation Model, county planners can make educated guesses as to what the future temperature and precipitation averages will be in order to determine a potential location for a specific vegetation type. 


\subsection{Requirements Analysis}

\subsubsection{Functional Requirements}

Upon completion, the functional requirements of the Hawaiian Vegetation Model will allow the user to identify an area of interest, input their own climate variables, observe how the landscape changes based on the climatic inputs, and compare these changes to the original landscape for the vegetation model portion of the project. County planners will operate this model through ArcMap or ArcGIS Explorer. The model will calculate temperature and precipitation averages entered by county planners and process them into potential zones for the specific vegetation type to flourish, based on the changes in climate as entered by the user. The model's scripting dialog will specify an output location for data exchange capabilities before running the model's scripting dialog, to allow the county planners to do comparative analyses between other climatic outputs on the Hawaiian landscape. For example, Figure 3-1 compares how the best potential locations of the ohia forest vegetation type would change if precipitation rates on the island increased by 20 percent and 80 percent.

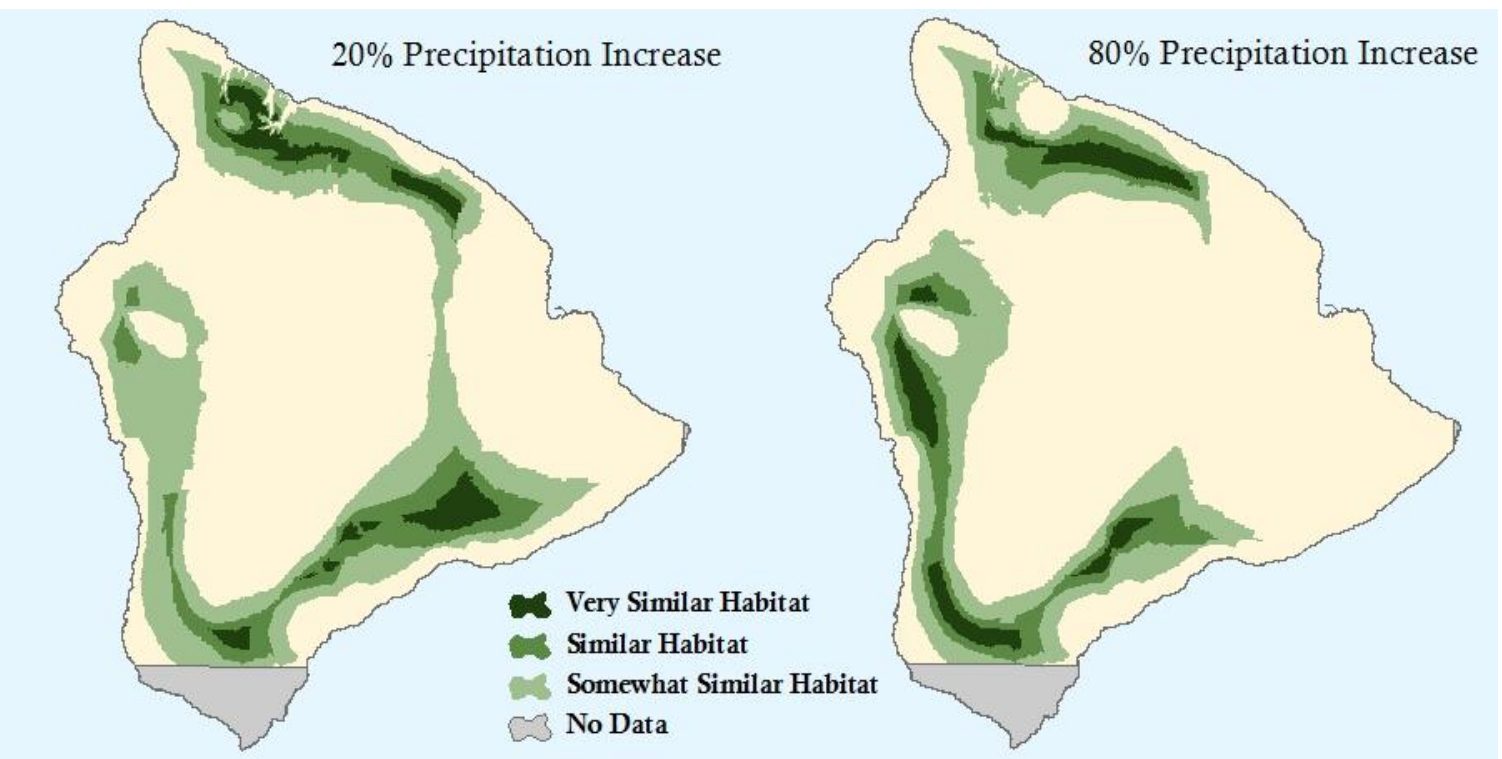

Figure 3-1: Ohia forest comparison

\subsubsection{Non-Functional Requirements}

The non-functional requirements of the Hawaiian Vegetation Model specify the technology needed to run the model, the updates needed to sustain functionality, and information needed to transfer the model from worker to client. The technology needed to run the Hawaiian Vegetation Model from a personal computer will include either purchasing ESRI's ArcInfo/ArcEditor/ArcView or downloading ESRI's ArcGIS Explorer and running the associated Python script (Appendix 4, Appendix 5). ArcGIS was used for various geoprocessing tasks for creating the model, but the user does not need to 
purchase this software to run the model, as this project intends to provide county planners with spatial analysis without the need to purchase an ESRI software package. If the user does not intend to buy an ESRI software package, the user will need to have an internet connection to download ESRI's ArcGIS Explorer application in order to view any outputs generated by the Python scripts.

Over time, if the Hawaiian Vegetation Model is deemed useful to county planners, various ArcGIS and Python software upgrades may need to be downloaded in order to maintain the functionality of the model. In addition, the installment of plug-ins to the user's personal computer may be necessary for any ArcGIS and Python software to run smoothly. The generated models will use various temperature and precipitation averages, and static elevations to determine potential vegetation areas through a series of hands-free or automated processes. To ensure proper functionality, a series of trials were ran in the ArcGIS Model Builder application. Furthermore, the maintenance required for the models to run smoothly involves installing the necessary plug-ins and updates for the user's personal computer as well as any ArcGIS and Python software updates or plug-ins. If the Hawaiian Vegetation Model is useful to county planners, then the client could archive the model for permanent use. Otherwise, the client will use the model as a prototype for a more detailed vegetation model.

The vegetation model was tested to ensure that the model ran smoothly in Model Builder, and the script ran smoothly in ArcGIS and Python, without any errors. The training needed to run this project is oriented from a county planner's standpoint. The planners have to be aware of the impacts of climate change on vegetation types to extract information from the model or script. In order to deploy the model or script, not much preparation is required. The model and script are each placed inside the database; simply opening either the model or script allows the user to perform predictive climatic analysis on the vegetation types by entering a temperature change and a precipitation ratio in the associated scripting dialog box.

Of the above requirements, the functional requirements were the most challenging to complete because the majority of the project time was spent associating the data with the tool functions, so users can accurately analyze the Hawaiian landscape. This modeling application is intended to aid county planner's research by finding an easy way to provide a spatial analysis tool to a secondary audience. The largest obstacles were implementing user inputs into a model or script. This required writing code in Python and linking the written code back into an ArcGIS tool.

\subsection{System Design}

The system for the Hawaiian Vegetation Model was designed for ESRI's ArcGIS software. An additional Python script was included to produce results in ArcGIS Explorer, in case county planners are unable to purchase ESRI software. Once any of the software packages are installed on a personal computer, the outcomes of the Hawaiian Vegetation Model will be visible through a series of feature classes. The model was developed in ESRI's ArcGIS software, which is why the user of the model must at least download and install ESRI's ArcGIS Explorer to run the processes. The user's initial understanding of how to run the Hawaiian Vegetation Model is also important. Instructions provided in the Python script dialog box or in the text help file on how to run 
the vegetation model and what the outputs represent for either ArcGIS or ArcGIS Explorer will help the users understand the vegetation model and its associated outputs.

The Hawaiian Vegetation Model's final result is an assortment of exported feature classes, as opposed to a digital interface or static maps. The exported feature classes represent the various temperature and precipitation estimates performed on the specific vegetation types. When used, new feature classes are created after the user enters different climatic inputs, comparing one climatic output against another to understand the different potential vegetative locations. For example, the user opens the vegetation scripting dialog, then enters the desired minimum temperature degree change, the maximum temperature degree change, and the desired precipitation ratio. The user then chooses a vegetation and habitat type to predict the potential location, based on the previously entered values for temperature and precipitation. Finally, the user exports the new feature class to a database and can compare the potential locations of the exported feature class to the potential locations of another exported feature class or to the actual location of the specified vegetation type. Figure 3-2 shows a mockup of how the user might view the feature class output after a model run. After the script is executed, the new potential locations of the vegetation type can be overlaid in ArcMap.
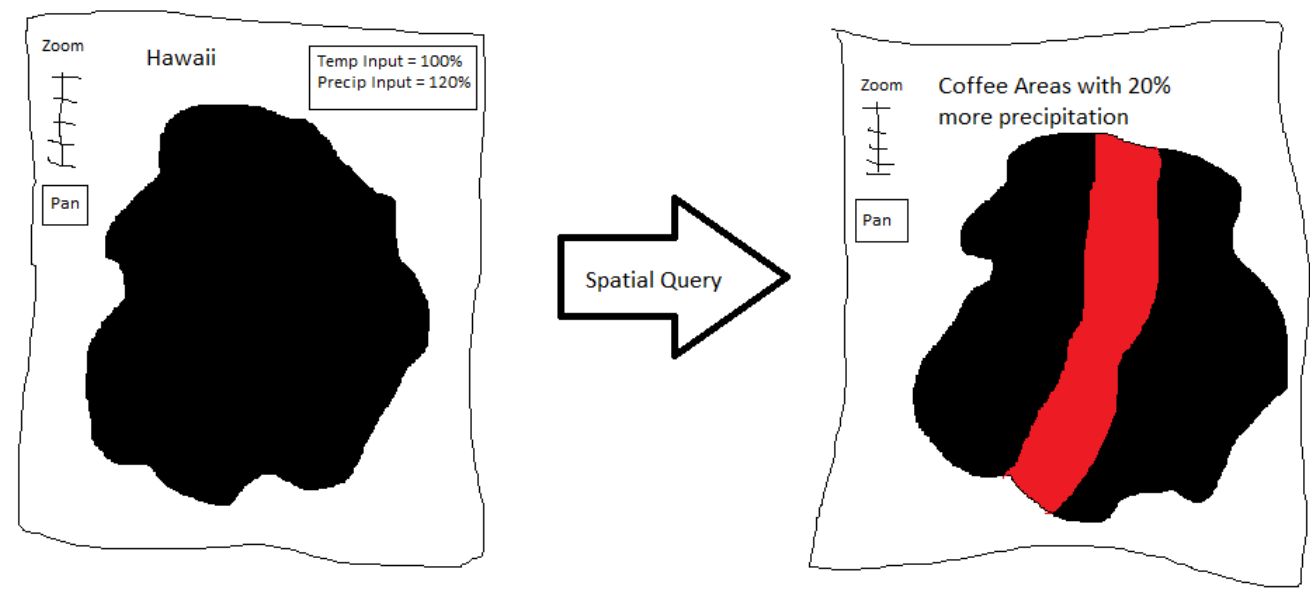

Figure 3-2: Vegetation map and technology mockup

The user runs through a series of steps for each phase of the model in order to produce a set of vegetation outputs. From a design perspective, one accomplishment was to make the scripting dialog and its functions as simple as possible, rather than incorporate more decision options into the scripting dialog of the model. A user should be able to sit down at a personal computer, read the associated directions, and understand how to produce a specified output, rather than having the user experiment with the scripting dialog before being able to understand how to produce an output. The purpose of the resulting map is to show county planners how vegetation types can change with changes in the environment on Hawaii. The scripting dialog would become too complex and fade away from its purpose if additional options in the scripting dialog allowed the user to manipulate the potential location of the vegetation types around urban areas or 
government owned land. This has the potential for confusion as to why there would be large abrupt data gaps in the output map.

\subsection{Project Plan}

The Hawaiian Vegetation Model uses a modeling process to communicate information to an audience seeking to save time without performing the analysis themselves. It had one strategic goal to create a model that shows how the vegetation changes, given specific temperature and precipitation changes from their current averages. This goal helped to accomplish the client's need to provide county planners with a tool to determine how the vegetation locations would change as the climates changed.

The problem that the Hawaiian Vegetation Model addressed included determining what the Hawaiian landscape will look like in the future. In the sense that a changing climate could potentially alter vegetation patterns on Hawaii, the solution was to predict how the vegetation could change using specified temperature and precipitation inputs. A prediction model was created in which various temperature and precipitation scenarios served as the inputs of how the vegetation changed. For example, using the current average precipitation rates on Hawaii, the climatic precipitation rates might increase 20 percent from the current averages, while the minimum and maximum temperature rates might increase by two-degrees Fahrenheit from their normal averages. This would produce different potential locations of any vegetative type.

The Hawaiian Vegetation Model's project plan was broken down into three phases: collection of appropriate climate data for the variables of temperature and precipitation on Hawaii; modeling the climatic data in a vector format to show what effect temperature and precipitation have on the vegetation; and comparing the altered vegetation to the present-day vegetation. The collection of appropriate data rested upon variables that previous research articles had used. For example, temperature and precipitation data were the most common among predicting climates (section 2.4) and thus, used for this project. Modeling relied on discovering what climate and elevation conditions specific vegetation types currently exist under and then using the current conditions to predict the potential locations under a change in the current climates for the model. Finally, the users would ideally compare the results generated from the altered climate scenarios to the present-day locations of the vegetation types to observe the changes that differing climates produce.

Of the included deliverables, a Python scripting dialog allows the user to input a maximum and minimum temperature degree change, a precipitation ratio estimate, a vegetation type through a selection box, a habitat type through a selection box, and a location to store the output file. The data to run this model/script is included in the database, which stores the following feature classes: current vegetation types, the boundaries of Hawaii, areas with insufficient results (no data zones) (section 1.3.3), and a polygon layer that aggregates current elevation, temperature, and precipitation attributes.

Other deliverables for this project are text files that explain how to use the scripting dialog and associated model. Two sets of instructions are available. One set explains how to run the scripting dialog and what the outputs represent in ArcMap. The other set explains how to run the scripting dialog in Python and view the feature class results in ArcGIS Explorer. Details about how to install and run ArcGIS Explorer and Python were included to ensure that the county planners have the proper software to 
execute the modeling applications. Ultimately, if the county planners find this project useful and easy to use, then this project will be a success.

\subsection{Summary}

The completion of the system analysis and design of the Hawaiian Vegetation Model project required an extensive requirements analysis plan, a system design plan, and a project plan. Functional and non-functional requirements in sections 3.2.1 and 3.2.2 respectively, comprised the requirements analysis plan. The functional requirements established that the users would predict their own temperature changes and precipitation ratios to calculate the differences in the vegetation types on Hawaii. The non-functional requirements covered the more technical aspects of the project, which included specifying the technology and updates needed to run and maintain the vegetation model, and project delivery. The technology and updates needed to run the model are ESRI's ArcGIS software and its upgrades and plug-ins. A database was delivered that contains the model, the script used to run the model, the data that the model references and the associated help files.

A detailed plan of the geoprocessing model and the associated data of the geoprocessing model made up the system design aspect of the project in section 3.4. The output feature classes that display on the map are the results of the geoprocessing model (executed scripting dialog). In the geoprocessing model, the user makes educated guesses as to what the future temperature and precipitation averages will be in order to manipulate individual vegetation types. The data referenced by the geoprocessing model was a feature class containing the elevation, average minimum and maximum temperatures, average precipitation rates.

Finally, the established project plan in section 3.5 met an overall strategic goal, addressed potential problems, and decided what to deliver to the client. The overall strategic goal of the project involved creating a modeling process that could determine various individual potential vegetation locations from numerous temperature and precipitation inputs. The deliverables sent to the client consisted of a single database that contains the model, Python scripts, the data that the model referenced to perform analysis, and the associated text help files that show the users how to run the model and what the results will be. The ultimate success of the project will be determined by how useful county planners find the modeling application to be. 


\section{Chapter 4 - Database Design}

\subsection{Introduction}

The Hawaiian Vegetation Models' database design includes elevation, temperature, precipitation rates, and vegetation types in the data model. The database includes an output feature class that the model edits each time the model runs, a data model, and a script with associated dialog box instructing how to run the vegetation model. The data came in shape file format for vegetation types dataset, a GRID raster format for the elevation dataset, and as text files for the temperature and precipitation datasets. Data scrubbing and loading was necessary for all datasets, but extremely necessary for the temperature and precipitation datasets for them to display properly in ArcMap.

The Hawaiian Vegetation Model used temperature, precipitation, and elevation attributes to discover the potential locations of specific crop and forest types on Hawaii. The attributes of temperature and precipitation are manipulated to simulate climate change and to predict the possible locations of the vegetation types. Elevation is a static variable, and cannot be manipulated because elevation heights hardly (if ever) change on a yearly basis.

A feature class, a model, and a script located in the file geodatabase made up the logical design of this project. The vegetation model used an initial feature class to perform predictive landscape analysis, which included temperatures, precipitation rates, elevations, and vegetation types. In addition, the feature class had mirror fields to store the predictive results, so the users could compare these predictive results to the original results. The model constructed in Model Builder displayed the steps the model took in order to create the predictive analysis, and was capable of running the model, as well. A script with associated dialog box was created for the model in order to provide users of the model with instructions for what temperature degree changes and precipitation ratios to enter. As an added bonus, the creation of the script also reduced the overall processing time it took to create the predictive output feature classes.

The vegetation land use dataset was created, designed, and updated by the National Resource Conservation Service (NRCS) located in the Hilo, Waimea and Kealakekua field offices on Hawaii. The elevation dataset was a digital elevation model (DEM) distributed by the Coastal Geology Group, which is a part of the University of Hawaii at Manoa. The Western Regional Climate Center (WRCC) had the necessary temperature and precipitation datasets. The WRCC collects its data from an assortment of agencies, including the National Weather Service and the National Oceanic and Atmospheric Administration (NOAA).

Data collection for the project included searching websites as opposed to the client providing all of the data. The vegetation dataset was obtained from the Redlands Institute in shape file format. The DEM came from the Coastal Geology Group's website in a GRID raster format. The temperature and precipitation datasets were transformed into text files from the WRCC website.

Data scrubbing and loading were required for each of the datasets gathered for the Hawaiian Vegetation Model. Specific crop and forest types were later extracted from the vegetation dataset in order to compare these individual attributes to the temperature, precipitation, and elevation ranges. Since the DEM came in a raster format, it was 
converted into a vector format to merge the layer spatially with the temperature and precipitation datasets later on in the project. The temperature and precipitation datasets required the most data scrubbing and loading. These datasets were opened in Microsoft Excel in order to merge and clean up each rain gauge text file into a single file to remove any unnecessary attributes. These files and related rain gauge files were then joined to create a shape file. The datasets were imported into ArcMap, so the spatial attributes could be drawn and exported to a feature class. 


\subsection{Conceptual Data Model}

The Hawaiian Vegetation Model included the classes of temperature, precipitation, elevation, crop types, forest types, and habitat types in its conceptual design. The purpose of the vegetation model was to discover how the potential locations of specific vegetation types could change when the average temperature and precipitation rates change.

The vegetation model includes the classes of elevation, temperature, and precipitation. Elevation was chosen because heights on Hawaii can change by hundreds of feet laterally in just a few feet horizontally (Figure 4-1). This is important because specific crop or forest types may only flourish in specific elevation ranges. The design used the classes of temperature and precipitation because these two variables are common among predictive climate analysis (Ninyerola, Pons, and Roure, 2000). These attributes determined which ranges would bound the environmental conditions favorable to a specific crop and forest types. For example, the geographic locations of coffee crops were compared against Hawaii's elevation, annual temperatures, and annual precipitation rates individually to determine the best potential climate and elevation ranges that coffee prospers in. The same process was completed for each of the crop and forest types until a unique range of temperature, precipitation, and elevation for each was determined for use in the prediction analysis. 


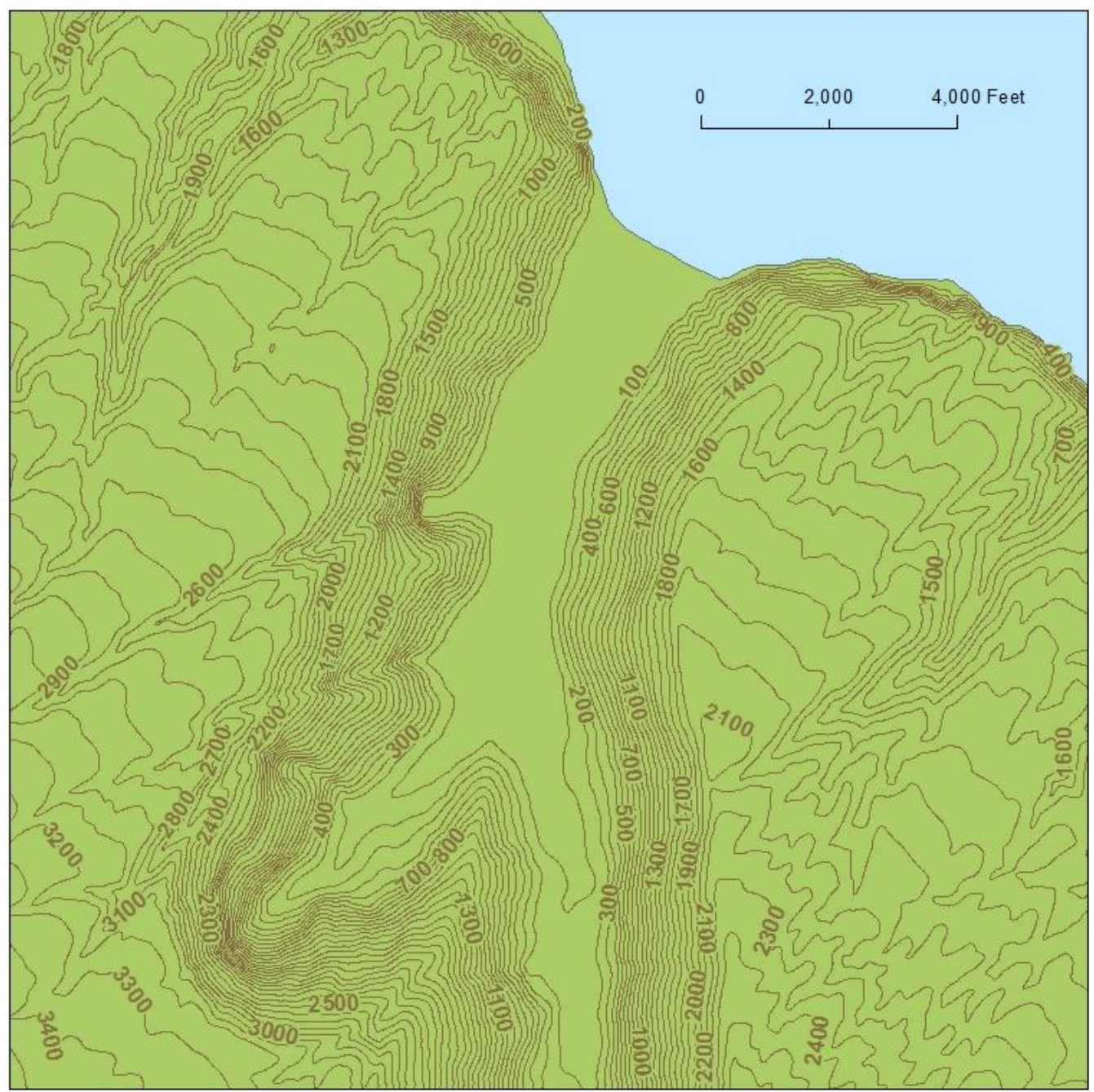

Figure 4-1: Hawaii's northeast coast. Source: Hawaii Statewide GIS Program

\subsection{Logical Data Model}

The Hawaiian Vegetation Model's logical model is comprised of a feature class, a model, and a script that runs the model. The vegetation model was constructed in a vector data format. The model produces an output feature class, in which features are edited to display different results, based on the user's input. The model displays how the processes edit the feature class when the user enters a specified input. The script's dialog box that runs the model displays a much more user-friendly dialog than the Model Builder interface for editing the feature class associated with the model. In addition, the script reduces processing time, and includes instructions for what numbers to enter, what the numbers mean, and what the results of the model will be in the dialog box. 
The model uses a vector data structure. A vector format was used because each feature in a vector file can hold multiple attributes as opposed to a single attribute held for each cell in a raster. Therefore, simple SQL expressions using AND can select from the aggregated dataset of minimum temperature, maximum temperature, precipitation, and elevation, each polygon that fell within the specified data ranges of all four attributes (Figure 4-2). For example, if looking at the vegetation type of coffee, it has the data ranges of 61 to 63 for minimum temperature, 76 to 80 for maximum temperature, 46 to 54 for precipitation, and 1125 to 1919 for elevation. All of the polygons with minimum temperature attributes between 61 and 63 would first be selected from the aggregate dataset (Figure 4-2, top left). Any polygons with maximum temperature attributes between 76 and 80 would be selected from the previously selected minimum temperature polygons (Figure 4-2, top right). Any polygons with precipitation attributes between 46 and 55 would be selected from the previously selected maximum temperature polygons (Figure 4-2, bottom left). Finally, any polygons with elevation attributes between 1125 and 1919 would be selected from the previously selected precipitation polygons (Figure $4-2$, bottom right). The model then simulates a temperature or precipitation change by updating the attributes of each polygon in the aggregate dataset, and then select new polygon areas using the data ranges for each vegetation type. 

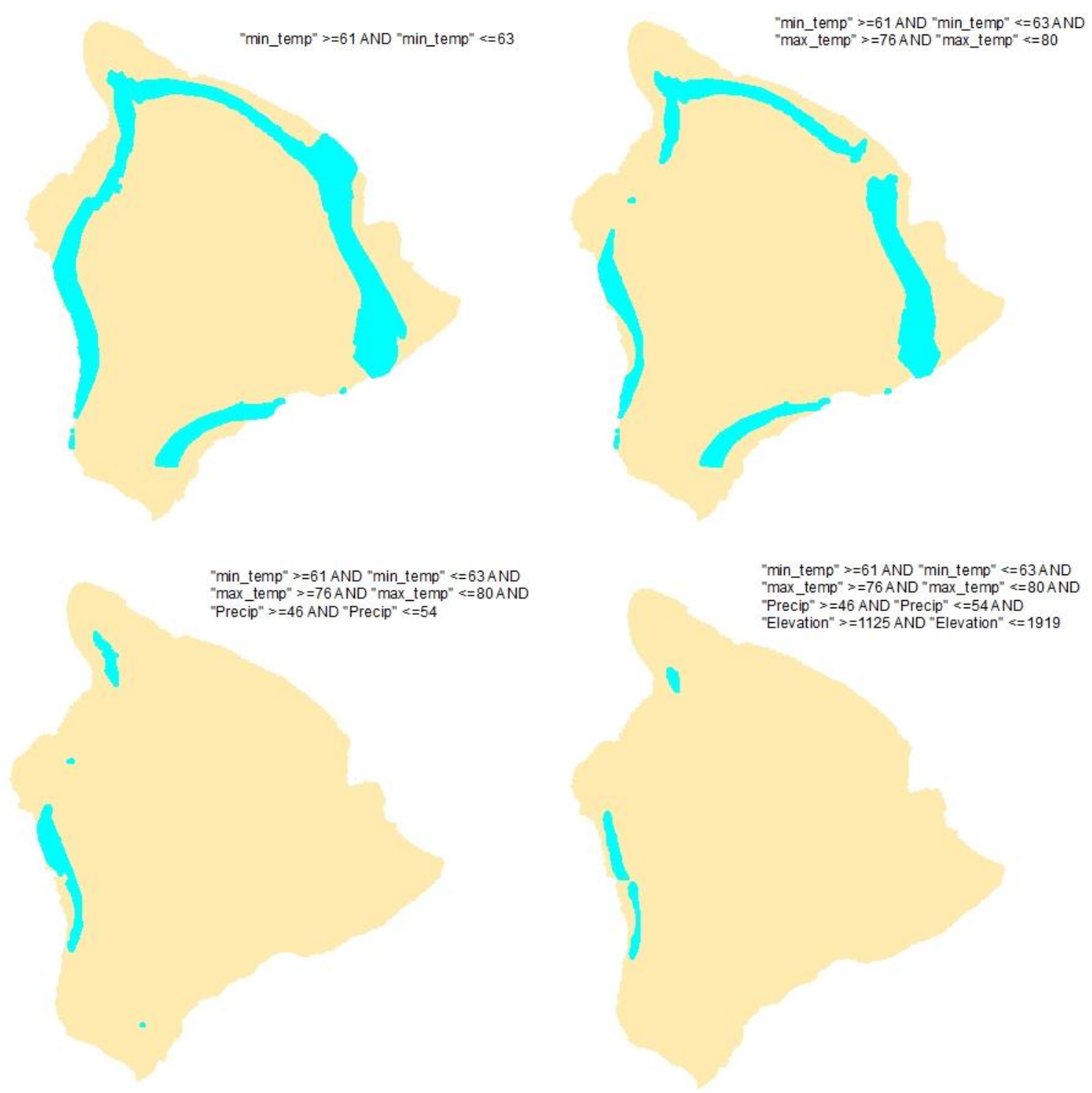

Figure 4-2: Attribute Specification of Vegetation Type

If this sort of analysis was performed in a raster data format as opposed to vector, over 300 lines of code would be needed to automate the process of producing a layer showing the best potential areas where a specific vegetation type could exist as opposed to less than 40 in a vector data format. This is because raster datasets can only hold one attribute whereas a vector dataset can hold multiple attributes. For example, a line of code first classifies areas in the precipitation surface where all pixels have an attribute greater than 50, against pixels that do not. The second line classifies areas in the precipitation surface where all pixels have an attribute less than 30 , against pixels that do not. This process continues for the minimum temperature, maximum temperature, and elevation surfaces, resulting in eight lines of code for an individual habitat type. Three habitat types exist for each vegetation type, resulting in 24 lines of code for each vegetation type. 
Thirteen different vegetation types results in a total of 312 lines of code used in the raster data model when automating this process.

In a vector data format, the automation process is much more efficient since a single dataset can hold multiple attributes. This means SQL statements can be used to select all of the attributes that fall within a precipitation, minimum temperature, maximum temperature, and elevation ranges (Figure 4-2) in one single line of code as opposed to eight in a raster format. This results in only three lines of code for each vegetation type when specifying a habitat, and a total of 39 lines of code when factoring in the 13 different vegetation types.

In addition to the feature class contained in the file geodatabase, a model was established. The model (Appendix 1) contains the "compiled_variable_data" feature class, and shows the steps performed to edit the feature class when the user enters predictive temperature degree changes and precipitation ratios. The user is also able to choose a specific vegetation (crop or forest) and habitat type through two drop down menus, which is stored in the Python script as an SQL statement (Appendix 4, Appendix 5). The SQL statement selects the specific polygons of temperature, precipitation, and elevation from the compiled_variable_data dataset associated with the chosen vegetation/habitat type and climate input specifications, then exports the selected polygons into a separate feature class storing the query results.

A script was created and implemented into the vegetation model. Like the model, the script runs all of the processes, reducing the overall processing time, and provides a much more readable and useable interface. The script's dialog box includes instructions about what temperature degree changes and precipitation ratios to enter and what the results mean in the help window on the side of the tool. The script employs the dialog box (Appendix 6), which allows the user to enter a minimum temperature degree change, enter a maximum temperature degree change, enter a precipitation ratio, select a vegetation type from a drop down menu, select a habitat type from a drop down menu, and finally save the dataset with the predicted results to a preferred destination.

In review, the Hawaiian Vegetation Model includes a file geodatabase with three main items, which include a feature class, a data model, and a script. The feature class of "compiled_variable_data" contains the original temperature, precipitation, and elevation on Hawaii. Mirror fields were created in the compiled_variable_data feature class to store the predicted variables of the estimated temperature and precipitation changes, allowing the users to compare the predicted variables with the original variables. The data model of the vegetation model displays the processing steps taken in order to create the predicted data. Finally, the script's dialog box allows the data model to become much more user-friendly by allowing the user to easily enter temperature degree changes, precipitation ratios, vegetation types, and habitat types. The reference and help dialogs in the script's dialog box instruct the users on what all of the inputs mean and what the output results indicate.

\subsection{Data Sources}

The Hawaiian Vegetation Model project gathered data from three sources. The Hawaii Coastal Geology Group had elevation data in the form of a 10-meter DEM. The Redlands Institute provided a remotely sensed vegetation dataset that included specific crop and 
forest types. Temperature and precipitation data were collected from the Western Regional Climate Centers website.

The Hawaii Coastal Geology Group consists of researchers, technicians, and graduate students from the Department of Geology and Geophysics at the University of Hawaii at Manoa. They specialize in research related to shoreline change, carbonate geology, reef geology, sedimentology, and coastal morphodynamics. Specific datasets for Hawaii offered by the Coastal Geology Group include coastal geology, shoreline imagery, USGS digital raster graphics, NOAA Landsat imagery, DEMs, LiDAR bathymetry, and various vector data. Other datasets for other Hawaiian islands are also available on the website. The Coastal Geology Group also provided elevation data to the Hawaiian Vegetation Model project in the form of a 10-meter DEM (Figure 2-5).

The Redlands Institute is made up of geospatial and technology professionals working with University of Redlands faculty. It provides datasets that explore solutions to local, regional, and global issues. The Redlands Institute uses geospatial technology to enhance research on a particular topic or study area for any subject area at any resolution. From a previous project, the Redlands Institute created a vegetation type dataset from remotely sensed data on Hawaii. The Hawaiian Vegetation Model used the vegetation dataset (Figure 4-3) (called "NRCS_CropForestRangelands" by the Redlands Institute) to compare specific crop and forest types against the temperature and precipitation datasets provided by the Western Regional Climate Center and the DEM provided by the Coastal Geology Group. 


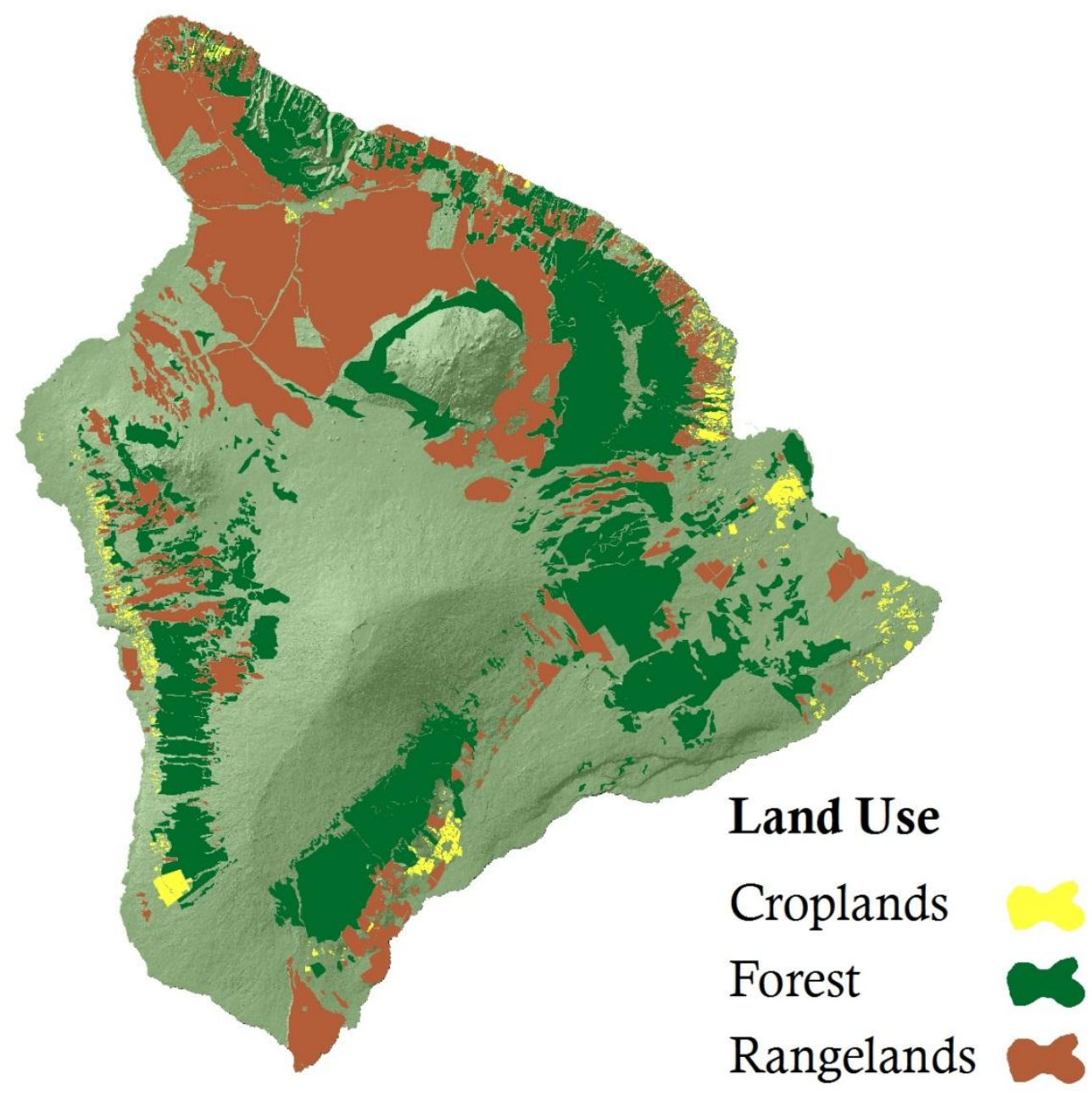

Figure 4-3: Vegetation Land Use Dataset

The Western Regional Climate Center (WRCC) provides historical atmospheric data for the states of Hawaii, Alaska, Washington, Oregon, California, Idaho, Montana, Wyoming, Nevada, Utah, Colorado, Arizona, and New Mexico. It is a branch of the Regional Climate Center (RCC), which provides climate services from national to statewide levels. The WRCC works in conjunction with the National Oceanic and Atmospheric Administration (NOAA), the National Climatic Data Center, the National Weather Service, and the American Association of State Climatologists to collect and share oceanic and atmospheric datasets. The Hawaiian Vegetation Model used temperature and precipitation datasets (Figure 2-3, Figure 2-4) from the WRCC to perform predictive future analysis on the project.

\subsection{Data Collection Methods}

The vegetation types dataset was obtained from the Redlands Institute, which obtained the dataset from the NRCS and used it for other various projects. The digital elevation 
model and the climatic data of temperature and precipitation were from alternative sources found online and downloaded at no charge. All of the collected data are from professional institutions or government agencies.

The vegetation types dataset provided the spatial information regarding crops, forests, and rangelands for Hawaii (Figure 4-3). The spatial reference system that this dataset originally used was the UTM-Zone 5N with the NAD83 datum. The NRCS compiled this dataset from Ikonos, Quickbird, and Emerge imagery, with Emerge being the primary imagery used (resolution not specified in metadata). The imagery dates range from 1999 to 2005, while the Emerge imagery dates range from 2001 to 2003 specifically. Remote sensing software was used to convert the rasterized imagery into a vector file format. To ensure accuracy of the dataset, soil conservationists verified some of the data. The Hawaiian Vegetation Model project did not use the entire NRCS_CropForestRangelands vegetation dataset. Only the individual vegetation types that selected at least 30 separate polygons from the "Compiled_Variable_Data" were used in order to avoid confusion and provide the most accurate dataset possible when comparing the vegetation types to the temperature and precipitation datasets.

The 10-meter digital elevation model (DEM) used for this project was provided by the Coastal Geology Group, which originally gathered data and created the DEM in 2007. The DEM was constructed from USGS 7.5' DEM quadrangles by first converting all of the quadrangles to a common datum and vertical unit, and then mosaicking them together. The spatial reference system of the DEM was originally NAD83 UTM-5N. The Hawaiian Vegetation Model used the DEM to gather elevation information on Hawaii. These elevation data were combined with the temperature and precipitation datasets to perform predictive analysis on the vegetation types dataset.

The remaining datasets that this project used were the temperature and precipitation datasets, which the Western Regional Climate Center provided. The datasets were displayed on a webpage as individual rain gauges with latitude and longitude points attached to each rain gauge (WGS 84). The temperature dataset was divided into an average annual maximum and annual minimum. A total of 41 rain gauges that collected weather information were used from the WRCC to observe the annual temperatures, with most points being coastal locations (Appendix 2). The precipitation dataset had 115 rain gauge stations that were used to observe annual precipitation averages (Appendix 3). The locations of the temperature and precipitation data were rain gauges, where a 30 year average of rainfall and air temperature between the years of 1971 to 2000 were used. Furthermore, on the WRCC website, each rain gauge dataset had to be individually transformed from the webpage version into a text file, cleaned up, and compiled in Microsoft Excel with the other rain gauge datasets.

\subsection{Data Scrubbing and Loading}

The data for the Hawaiian Vegetation Model required considerable scrubbing and loading in order to prepare the data. The vegetation dataset came in a shape file format that required minimal data scrubbing and loading. The attribute fields of SPC_CROP_T (specific crop type) and TYPE_FORES (forest type) were of particular interest to perform predictive analysis for the vegetation model in this dataset (Figure 4-4). However, the entire dataset did not classify every location as either a specific crop or forest but also classified some locations as an unknown crop/forest type or rangeland. 
These locations were deleted from the dataset to create a more efficient dataset with specific crop and forest types only (Figure 4-5, 4-6), to compare the elevation, temperature, and precipitation datasets to, in order to distinguish which temperature, precipitation, and elevation ranges were associated with each specific crop and forest type.

\begin{tabular}{|c|c|c|c|c|c|c|c|c|c|c|c|c|c|c|}
\hline \multicolumn{15}{|c|}{ Table } \\
\hline \multicolumn{15}{|c|}{ 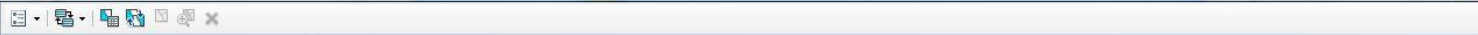 } \\
\hline \multicolumn{15}{|c|}{ NRCS_CropForestRangelands_original } \\
\hline & FID & Shape * & ID & ACRES & HECTARES & CROP_TYPE & IRRIGATION & SPC_CROP_T & LANDUSE & TYPE_FORES & Verify & Shape_area & Shape_len & A \\
\hline \multirow{4}{*}{ 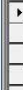 } & 0 & Polygon & 0 & 5.973263 & 2.417294 & Nuts-Tree & $Y$ & macadamia nuts & croplands & & & 24172.940413 & 774.149142 & E \\
\hline & 1 & Polygon & 0 & 2.097595 & 0.848867 & Nuts-Tree & $\mathrm{Y}$ & macadamia nuts & croplands & & & 8488.663248 & 373.997676 & \\
\hline & 2 & Polygon & 0 & 6.47787 & 2.621501 & Nuts-Tree & $\mathrm{Y}$ & macadamia nuts & croplands & & & 26215.020822 & 737.436206 & \\
\hline & 3 & Polygon & 0 & 3.491741 & 1.413057 & Nuts-Tree & Y & macadamia nuts & croplands & & & 14130.56962 & 520.831282 & \\
\hline - & 4 & Polygon & 0 & 2.3292 & 0.942594 & Bush fruit & $\mathrm{N}$ & coffee & croplands & & & 9425.927265 & 469.58459 & \\
\hline & 5 & Polygon & 0 & 5.990839 & 2.424407 & Bush fruit & Y & coffee & croplands & & & 24244.065022 & 706.894594 & \\
\hline \multirow[t]{3}{*}{ - } & 6 & Polygon & 0 & 3.806202 & 1.540315 & Bush fruit & $\mathrm{Y}$ & coffee & croplands & & & 15403.148614 & 657.663437 & \\
\hline & 7 & Polygon & 0 & 2.611197 & 1.056714 & Bush fruit & Y & coffee & croplands & & & 10567.139358 & 464.006793 & \\
\hline & 8 & Polygon & 0 & 3.016037 & 1.220547 & truck crop & Y & $\mathrm{veg} / \mathrm{mell}$ on & croplands & & & 12205.469577 & 477.557487 & \\
\hline \multirow[t]{2}{*}{ E } & 9 & Polygon & 0 & 4.660256 & 1.885939 & Bush fruit & Y & coffee & croplands & & & 18859.384302 & 737.849841 & \\
\hline & & Polygon & 0 & 2.455474 & 0.993695 & Nuts-Tree & Y & macadamia nuts & croplands & & & 9936.951257 & 650.163416 & \\
\hline \multirow{3}{*}{$E$} & & Polygon & 0 & 5.164456 & 2.089981 & Bush fruit & Y & coffee & croplands & & & 20899.806388 & 765.577705 & \\
\hline & & Polygon & 0 & 8.475802 & 3.430035 & Bush fruit & Y & coffee & croplands & & & 34300.346301 & 843.090255 & \\
\hline & & Polygon & 0 & 8.947413 & 3.620889 & Fruit-Orchards & Y & avacado & croplands & & & 36208.904143 & 857.848502 & - \\
\hline
\end{tabular}

Figure 4-4: NRCS_CropForestRangelands attribute table

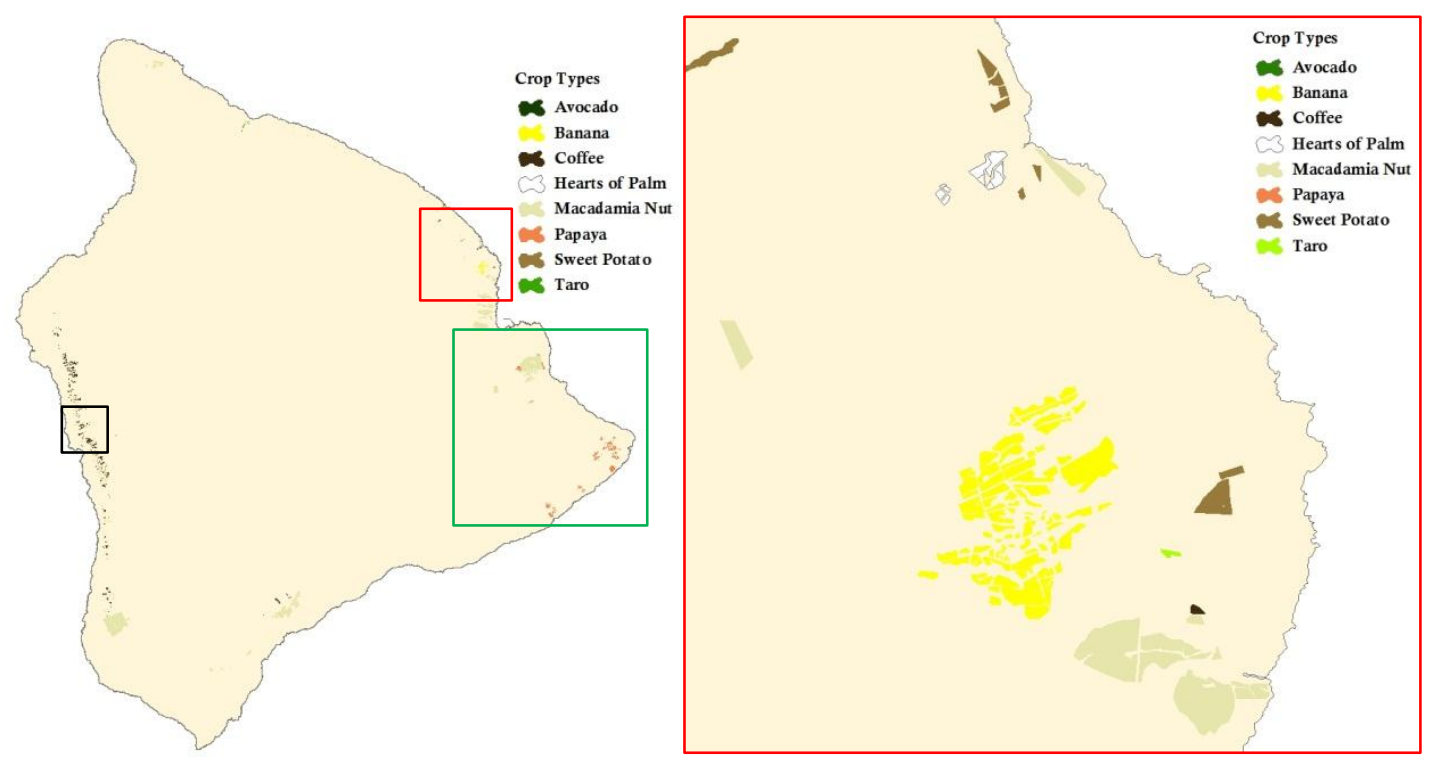




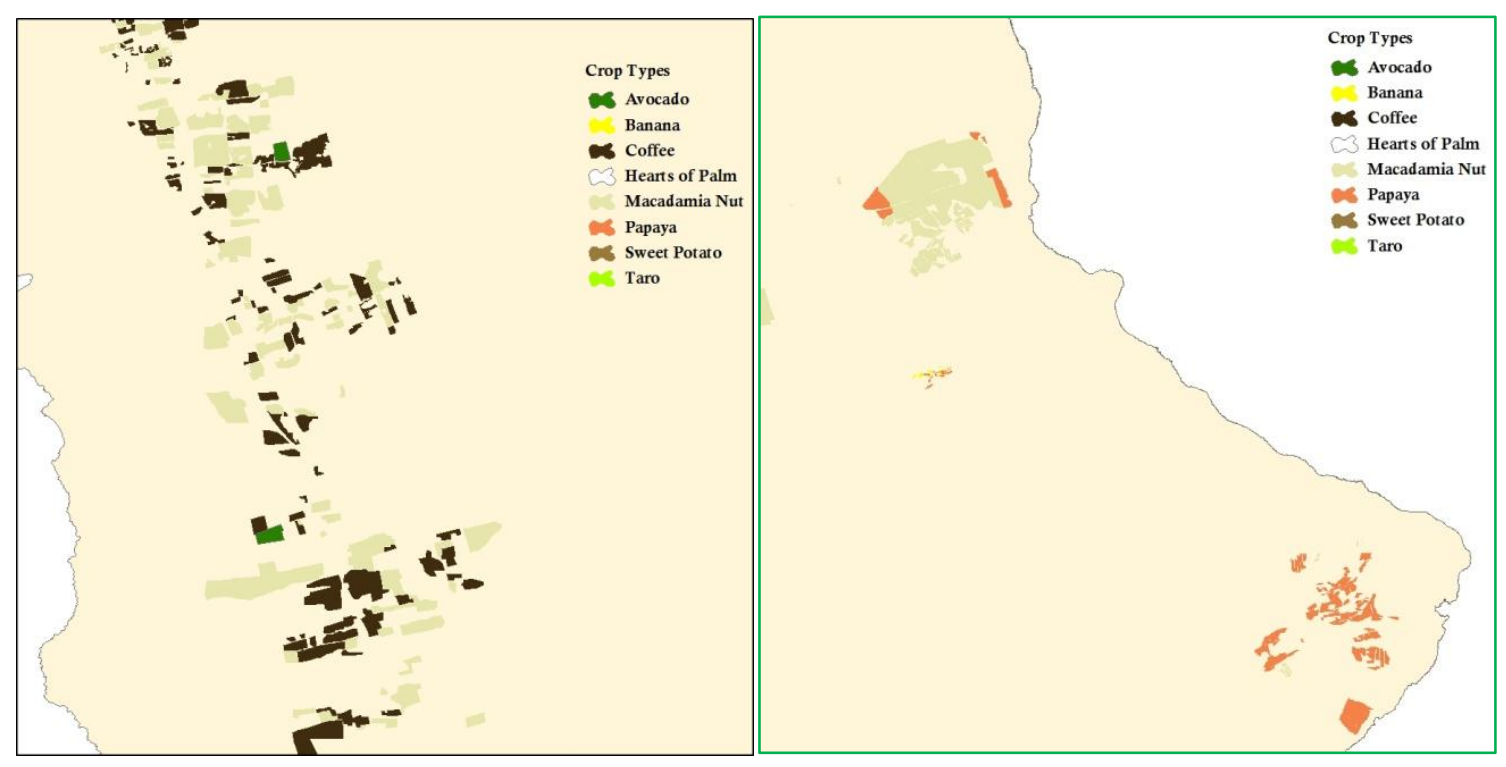

Figure 4-5: Model Crop Types 


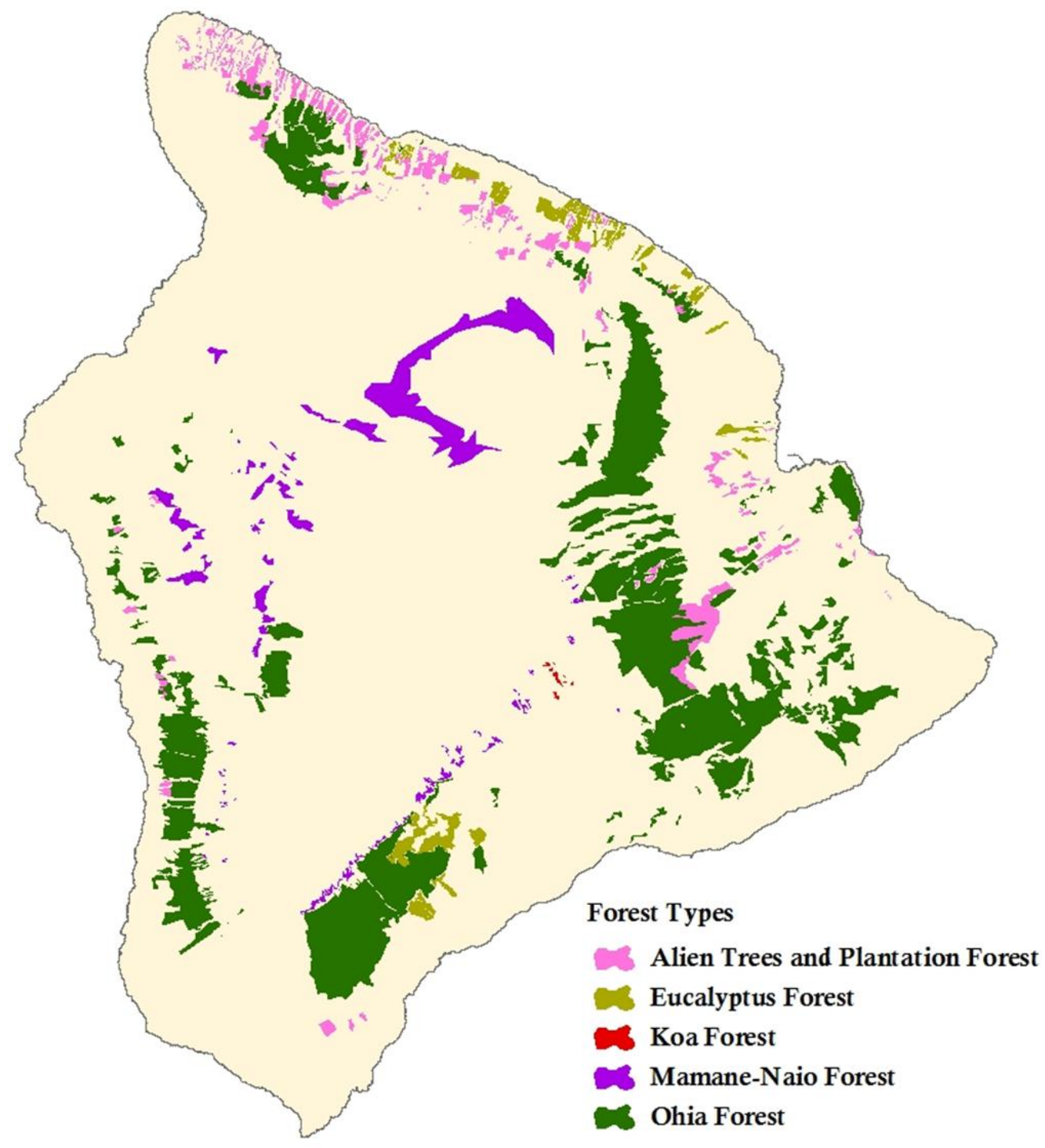

Figure 4-6: Model Forest Types

The DEM dataset gathered for this project required some data scrubbing and loading. First, considering that the model for this project was performed with vector data, the DEM had to be converted into a vector format from its original raster GRID format. Second, the elevations of the DEM were converted from meters to feet. Converting a 10meter DEM into a vector format required more work than originally expected. Without any resampling taking place, the size of the 10-meter DEM was too large to convert into a polygon vector format. Considering that the users of the Hawaiian Vegetation Model would likely view the results for the entire island at the same time, the DEM could be resampled to a larger cell size to increase the performance. The original DEM was resampled into two different cell sizes of 250 meters and 1000 meters for the entire island. The script implemented the 1000 meter dataset to decrease the processing time 
needed to execute the script. The script used the 250 meter DEM to evaluate the elevation ranges of the specific crop and forest types. This information was acquired using the 250 meter DEM for better accuracy results, but selected and drawn using the 1000 meter DEM that was aggregated into the climate datasets to decrease the processing time taken to produce the results.

The temperature and precipitation datasets were provided by the WRCC and the Hawaii Statewide GIS Program. The WRCC had 30 year averages of temperature and precipitation data for each rain gauge and the Hawaii Statewide GIS program had coordinate information for each rain gauge. The information provided by the two data sources were combined together by identifying the rain gauge station name to create a dataset that had temperature and precipitation averages associated with a geographic location (coordinate pairs).

\subsection{Summary}

The Hawaiian Vegetation Model's conceptual database design implemented elevation, temperature, precipitation rates, and vegetation types into the data model. The logical model implemented a feature class that is modified each time the model runs, a data model that graphically explains the processes of the model, and a script that provide a user-friendly interface. The data that were implemented into the model came from known educational and research institutions and were considered reliable and accurate. Some of the data collected were in shape file or rasterized formats with the exception of the temperature and precipitation data, which were in table format. Since most of the gathered data came in a format easily readable by ArcMap, minimal data scrubbing and loading took place on those datasets, with the exception of the temperature and precipitation datasets, which required substantially more work.

The purpose of the Hawaiian Vegetation Model was to figure out how temperature and precipitation rates alter the Hawaiian vegetation. Therefore, the conceptual database design centered on altering specific crop and forest types' potential locations based on temperature and precipitation rates. Elevation was also implemented into the model, but this variable is static, considering that significant differences in elevations rarely change within a year.

The logical data model of this project included a feature class, a data model, and a script located in the file geodatabase. The feature class included the attributes of average temperatures, average precipitation rates, and elevations, along with mirror fields to store the predicted attributes created from the model's processes. The data model displayed the steps of how the model's processes ran. The file geodatabase also included a script dialog box that ran the script separately. The scripting dialog also provided instructions on what to input and what the results would be, while decreasing the processing times.

The vegetation dataset used in this project was provided by the Redlands Institute. The DEM used in this project was provided by the Coastal Geology Group, which is in conjunction with the University of Hawaii at Manoa. Finally, the Western Regional Climate Center (WRCC) provided the temperature and precipitation datasets. The WRCC datasets were compiled from a variety of sources including the National Oceanic and Atmospheric Administration and the National Weather Service.

Data collection methods for the Hawaiian Vegetation Model included searching websites and gathering the data manually, with the exception of the vegetation dataset. 
The vegetation dataset was provided by the Redlands Institute. The digital elevation model was downloaded from the Coastal Geology Groups website in a GRID raster format. Finally, the temperature and precipitation datasets were individually transformed into text files from the WRCC website. Each rain gauge station had to be viewed separately, but temperature and precipitation information were available for a selected amount of stations throughout Hawaii.

Data scrubbing and loading was required on each of the datasets gathered for the Hawaiian Vegetation Model. The specific crop and forest types were extracted from the vegetation dataset to reduce the amount of undesirable information located within that dataset. The DEM was converted from a raster format to a vector format so it could be aggregated to the joined temperature and precipitation datasets. The temperature and precipitation datasets were originally transformed into text files from the WRCC website, converted, compiled, cleaned up in Microsoft Excel, and joined with another rain gauge table which provided coordinates that are more accurate. 



\section{Chapter 5 - Implementation}

\subsection{Introduction}

The Hawaiian Vegetation Model was created through a four-step process. In the first step, each dataset was individually modified to prepare it for the vector based data model. The second step used the modified datasets to discover the temperature, precipitation, and elevation ranges that each crop and forest type were associated with. For example, when discovering precipitation ranges for the banana crop type, the middle 40 percent of banana plantations were in areas with average annual precipitation rates between 207 to 209 inches. The third step included constructing the vegetation model and script dialog in ArcGIS's Model Builder application and Python. The fourth step visually compared all of the interpolated raster surfaces against the two most common interpolation methods of kriging and IDW.

The first step of dataset modification comprised preparing all the datasets to be inserted into what was to be the vector based vegetation model. The model would incorporate the datasets of temperature, precipitation, elevation, and vegetation types. The temperature and precipitation datasets were converted to raster surfaces by interpolation, while the DEM was provided as a raster surface. The raster surfaces were converted to a vector format so that the three datasets could be joined. The vegetation dataset was overlaid onto the compiled aggregate dataset using the select by location and select by attribute tools in conjunction.

Once the dataset preparatory work was completed, the climate and elevation ranges were identified, so they could be implemented into the vegetation model as SQL expressions. Specific crop and forest type ranges were identified by taking the vegetation land use feature class (Figure 4-3) and overlaying this layer onto the aggregated polygon datasets of minimum temperature, maximum temperature, precipitation, and elevation. Once the vegetation land use feature class was overlaid onto the aggregated polygon dataset, each specific crop or forest type was individually selected in the vegetation land use dataset using the select by attribute tool (for example, all coffee plantations were selected). Any polygon from the aggregated dataset that intersected each individual selected crop or forest type were then selected using the select by location tool (for example, all polygons from the aggregated dataset that intersected the coffee plantations were selected). The selected attributes of the aggregate dataset of each vegetation type were exported and opened in Microsoft Excel and SPSS to discover the specific climate and elevation ranges for each vegetation type using a percentile method.

Once the climate and elevation ranges were found for each vegetation type, the third step of creating the model could take place. The temperature, precipitation, and elevation datasets (1000 x 1000-meter resampled DEM) were compiled into one large aggregate dataset as the base layer of the predictive analysis. The dataset was added to the Model Builder window, along with three calculate field tools, a select tool, a dissolve tool, three VBA expression boxes connected to the calculate field tools, and a SQL expression box connected to the select tool (Appendix 1). The elements were arranged in a manner by which a user predicts the minimum temperature change, the maximum temperature change, and the precipitation change in the VBA expression boxes. The user 
then selects the desired vegetation and habitat type in the SQL expression box before running the modeling processes. A script dialog box was included for the model (Appendix 6) to provide instruction, as well as to provide the users with a simpler interface than the interface provided by Model Builder.

The fourth step included testing the accuracy of the interpolated raster datasets of minimum temperature, maximum temperature, and precipitation. This was accomplished by creating and visually comparing the two most common interpolation methods of ordinary kriging and inverse distance weighted (IDW) for each of the three datasets. The ordinary kriging method was chosen for all three of the datasets over the IDW method because various outside research had found ordinary kriging to be more accurate than the IDW method (Kravchenko \& Bullock, 1999). In addition, an ordinary kriging error surface map was generated for each of the three datasets to give a perspective of where the least and most accurately interpolated values exist. The inland areas on the western side generated areas with the highest error for both temperature and precipitation. The coastal areas and the inland areas on the eastern side of the island had the lowest errors.

\subsection{Dataset Modification}

Before all of the datasets could be implemented into the vector data model, certain preparatory work had to be performed on the datasets. This project attempted to perform the modeling analysis in a vector data format as opposed to a traditional raster data format. The temperature and precipitation datasets had to be rasterized from point data and then transformed into a polygon vector format before they could be aggregated to both of the resampled DEMs in a vector format. It was necessary to convert the raster surfaces into vector surfaces so multiple attributes could be added to a singular dataset and then perform a predicted analysis on it. The elevation dataset that was originally provided in a raster format was first resampled into a $250 \times 250$ meter DEM and resampled again into 1000 x 1000 meter DEM, which were then converted into a vector polygon format so that this dataset could be aggregated to the polygon datasets of temperature and precipitation. When the DEM was converted into a vector format, the polygons represented each pixel value when it was in a raster format. This means each elevation polygon was represented by a $250 \times 250$ or $1000 \times 1000$ meter square in the vector version of the DEM dataset.

\subsubsection{Climate Dataset Modification}

The temperature and precipitation datasets had to first be rasterized from the point data as the first step to merging all of the datasets together. The same points (rain gauges) represented both temperature averages and precipitation rates. One-hundred fifteen rain gauges collected precipitation averages across the island, but only 41 of these points contained temperature averages. To create an interpolated raster surface from these points, kriging was used. Although an interpolated surface is just an estimate surface, the kriging algorithm allowed the precipitation and temperature averages to spread over the entire surface of Hawaii as a continuous surface. Once the kriging algorithm was applied, it was noticed that not every area on Hawaii was represented with temperature and precipitation information. Small areas on the eastern and southern tips of the island were without temperature information (no data) because the extent of the data points did not 
reach those areas (Figure 2-3). A small area on the eastern side of the island as well as a substantially larger area on the southern tip of the island had no minimum and maximum temperature information (Figure 2-4).

Once the raster surfaces were created, they had to be converted back into a vector format (Figure 5-1, Figure 5-2). The vector surfaces allowed multiple attributes to be included in the attribute table, as well as allow the surfaces to be overlaid onto the vegetation dataset to study the temperature and precipitation ranges associated with each specific crop or forest type. In order to convert the created kriged surfaces into a polygon format, the cell values had to first be converted to integers. This was achieved by adding 0.5 to all values and then using the truncate tool.

Once the precipitation and temperature datasets were in integer format, the raster to polygon tool was run to create three vector datasets. The three vector datasets were then joined into a single vector dataset using the identity tool, located in the Analysis toolbox. Once the large aggregate dataset was created, the temperature and precipitation data were ready to be overlaid against the vegetation types dataset to discover each crop and forest type's temperature and precipitation ranges. 


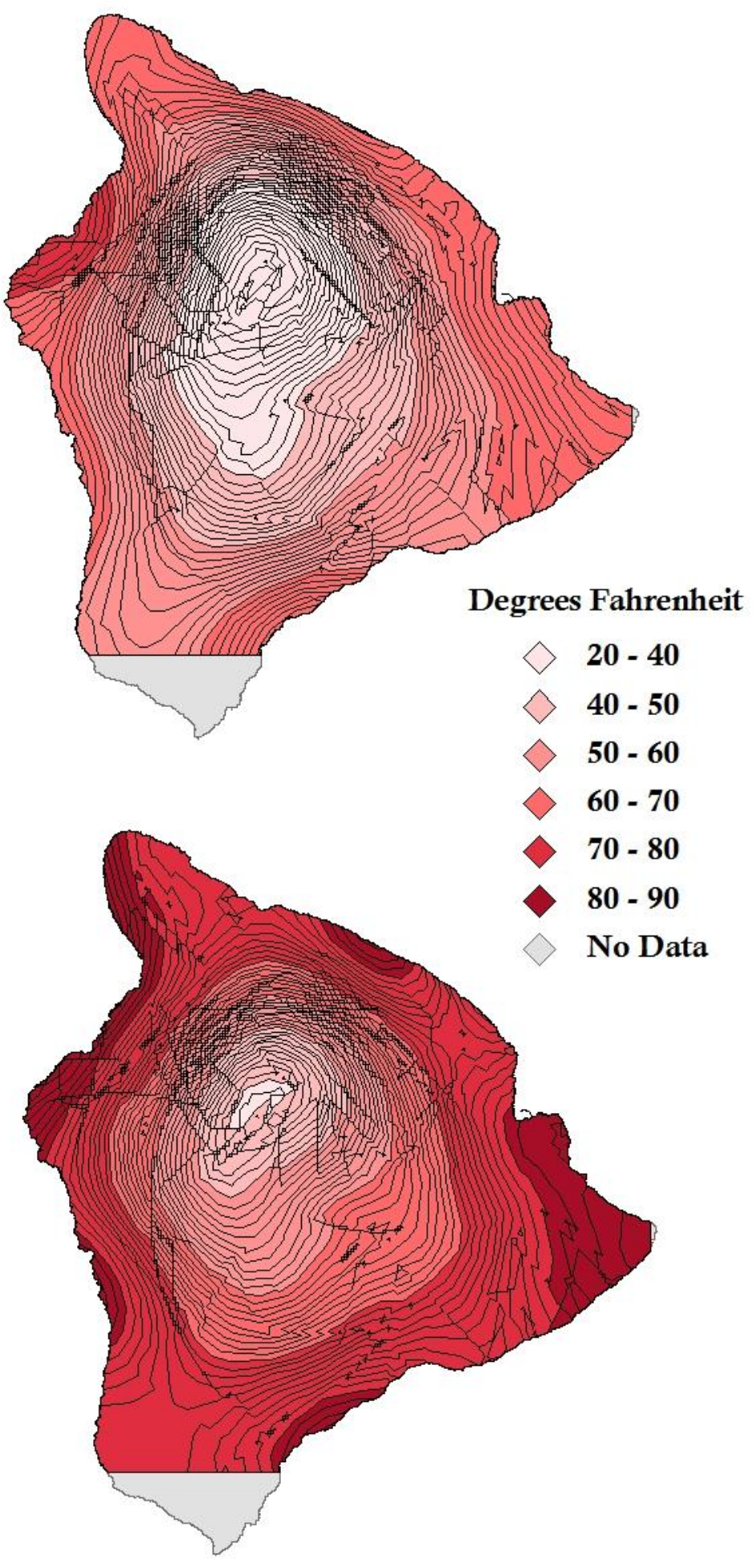

Figure 5-1: Vector formats of minimum (top) and maximum (bottom) temperature 


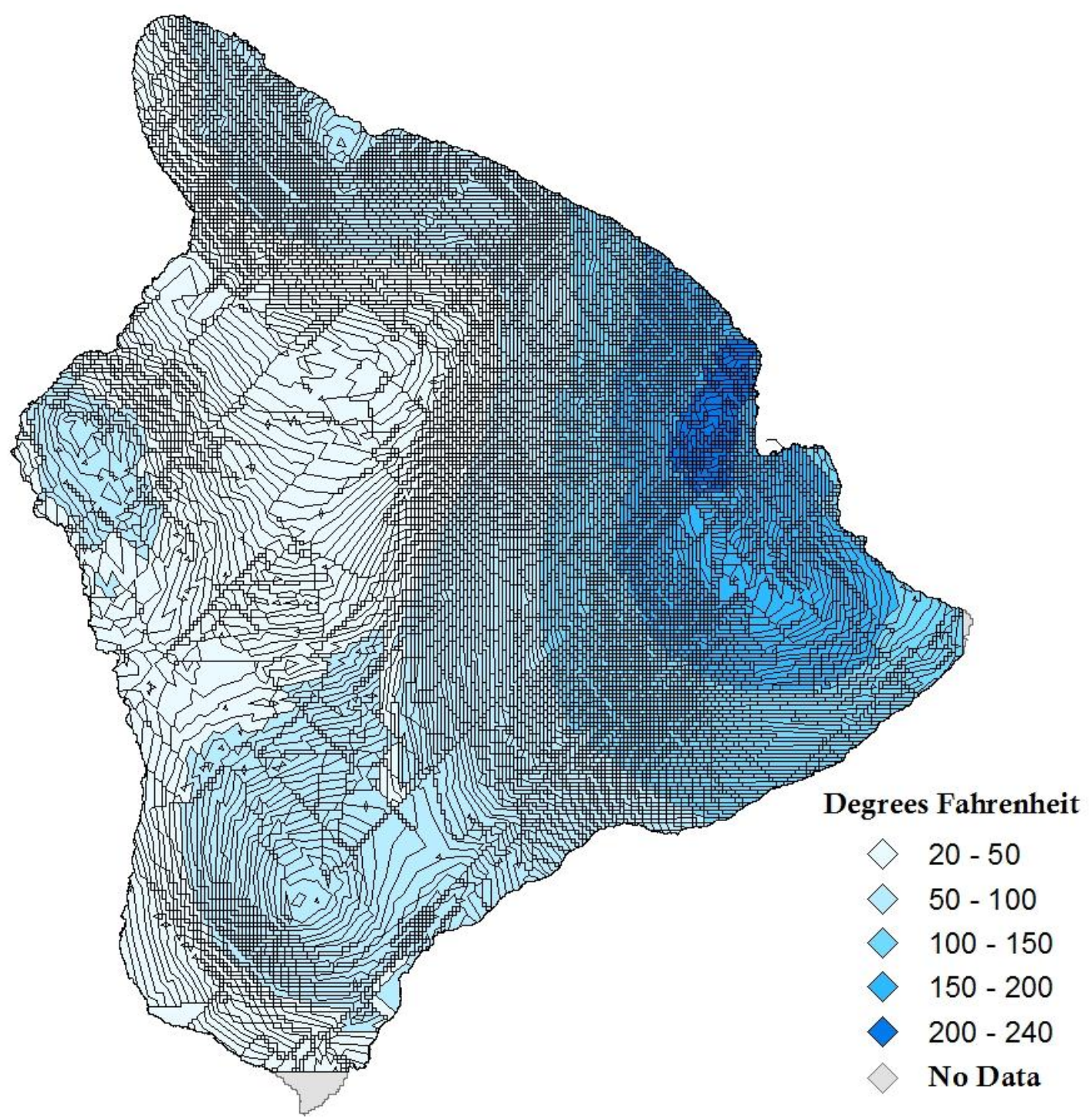

Figure 5-2: Vector format of precipitation

\subsubsection{Elevation Dataset Modification}

The original elevation dataset was downloaded from the Coastal Geology Group's website as a 10-meter DEM. This dataset underwent a series of resampling test trials to determine which resolution would be best for the project. On the first test trial, the 10meter DEM was not resampled and the 10-meter pixel resolution was left to be converted into a vector format without any changes. The 10-meter DEM first had to be converted into an integer format, since the elevation values were in a floating number format. The Raster Calculator tool and the Int tools rounded and converted the floating numbers into integers. The raster to vector conversion could now take place using the raster to polygon tool in the Conversion Tools toolbox in ArcMap. The dataset converted from a raster 
format to a vector format without a problem. However, when using the identity tool to join the elevation dataset to the aggregated climate dataset from the previous section (section 5.2.1), the dataset was too large, as the processing was terminated after several hours.

The second attempt resampled the 10-meter DEM into a 20-meter DEM to see if halving the resolution would allow the raster to polygon tool to complete its processing. The first step was to use the resampling tool in the Raster toolbox to resample the 10meter DEM into 20 meters, using the "nearest neighbor" method. This method was selected over the bilinear, cubic, and majority methods to avoid any kind of smoothing and uphold the accuracy of the dataset as much as possible (Soille et al., 2003). The dataset was then ready to go through the Raster Calculator and Int tools to convert the floating numbers to integers, and the raster to polygon tool to convert the raster surface into a vector surface. This was again a success, but when using the identity tool to join the elevation dataset to the aggregated climate dataset, the dataset was again too large, as the processing took more than two hours to complete (identity tool terminated after two hours).

Not wanting to experiment any further, the rasterized version of the 100-foot contour surface's (the elevation dataset used before discovering the 10-meter DEM) metadata was opened to find out the pixel size. After filing through the metadata, the pixel values were discovered to be around 513 meters (Figure 5-3). Important to note was that the vegetation model's overall processing time was around 34 seconds with this pixel value. This sparked the idea to resample the DEM from 10-meters to 250-meters to extract the elevation ranges that were incorporated into the SQL statements, but resample the 10-meter DEM again to 1000-meters to reduce the drawing times it took the vegetation model to perform the analysis. The resampled 250-meter and 1000-meter DEMs went through the same processes as the 10- and 20-meter DEMs in the previous paragraph (resampling tool using nearest neighbor, int tool, and raster to polygon tool) to convert the raster surfaces into vector polygon surfaces (Figure 5-4). The resampled 250meter DEM was then joined to the aggregated vector polygon climate dataset using the identity tool in the previous section (5.2.1) and then overlaid onto the vegetation land use dataset using the select by location and select by attributes tools to gather the elevation ranges of each specific crop and forest type and store them as SQL statements. The 1000meter DEM was joined to a separate version of the aggregated climate dataset (discussed in section 5.2.1) to reduce the overall processing/drawing times the model took to create results, compared against the resampled 250-meter DEM. Specifically, using the 1000meter DEM instead of the 250-meter DEM reduced the total processing times from about 78 seconds with the 250-meter DEM to about 29 seconds with the 1000-meter DEM (when running the model with coffee as the selected vegetation type) (Figure 5-5). 


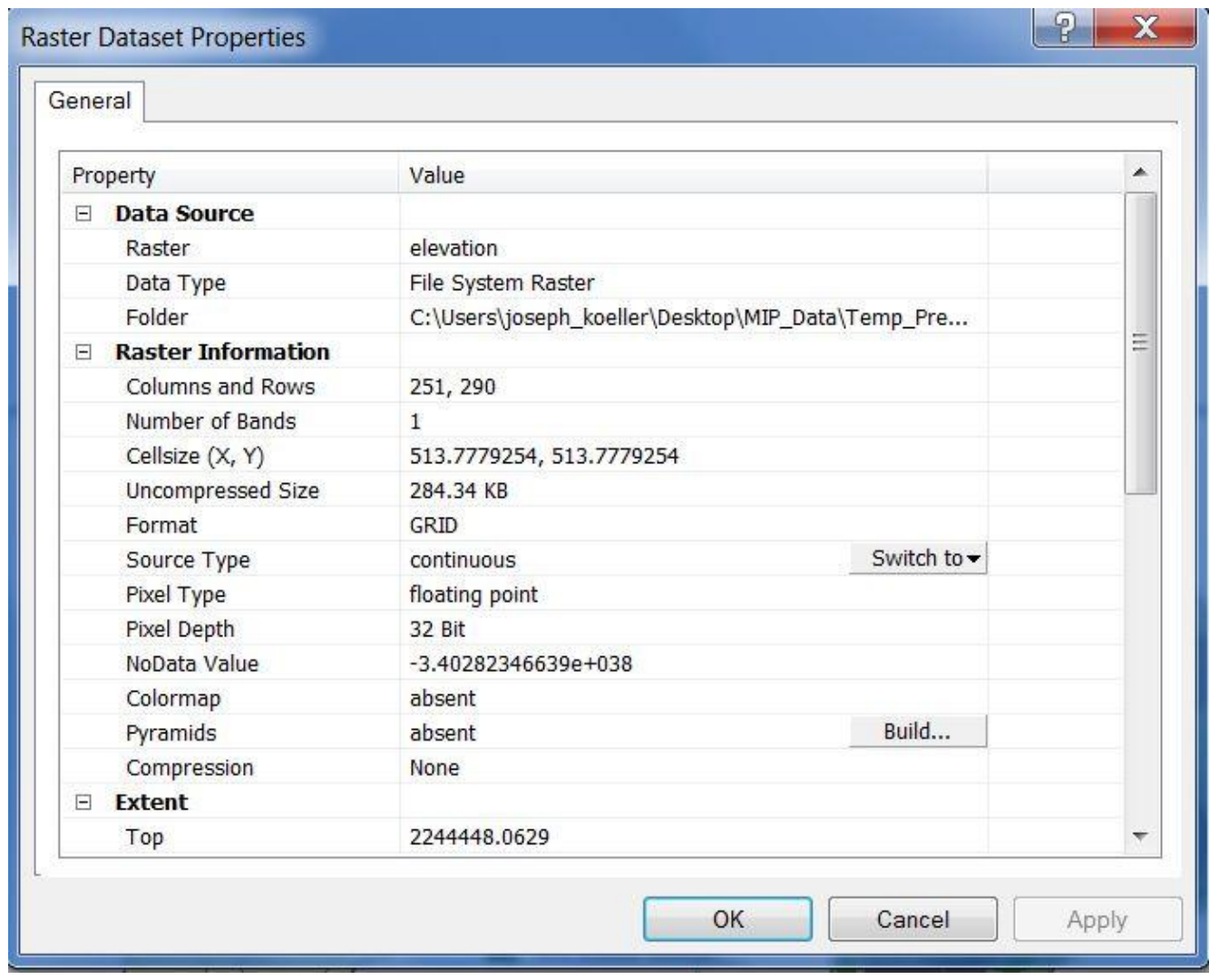

Figure 5-3: Metadata of the 100-foot contours elevation dataset 


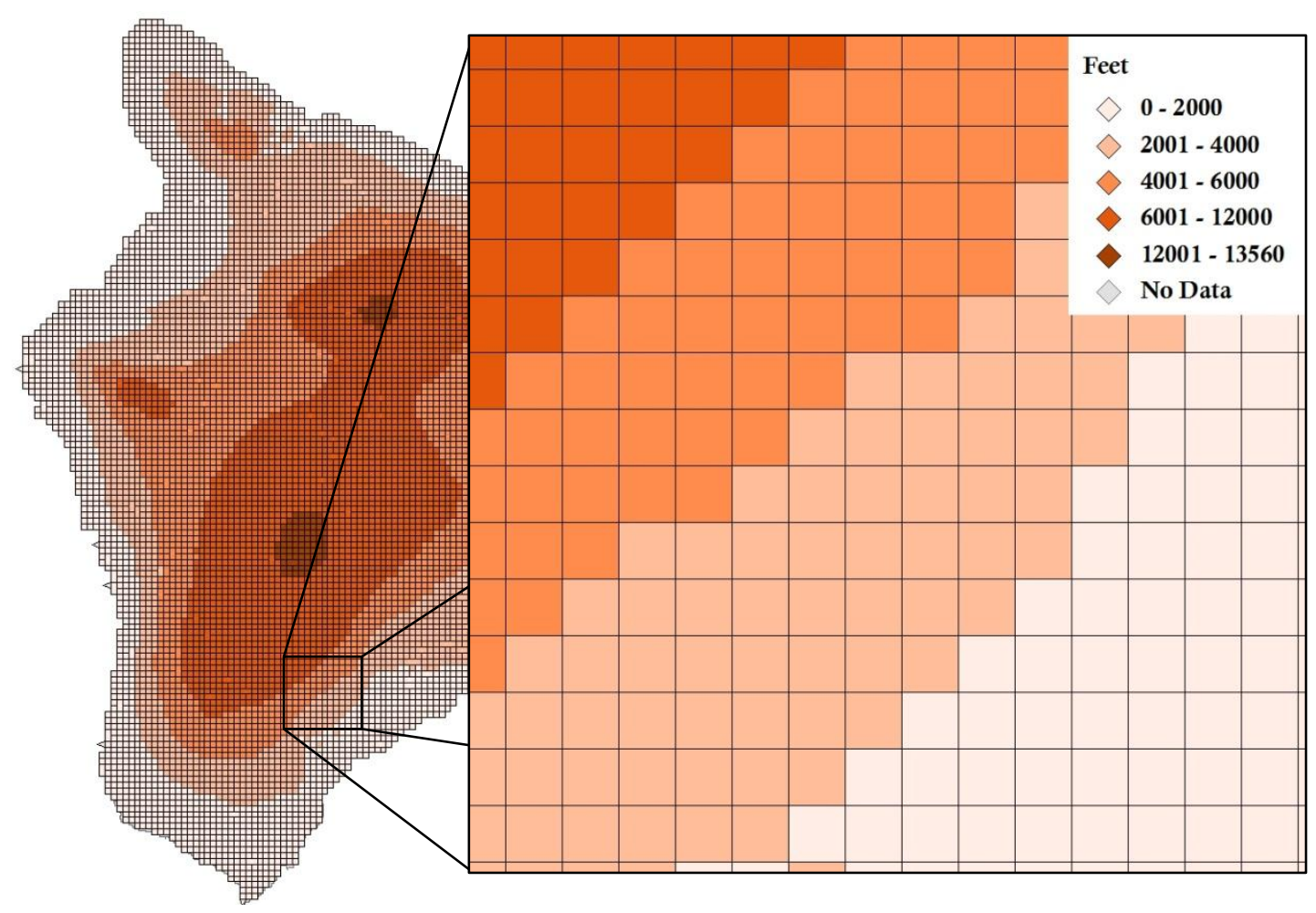

Figure 5-4: Vector format of the resampled 1000-meter DEM

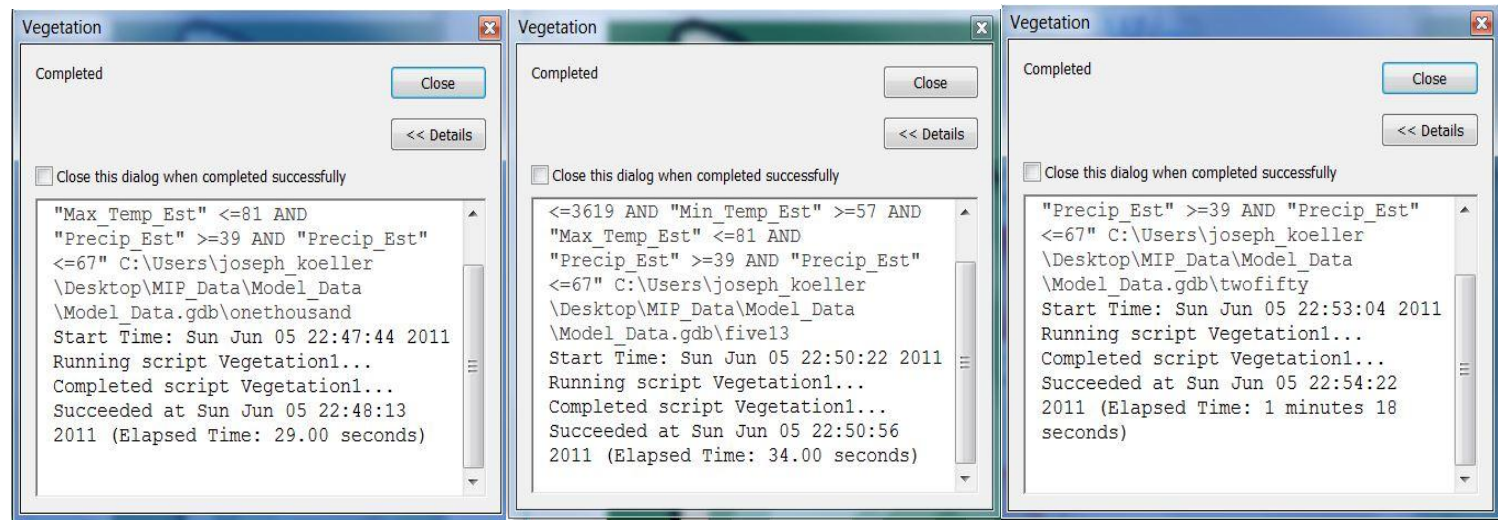

Figure 5-5: Comparison between the resampled datasets 


\subsubsection{Dataset Modification Summary}

In review, preparatory work was done on each of the datasets in order to compare the vegetation types dataset against the aggregated datasets of minimum temperature, maximum temperature, precipitation, and elevation. The temperature datasets, represented in a vector point format, had to first be rasterized to apply temperature values across the majority of Hawaii's surface. They were then converted into a vector polygon format so they could be joined with the precipitation and elevation datasets and compared against the vegetation types dataset. The precipitation dataset followed the same process as the temperature datasets, in which the vector points had to be interpolated into a raster surface and then converted back into a vector surface represented in a polygon format. The elevation dataset was resampled into a 250- and 1000-meter DEM from its original 10-meter DEM. The resampled DEMs were then converted into a polygon format, where the 250-meter DEM was used to discover the each vegetation types climate and elevation range and the 1000-meter DEM was implemented into the vegetation model to reduce the processing and drawing times when the model was run. Figure 5-6 shows the four individual attributes contained in a single polygon once the datasets of minimum temperature, maximum temperature, precipitation, and elevation were aggregated together to create on large dataset.

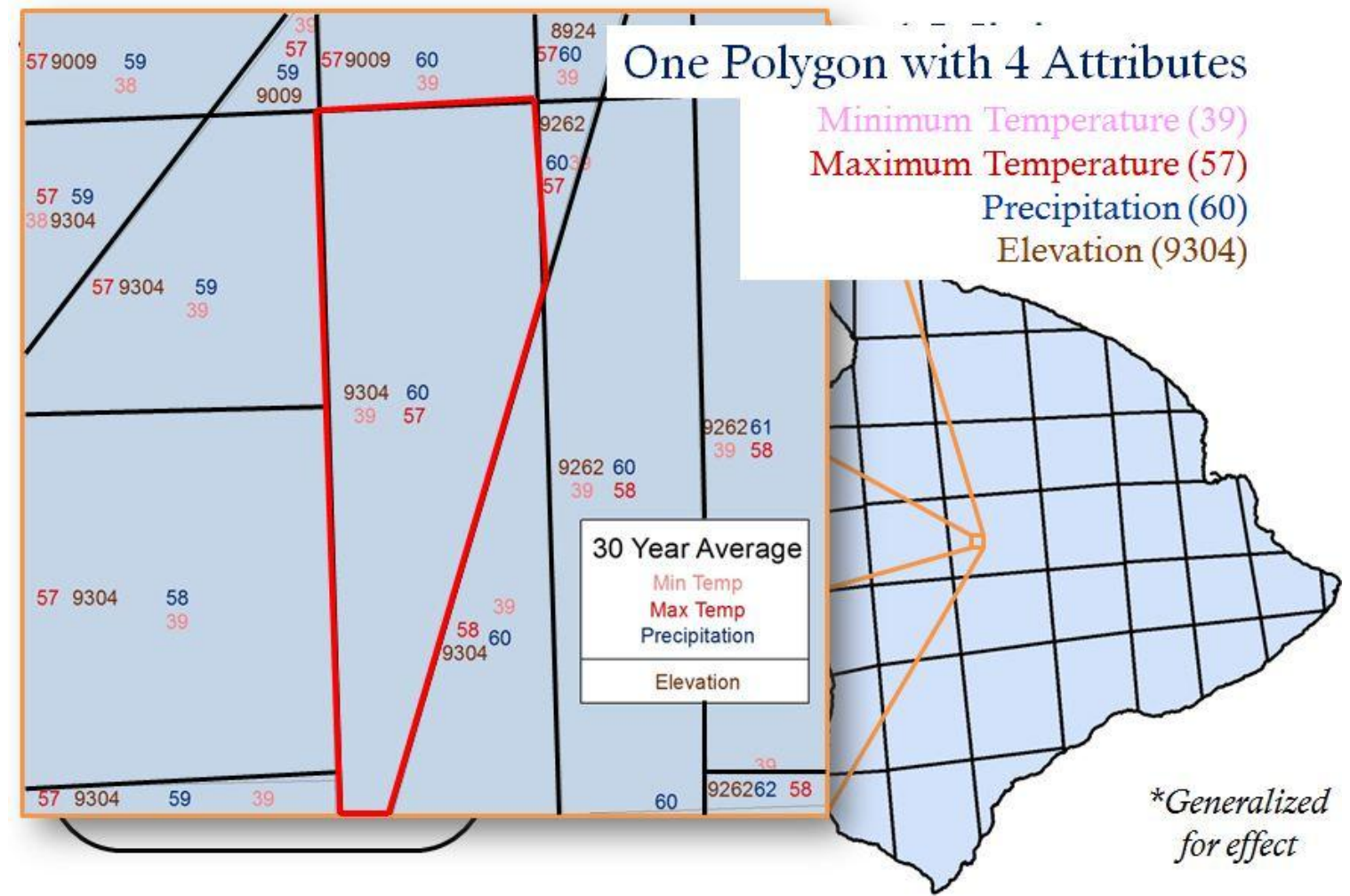

Figure 5-6: Attributes of the aggregated climate and elevation dataset 


\subsection{Vegetation Ranges Discovery}

Once the datasets of temperature, precipitation, and elevation were aggregated together in a polygon vector format, they could be compared against the vegetation types dataset to discover the temperature, precipitation, and elevation ranges associated with each specific crop and forest type. The temperature dataset actually included two datasets. Since the temperature averages downloaded from the WRCC website were only provided in average minimum and average maximum, both variables had to be interpolated, converted into their own vector surfaces, and then aggregated to the precipitation and elevation polygon datasets before the temperature attributes could be compared against the vegetation types dataset. The precipitation data was available as annual precipitation, which was used as the precipitation attribute in the aggregate dataset, when the aggregate dataset was compared against the vegetation types dataset. Once elevation was joined to the aggregated dataset, the elevation attribute was also compared against the vegetation types dataset because elevation differences on Hawaii can change drastically in a short horizontal distance.

\subsubsection{Vegetation Dataset Comparison}

Once the minimum temperature, maximum temperature, precipitation, and elevation datasets were joined together into one large aggregated dataset (section 5.2), they were compared against the vegetation types dataset (Figure 4-3) to discover the specific climate and elevation ranges associated with each crop and forest type. However, it was originally noticed that some of the polygons were very elongated and very large. The initial thought was to manually start an edit session to chop up all of the long and large polygons, convert the polygons to a point format, and then perform a spatial join of these polygons to each of the temperature, precipitation, and elevation polygon datasets. The next idea was to use the identity tool, which subdivided and joined the attributes of all the irregular shaped polygons while additionally preserving the accuracy without using an edit session to subdivide the polygons. However, both the select by attribute and select by location tools were used in conjunction to select all of the polygons from the aggregated dataset that intersected with a specific vegetation type (Figure 5-7) to discover the minimum and maximum temperature, precipitation, and elevation percentile ranges for each specific vegetation type. 


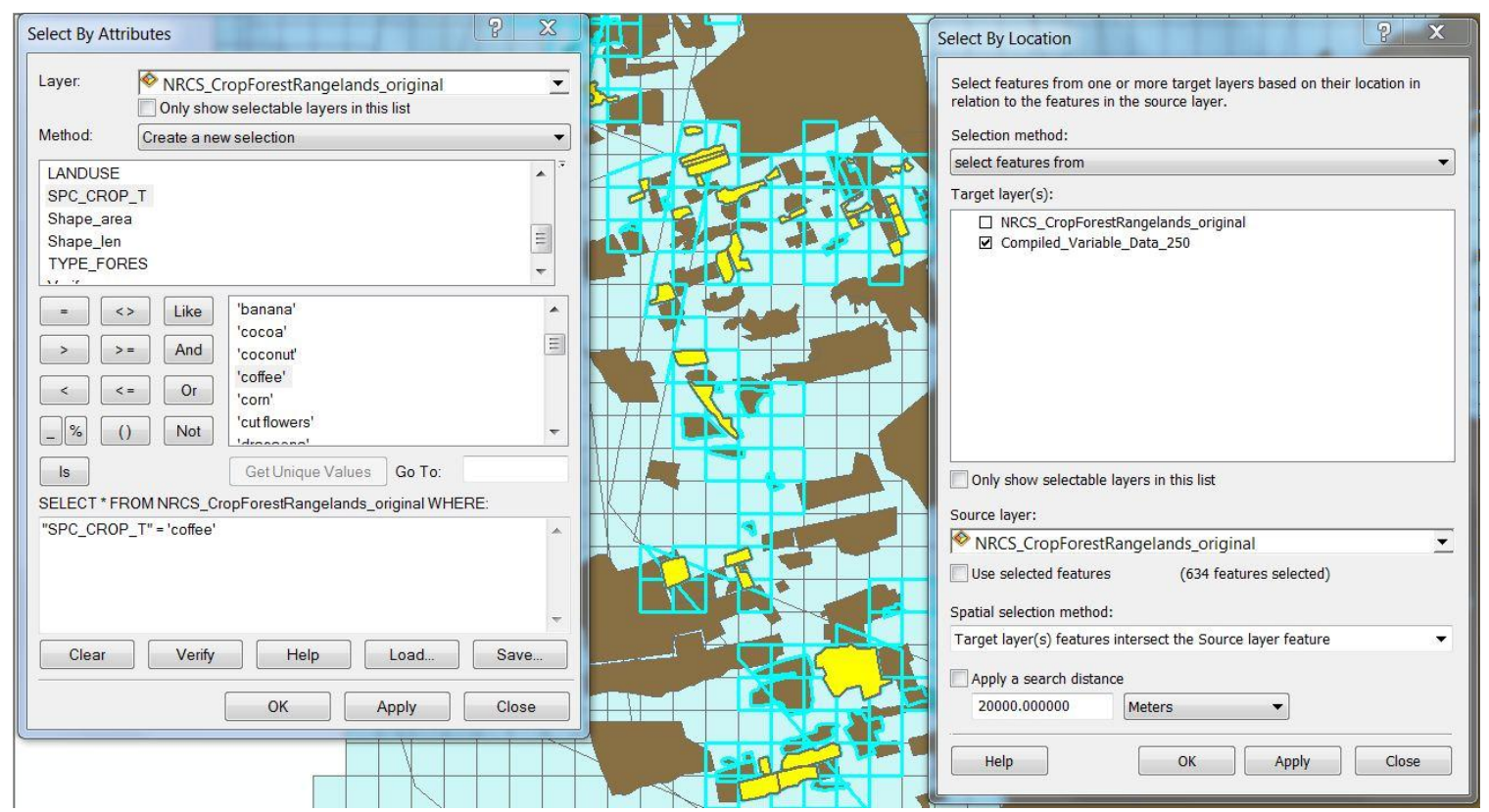

Figure 5-7: Select by attributes and select by location tool working in conjunction

The select by attribute tool selected an individual crop or forest type, while the select by location tool selected each polygon of the aggregated climate and elevation dataset (sections 5.2.1 and 5.2.2) that intersected with the polygons of the specific selected crop or forest type. Once all of the polygons of a specific vegetation type were selected from the aggregated dataset (Figure 5-7), the selected attributes of the aggregated dataset were exported into Microsoft Excel and SPSS. SPSS was used to discover the percentile ranges associated with each specific crop and forest type. This discovered a specific minimum temperature, maximum temperature, precipitation, and elevation value range for each specific vegetation type. For example, when the attributes of the selected polygons of the aggregated dataset that had intersected with coffee plantations were brought into SPSS, the $30^{\text {th }}$ and $70^{\text {th }}$ percentile (middle 40 percent of the data with the values arranged in descending or ascending order) were calculated to be 47 and 51 inches respectively, for the precipitation attribute (Figure 5-8). The percentile point ranges of 40,65, and 90 percent were identified for each of the four attributes of annual minimum temperature, annual maximum temperature, annual precipitation, and elevation for each specific crop and forest type (Table 5-1). It is important to note that the table shows the temperature ranges in degrees Fahrenheit, the precipitation ranges in inches, and the elevation ranges in feet. 


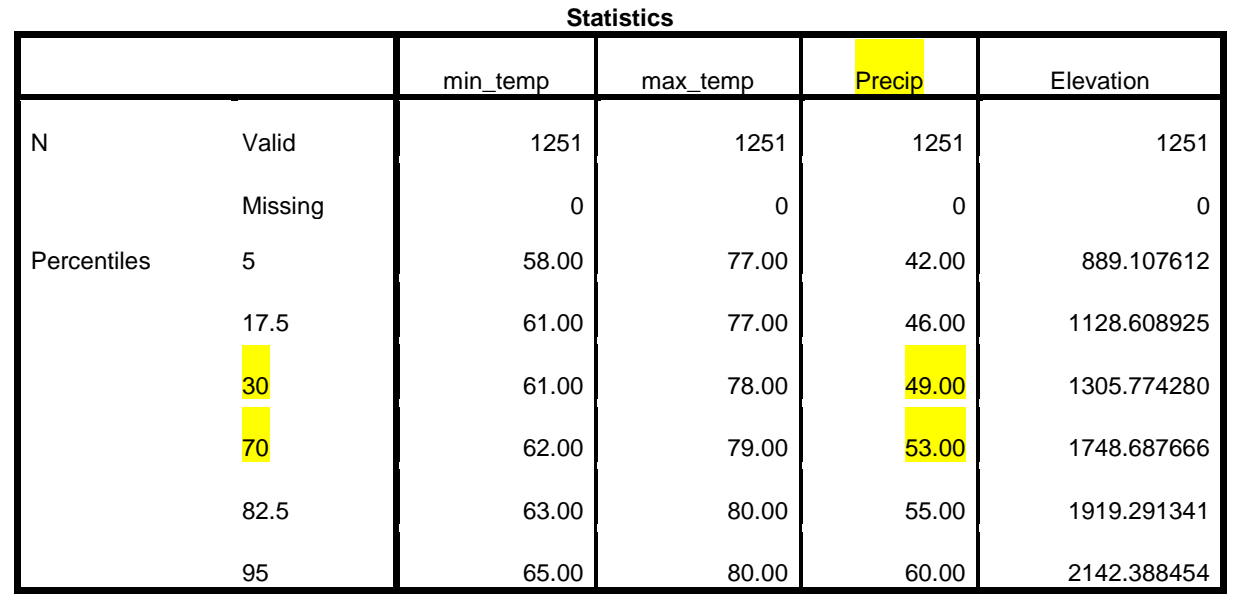

Figure 5-8: The SPSS output of the percentile points percent values of coffee

Table 5-1: Summary statistics for each dataset and each vegetation type

\begin{tabular}{|c|c|c|c|c|c|c|c|c|}
\hline $\begin{array}{c}\text { Middle } \\
\text { Percent of } \\
\text { Data } \\
\begin{array}{c}\text { Around } \\
\text { Median }\end{array}\end{array}$ & $\begin{array}{c}\text { Min } \\
\text { Temp } \\
\text { Lower } \\
\text { Percent- } \\
\text { ile }\end{array}$ & $\begin{array}{c}\text { Min } \\
\text { Temp } \\
\text { Upper } \\
\text { Percent- } \\
\text { ile }\end{array}$ & $\begin{array}{c}\text { Max } \\
\text { Temp } \\
\text { Lower } \\
\text { Percenti } \\
\text { le }\end{array}$ & $\begin{array}{c}\text { Max } \\
\text { Temp } \\
\text { Upper } \\
\text { Percent- } \\
\text { ile }\end{array}$ & $\begin{array}{c}\text { Precip } \\
\text { Lower } \\
\text { Perce- } \\
\text { ntile }\end{array}$ & $\begin{array}{c}\text { Precip } \\
\text { Upper } \\
\text { Perce- } \\
\text { ntile }\end{array}$ & $\begin{array}{c}\text { Elev } \\
\text { Lower } \\
\text { Perce- } \\
\text { ntile }\end{array}$ & $\begin{array}{c}\text { Elev } \\
\text { Upper } \\
\text { Perc- } \\
\text { entile }\end{array}$ \\
\hline $\begin{array}{c}\text { Avocado } \\
40 \%\end{array}$ & 59 & 62 & 77 & 80 & 44 & 48 & 1132 & 1627 \\
\hline $\begin{array}{c}\text { Avocado } \\
65 \%\end{array}$ & 59 & 62 & 77 & 80 & 44 & 48 & 1096 & 1701 \\
\hline $\begin{array}{c}\text { Avocado } \\
90 \%\end{array}$ & 59 & 63 & 77 & 81 & 43 & 49 & 1004 & 1759 \\
\hline Banana 40\% & 64 & 64 & 76 & 77 & 207 & 209 & 617 & 787 \\
\hline Banana 65\% & 64 & 64 & 76 & 77 & 205 & 210 & 554 & 840 \\
\hline Banana 90\% & 64 & 65 & 75 & 81 & 167 & 211 & 353 & 981 \\
\hline Coffee 40\% & 62 & 62 & 77 & 79 & 47 & 51 & 1266 & 1732 \\
\hline Coffee 65\% & 61 & 63 & 76 & 80 & 46 & 54 & 1125 & 1919 \\
\hline Coffee 90\% & 59 & 66 & 75 & 81 & 44 & 60 & 870 & 2152 \\
\hline $\begin{array}{c}\text { Hearts of } \\
\text { Palm 40\% }\end{array}$ & 63 & 63 & 74 & 75 & 156 & 195 & 246 & 1414 \\
\hline $\begin{array}{c}\text { Hearts of } \\
\text { Palm 65\% }\end{array}$ & 63 & 64 & 74 & 75 & 155 & 197 & 233 & 1448 \\
\hline $\begin{array}{c}\text { Hearts of } \\
\text { Palm 90\% }\end{array}$ & 63 & 64 & 74 & 75 & 156 & 197 & 51 & 1597 \\
\hline & & & & & & & & \\
\hline
\end{tabular}




\begin{tabular}{|c|c|c|c|c|c|c|c|c|}
\hline $\begin{array}{l}\text { Macadamia } \\
\text { Nuts } 40 \%\end{array}$ & 62 & 65 & 78 & 80 & 47 & 156 & 646 & 1385 \\
\hline $\begin{array}{l}\text { Macadamia } \\
\text { Nuts } 65 \%\end{array}$ & 60 & 66 & 76 & 81 & 46 & 194 & 344 & 1722 \\
\hline $\begin{array}{l}\text { Macadamia } \\
\text { Nuts } 90 \%\end{array}$ & 58 & 67 & 76 & 82 & 43 & 204 & 108 & 2195 \\
\hline Papaya $40 \%$ & 67 & 68 & 83 & 84 & 141 & 146 & 141 & 305 \\
\hline Papaya 65\% & 66 & 68 & 83 & 84 & 137 & 148 & 79 & 445 \\
\hline Papaya $90 \%$ & 65 & 68 & 82 & 84 & 132 & 168 & 49 & 661 \\
\hline $\begin{array}{c}\text { Sweet } \\
\text { Potato } 40 \%\end{array}$ & 63 & 64 & 74 & 74 & 176 & 194 & 175 & 380 \\
\hline $\begin{array}{c}\text { Sweet } \\
\text { Potato } 65 \% \\
\end{array}$ & 63 & 65 & 73 & 77 & 173 & 197 & 145 & 679 \\
\hline $\begin{array}{c}\text { Sweet } \\
\text { Potato } 90 \% \\
\end{array}$ & 63 & 66 & 72 & 79 & 160 & 201 & 94 & 946 \\
\hline Taro $40 \%$ & 63 & 65 & 77 & 77 & 97 & 98 & 30 & 135 \\
\hline Taro $65 \%$ & 63 & 65 & 76 & 77 & 97 & 160 & 26 & 292 \\
\hline Taro $90 \%$ & 62 & 66 & 74 & 80 & 96 & 208 & 23 & 469 \\
\hline $\begin{array}{l}\text { Alient T \& PI } \\
\text { Forest 40\% }\end{array}$ & 59 & 65 & 75 & 78 & 75 & 103 & 1381 & 2756 \\
\hline $\begin{array}{c}\text { Alient T \& PI } \\
\text { Forest 65\% }\end{array}$ & 56 & 66 & 73 & 79 & 66 & 163 & 869 & 3215 \\
\hline $\begin{array}{c}\text { Alient T \& PI } \\
\text { Forest 90\% }\end{array}$ & 53 & 67 & 70 & 81 & 48 & 199 & 259 & 3990 \\
\hline $\begin{array}{l}\text { Eucalyptus } \\
\text { Forest } 40 \%\end{array}$ & 61 & 65 & 75 & 80 & 67 & 101 & 1194 & 2115 \\
\hline $\begin{array}{l}\text { Eucalyptus } \\
\text { Forest } 65 \%\end{array}$ & 53 & 66 & 73 & 82 & 63 & 136 & 921 & 3947 \\
\hline $\begin{array}{l}\text { Eucalyptus } \\
\text { Forest } 90 \%\end{array}$ & 50 & 68 & 71 & 83 & 61 & 203 & 482 & 5223 \\
\hline $\begin{array}{c}\text { Koa Forest } \\
40 \%\end{array}$ & 51 & 60 & 69 & 75 & 57 & 141 & 3084 & 5020 \\
\hline $\begin{array}{c}\text { Koa Forest } \\
65 \%\end{array}$ & 48 & 62 & 66 & 76 & 49 & 180 & 2500 & 5400 \\
\hline $\begin{array}{c}\text { Koa Forest } \\
90 \%\end{array}$ & 44 & 63 & 60 & 78 & 43 & 201 & 1916 & 5935 \\
\hline $\begin{array}{c}\text { Mamane- } \\
\text { Naio Forest } \\
40 \%\end{array}$ & 37 & 47 & 51 & 64 & 34 & 47 & 5587 & 7306 \\
\hline $\begin{array}{c}\text { Mamane- } \\
\text { Naio Forest } \\
65 \%\end{array}$ & 27 & 50 & 45 & 66 & 27 & 52 & 5184 & 7992 \\
\hline $\begin{array}{c}\text { Mamane- } \\
\text { Naio Forest } \\
90 \% \\
\end{array}$ & 24 & 56 & 41 & 72 & 22 & 70 & 4564 & 8750 \\
\hline
\end{tabular}




\begin{tabular}{|c|c|c|c|c|c|c|c|c|}
\hline $\begin{array}{c}\text { Ohia Forest } \\
40 \%\end{array}$ & 53 & 60 & 70 & 77 & 70 & 148 & 2867 & 4370 \\
\hline $\begin{array}{c}\text { Ohia Forest } \\
65 \%\end{array}$ & 50 & 62 & 67 & 78 & 57 & 162 & 2336 & 4911 \\
\hline $\begin{array}{c}\text { Ohia Forest } \\
90 \%\end{array}$ & 45 & 64 & 63 & 80 & 47 & 190 & 1470 & 5787 \\
\hline
\end{tabular}

Habitat areas were identified as somewhat similar, similar, or very similar based on their climatic and elevation values. Polygons with values that fell in or between the $30^{\text {th }}$ and $70^{\text {th }}$ percentile points were deemed very similar habitat. These points were determined by identifying the median and then calculating the value above the median which would contain 20 percent of the observations, and the value below the median which would contain 20 percent of the observations. These points identified the 40 percent of the polygons that fell nearest the median and thus were identified as "very similar."

The same steps were completed to identify 65 and 90 percent of polygons nearest the median. These were identified as "similar" and "somewhat similar" respectively.

\subsubsection{Ranges Discovery Method}

After all of the datasets were aggregated, the percentile ranges associated with each vegetation type were identified using SPSS software. Three percentile ranges were identified to present the users of this model with areas of very similar habitats, similar habitats, and somewhat similar habitats for each vegetation type. Very similar habitats represented a percentile range of 40 percent centered on the median; similar habitats represented a percentile points range of 65 percent centered on the median; and somewhat similar habitats represented a percentile points range of 90 percent centered on the median. For example, the vegetation type of macadamia nuts has three percentile ranges associated with the precipitation attribute alone on Hawaii for this project. Macadamia nuts very similar habitat therefore included a precipitation range of 47 to 156 inches, a similar habitat of 46 to 194 inches, and a somewhat similar habitat of 43 to 204 inches. These percentile ranges were calculated for the temperature and elevation attributes as well. Once each vegetation type had a minimum temperature, maximum temperature, precipitation, and elevation value range for all three habitat types, the ranges were stored as SQL expressions. When the model ran, the SQL expressions then selected each polygon from the aggregated climate and elevation dataset that had each of the four specific climate and elevation attributes located within the discovered climate and elevation ranges (section 5.4).

\subsubsection{Ranges Discovery Summary}

In summary, the aggregated polygon version of the minimum temperature, maximum temperature, precipitation, and elevation were spatially compared against the vegetation types dataset. The vegetation types dataset was overlaid on top of the aggregated dataset to discover the percentile ranges of each of the four attributes located within the aggregated dataset for each specific vegetation type using SPSS software. Each specific vegetation type had three percentile ranges centered on the median of 40, 65, and 90 
percent. These three ranges were discovered to produce the habitat type ranges of very similar habitat, similar habitat, and somewhat similar habitat respectively. Minimum temperature, maximum temperature, precipitation, and elevation value ranges associated with each crop and habitat type were then stored as SQL expressions so the polygons from the aggregated climate and elevation datasets would be selected when a user chooses a specific vegetation and habitat type before running the model (section 5.4).

\subsection{Data Model Preparatory Work}

Once the aggregated dataset of minimum temperature, maximum temperature, precipitation, and elevation was overlaid on the vegetation dataset and the SQL expressions were formed, the processes to run the data model could be strung together in ArcGIS's Model Builder application. The creation of a script and its associated dialog box improved the understandability of the model, as well as improved the time to calculate the results.

\subsubsection{Vegetation Model Preparatory Work}

A model workflow was set up in ArcGIS's Model Builder application located within ArcMap, to predict the best potential locations where each vegetation type could exist (coffee, bananas, koa forests, etc.) under changes in climate. The model calculates and processes specific user defined inputs of temperature and precipitation changes to discover where the best potential areas of each vegetation type will be located. The aggregated feature class with the attributes of annual minimum temperature, annual maximum temperature, annual precipitation, and elevation was used to model the potential locations of each vegetation type. In terms of this project, many unnecessary attributes existed in the feature class's attribute table as a result of joining together four different datasets that had multiple attributes themselves. These attributes were deleted to show only the remaining attributes of "min_temp," "max_temp," "precip," and "elevation," along with the "Min_Temp_Est," "Max_Temp_Est," and "Precip_Est" mirror fields (Figure 5-9). The deletion of these unnecessary attributes also helped to reduce the amount of space that the dataset took up.

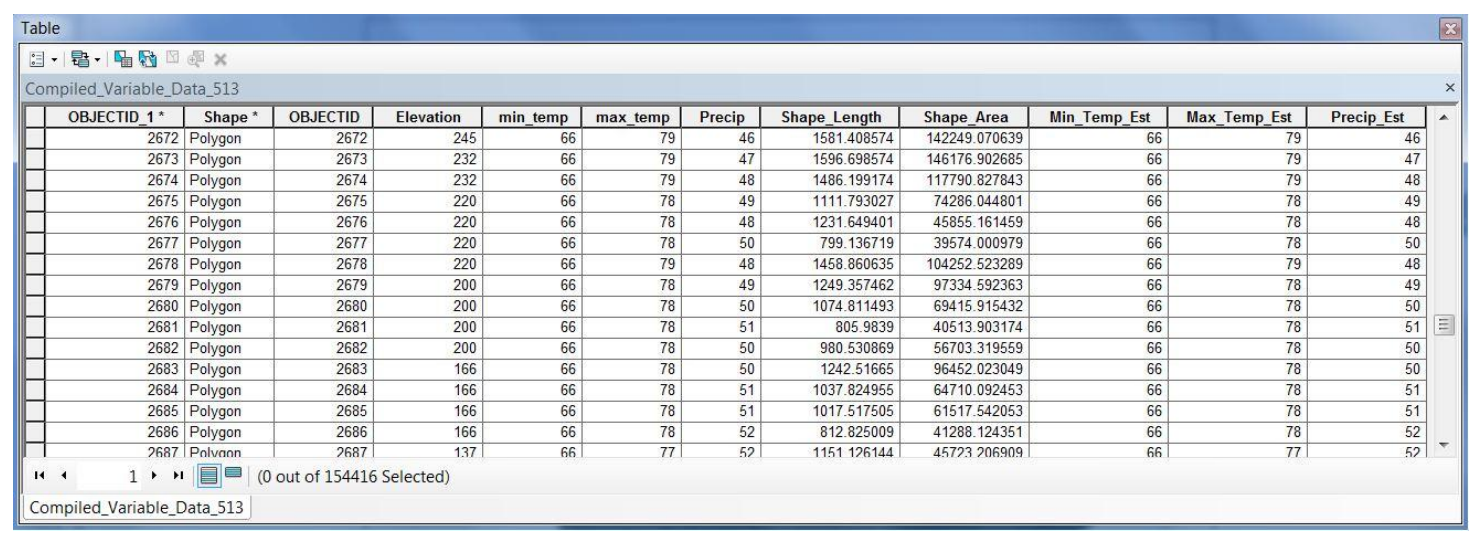

Figure 5-9: Attribute table of the aggregated dataset 


\subsubsection{Vegetation Model Construction}

Once all of the datasets were combined, the vegetation model could be created in Model Builder. The model in Model Builder allows a user to predict a minimum temperature, a maximum temperature, and a precipitation rate. The model consists of the combined datasets of minimum temperature, maximum temperature, precipitation, and elevation (called "compiled_variable_data"), three calculate field tools, a select tool, and a dissolve tool.

For the three calculate field tools and the select tool, the model called out the expression of each of the four tools and placed them as separate parameters (Appendix 1). The three calculate field tools and expressions were linked to the min_temp, max_temp, and precip attribute fields individually, so that the tools would take a predictive user input and calculate the input on these attribute fields. Important to note is that three additional fields or mirror fields (called Min_Temp_Est, Max_Temp_Est, and Precip_Est), were created in the compiled_variable_dataset to store the created values from the calculate field tool expressions. For example, the user may enter a Visual Basic for Applications (VBA) statement into the precipitation expression box, such as "[Precip] * 0.5." This VBA statement would then halve all of the values in the Precip field and store them in the Precip_Est field.

Once the values are loaded into all three of the mirror fields calculated by the user's input, the select tool is used to specify the vegetation and habitat type. In the model, the select tool uses the stored SQL expressions that were created in the ranges discovery section (section 5.3). The SQL expressions are all of the ideal minimum temperature, maximum temperature, precipitation, and elevation percentile ranges where each specific vegetation type exists under current temperatures, precipitation rates, and elevations. Therefore if the precipitation and temperature averages on the island change, the temperatures, precipitation rates, and elevations desired for each specific crop or forest type should not change and could potentially be shifted to a new area.

In the model, the user selects a habitat and vegetation type (coffee, papayas, ohia forest, etc.) by loading the SQL expressions from a folder and into the select tool. The mirror fields that calculated the user entered temperature degree change or precipitation change ratio were used by the select tool expression to select all of the polygons of the compiled_variable_dataset that fell within the percentile ranges generated for each vegetation and habitat type. For example, avocados have a 65 percent percentile precipitation range of 44 to 48 inches, centered around the median value of 46 inches. All of the polygons that have a value between 44 to 48 inches in the Precip_Est attribute field were therefore selected. This process occurs for the attribute fields of Min_Temp_Est, Max_Temp_Est, and Elevation in the compiled_variable_dataset. "AND" statements were then used in the SQL expressions so that each of the four attribute fields of Min_Temp_Est, Max_Temp_Est, Precip_Est, and Elevation all had to fall within the specified percentile ranges in one record (horizontal line in attribute table) for an individual polygon to be selected. The model workflow then dissolved the inner-polygon lines by running the dissolve tool from the Data Management Tools toolbox. Figure 5-10 displays the feature class with all of the possible polygons that could be selected and an example of a feature class with selected polygons after the modeling process have completed. 

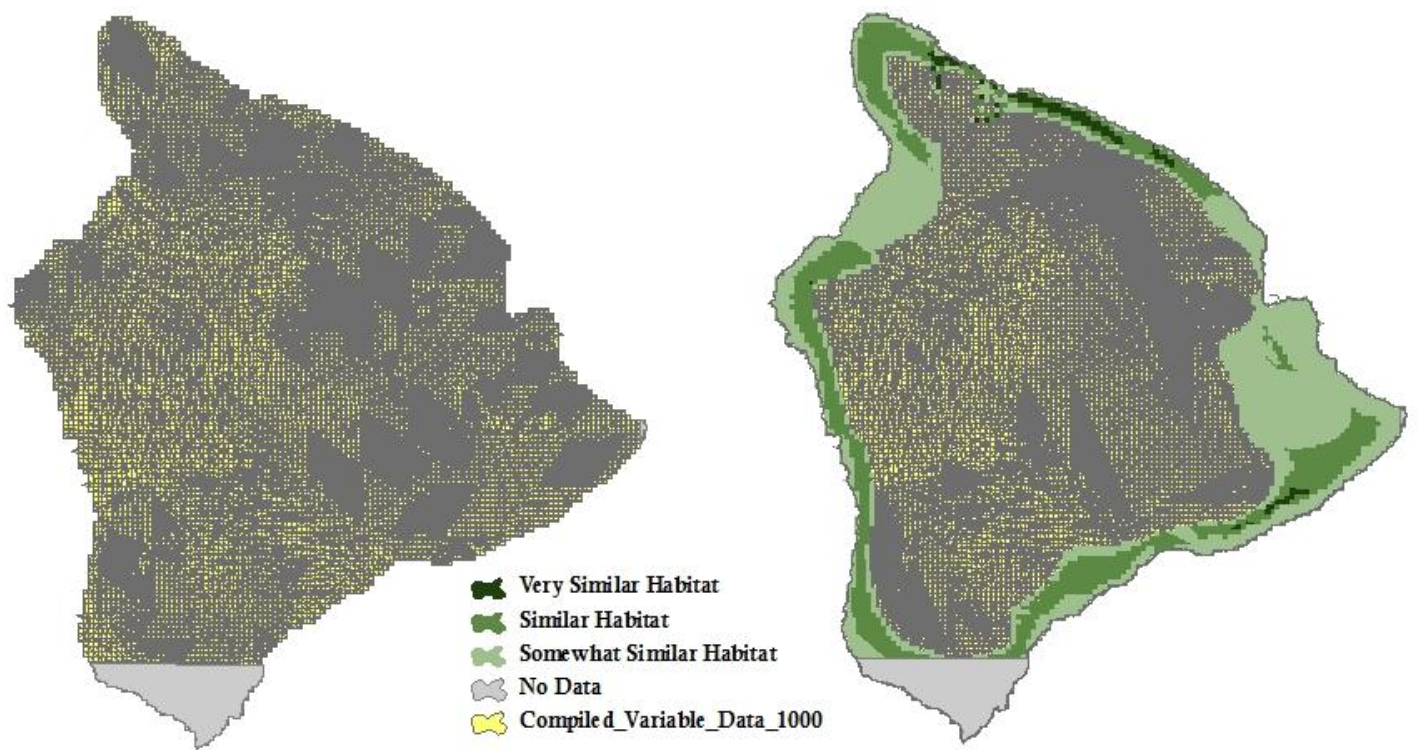

Figure 5-10: Possible polygons (left) and selected polygons (right) 


\subsubsection{Vegetation Model Script and Dialog}

The vegetation model performed the goal of predicting the potential locations of specific vegetation types when the average temperature and precipitation rates changed. However, the model's readability and processing time to run all of the tools in the model could be improved. In addition, the users could easily cause an error in the calculate field expressions by entering an invalid VBA statement. Creating a Python script (Appendix 4, Appendix 5) and dialog box (Appendix 6) to run the model improved the processing time and clarity of the inputs in the vegetation model. The vegetation script dialog box allowed the users to enter a value of 0.5 instead of the entire VBA statement of "[Precip] $* 0.5$ " to halve the precipitation values on Hawaii. Figure 5-11 visually compares the vegetation script dialog box and the vegetation model expression dialog. The scripting dialog also implemented this for the minimum and maximum temperatures. The select tool was converted into two drop down lists where the user selects the desired vegetation type in one list and the desired habitat in the other (Figure 5-12), which then brought each specific SQL expression into the scripting dialog. The user then had the option to export the created feature class to a destination folder or database of their choice. Performance wise, when running the model processes in Model Builder with the inputs of " 0 " for the minimum temperature, maximum temperature inputs, " 1 " for the precipitation input, selecting "bananas" as the vegetation type, and selecting "very_similar_habitat" for the habitat type, the processing time took 22 seconds to complete. When entering the exact same inputs into the vegetation script dialog box, the total processing time was 20 seconds, which is about 9 percent faster. The scripting dialog box also allowed instructions to be added for each input box of the script dialog box. 


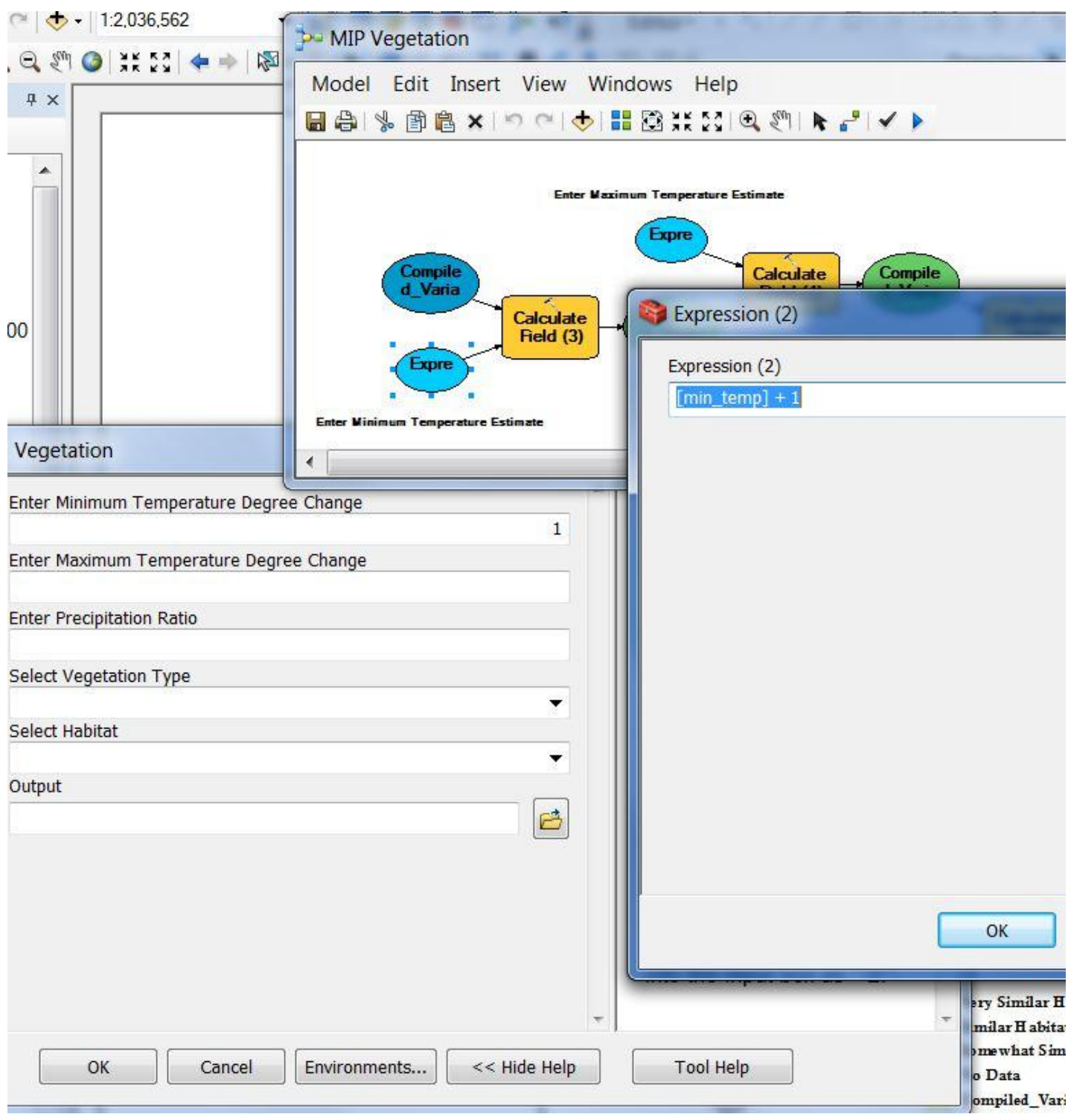

Figure 5-11: Vegetation script dialog (left) and vegetation model expression (top right) 


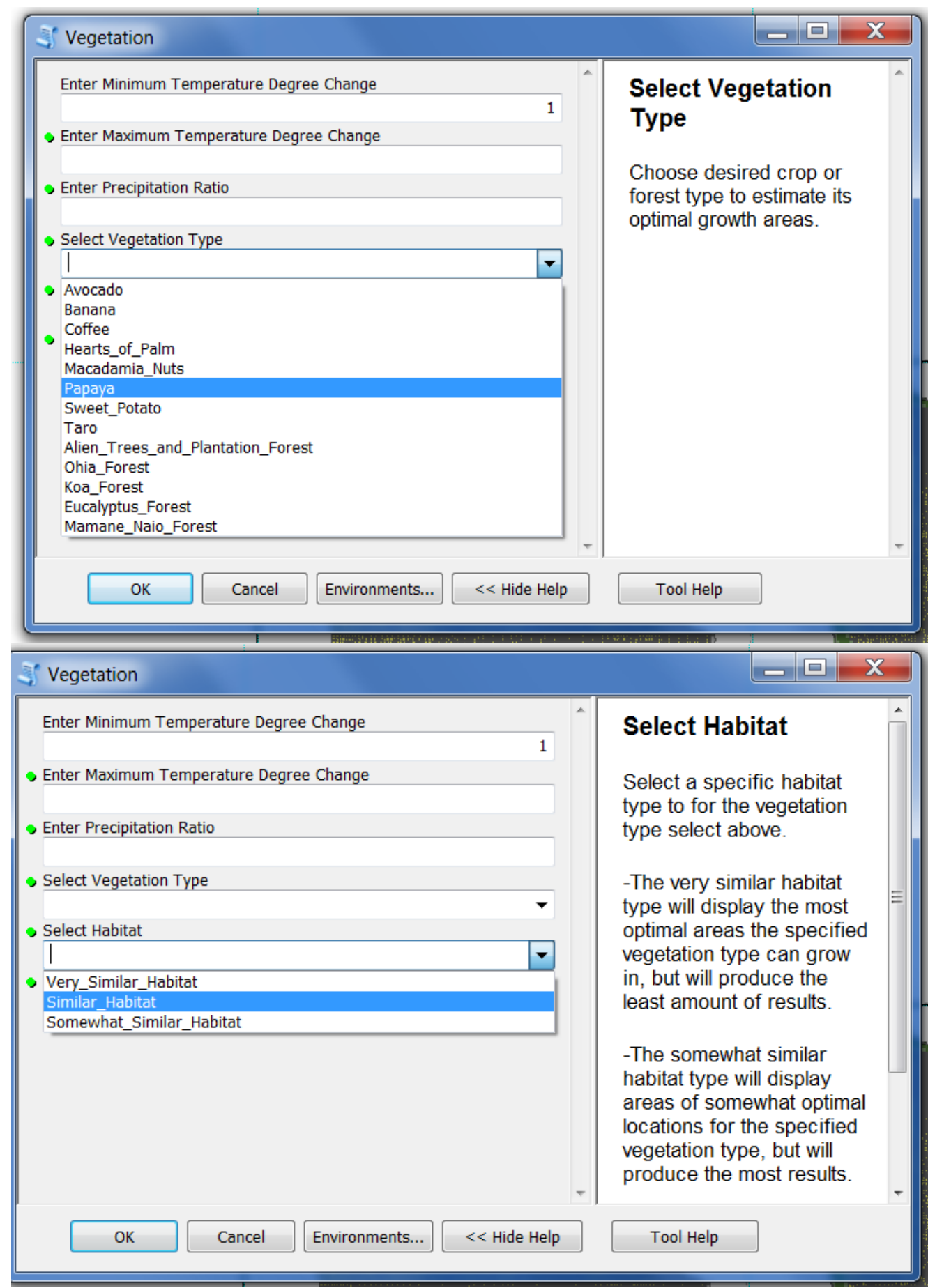

Figure 5-12: Selecting a vegetation type (top) and a habitat type (bottom)

\subsubsection{Data Model Preparatory Work Summary}

The vegetation model was created using ArcGIS's Model Builder application. The model utilized the aggregated polygon datasets of minimum temperature, maximum 
temperature, precipitation, and elevation to produce one large aggregated dataset referred to as "compiled_variable_data." A user-specified SQL expression (representing a vegetation and habitat type) then selected any polygons from the compiled_variable_data that fell within the climate and elevation percentile ranges defined by the SQL expression. The best potential locations of each of the vegetation types were identified, after the dissolve tool eliminated the intermediary boundaries. Finally, a Python script was used to convert the model into a dialog box to simplify user interaction.

\subsection{Comparison of Interpolation Algorithms}

The Hawaiian Vegetation Model project interpolated the three surfaces of minimum temperature, maximum temperature, and precipitation using two interpolation algorithms - ordinary kriging and inverse distance weighted (IDW) (Figure 5-13). Figure 5-14 displays the semi-variogram and inputs used to generate the minimum temperature surface. Figure 5-15 displays the semi-variogram and inputs used to generate the maximum temperature surface. Figure 5-16 displays the semi-variogram and inputs used to generate the precipitation surface. The very uneven distribution of the data points warranted the more complex kriging algorithm, and the resulting surfaces appeared more realistic. The kriging routine had the additional advantage of generating uncertainty surfaces for each dataset (Figure 5-17). In Figure 5-17, the lightest color represents the areas with the lowest error, and the darkest red color represents the areas with the highest uncertainty. The maps show that the areas with highest uncertainty are located the furthest away from any rain gauge stations. This occurs in the inland areas on the western side of the island for the minimum and maximum temperature error surface maps as well as the inland areas and the southeastern coast for the precipitation error surface map. 


\section{IDW}

Kriging

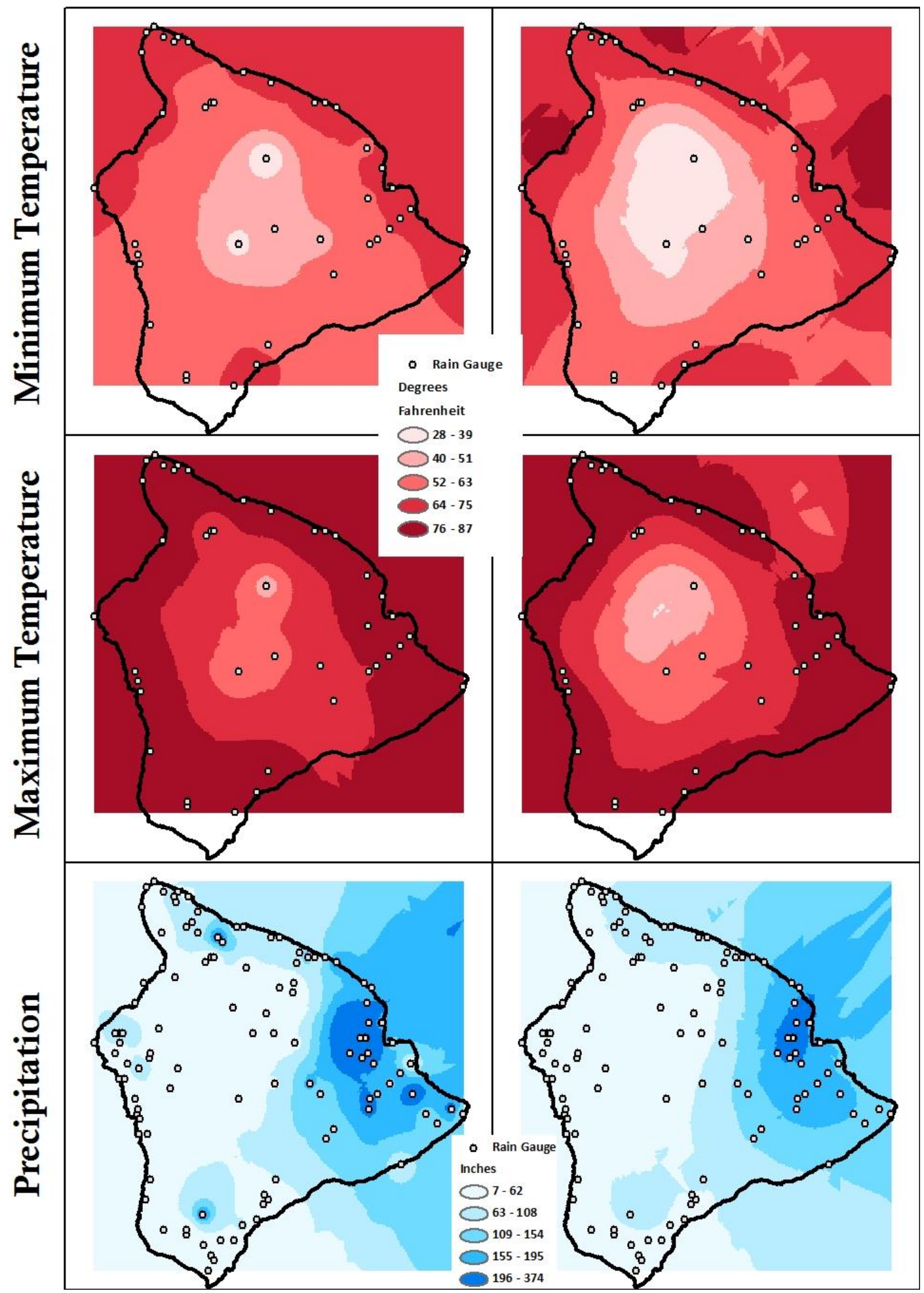

Figure 5-13: Comparison of IDW and Kriging Surfaces 

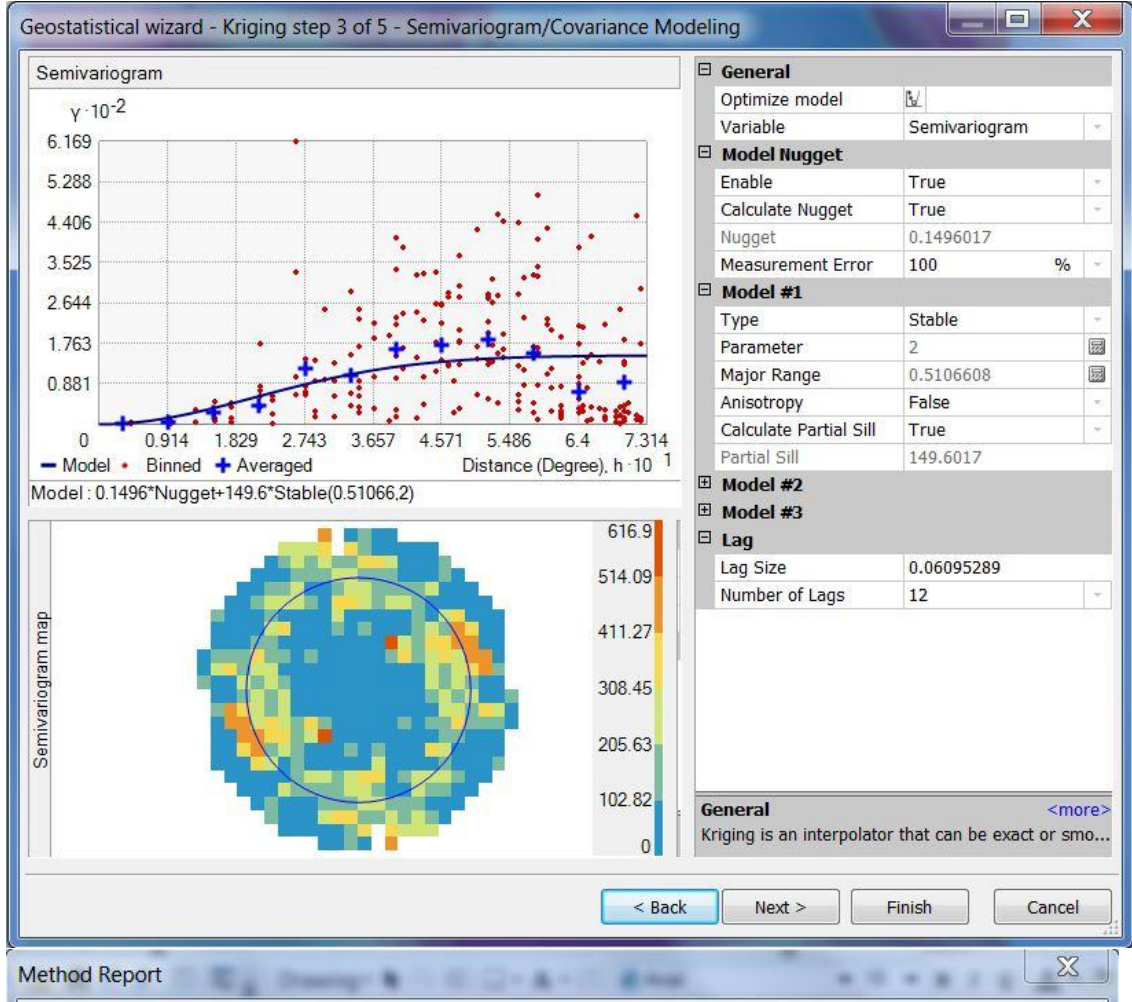

\section{Input datasets}

$\boxminus$ Dataset

Acc_Temp_Only

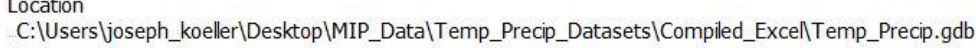
Type

Records 41

$\boxminus$ Method Kriging

Type Ordinary

Output type Prediction

$\boxminus$ Dataset \#

Trend type

Searching neighborhood

Type

Neighbors to include $\quad 5$

Include at least $\quad$ Four and 45 degree

$\begin{array}{lr}\text { Sector type } & \text { Four and } 45 \text { degree } \\ \text { Angle } & 0\end{array}$

$\begin{array}{ll}\text { Major semiaxis } & 0.5106608206247637\end{array}$

Minor semiaxis $\quad 0.5106608206247637$

$\boxminus$ Variogram Semivariogram

Number of lags 12

$\begin{array}{lr}\text { Lag size } & 0.06095289407903772\end{array}$

Nugget $\quad 0.14960171803363762$

Measurement error \% 100

ShiftON No

घModel type Stable

Parameter 2

$\begin{array}{ll}\text { Range } & 0.5106608206247637\end{array}$

Anisotropy No

$\begin{array}{lr}\text { Partial sill } & 149.6017180336376\end{array}$

Save...

Cancel

Figure 5-14: Minimum Temperature Interpolation Inputs 


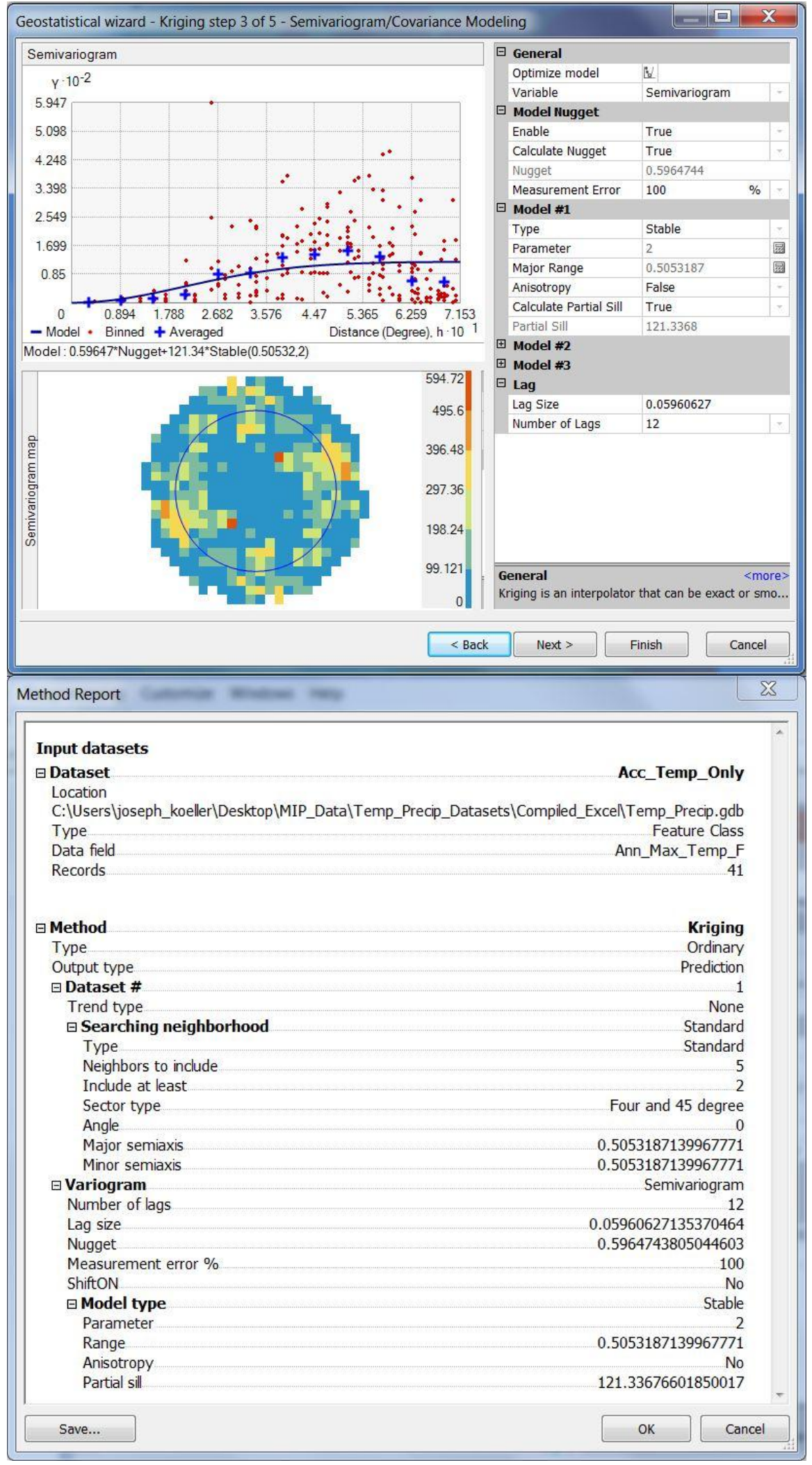

Figure 5-15: Maximum Temperature Interpolation Inputs 


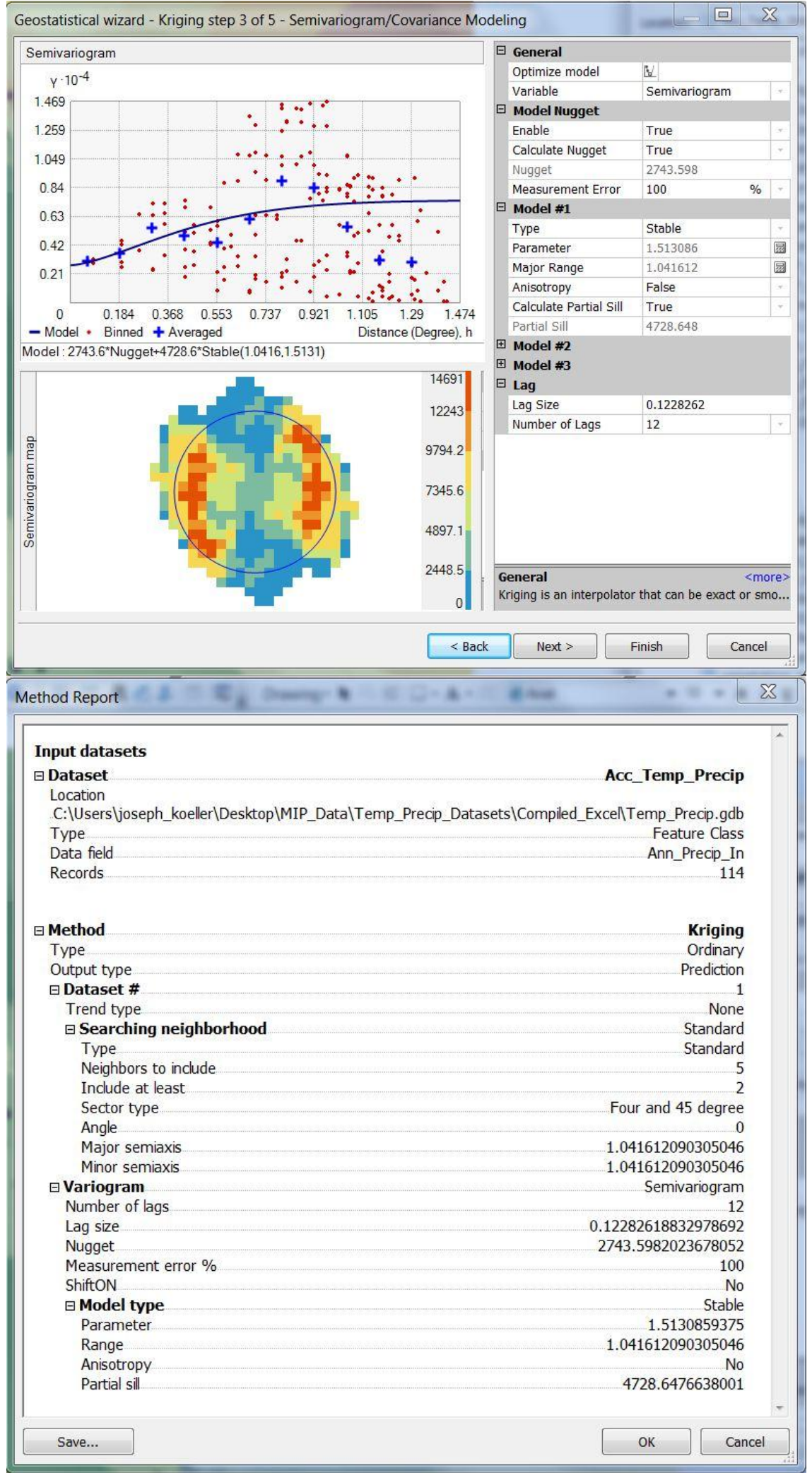

Figure 5-16: Precipitation Interpolation Inputs 


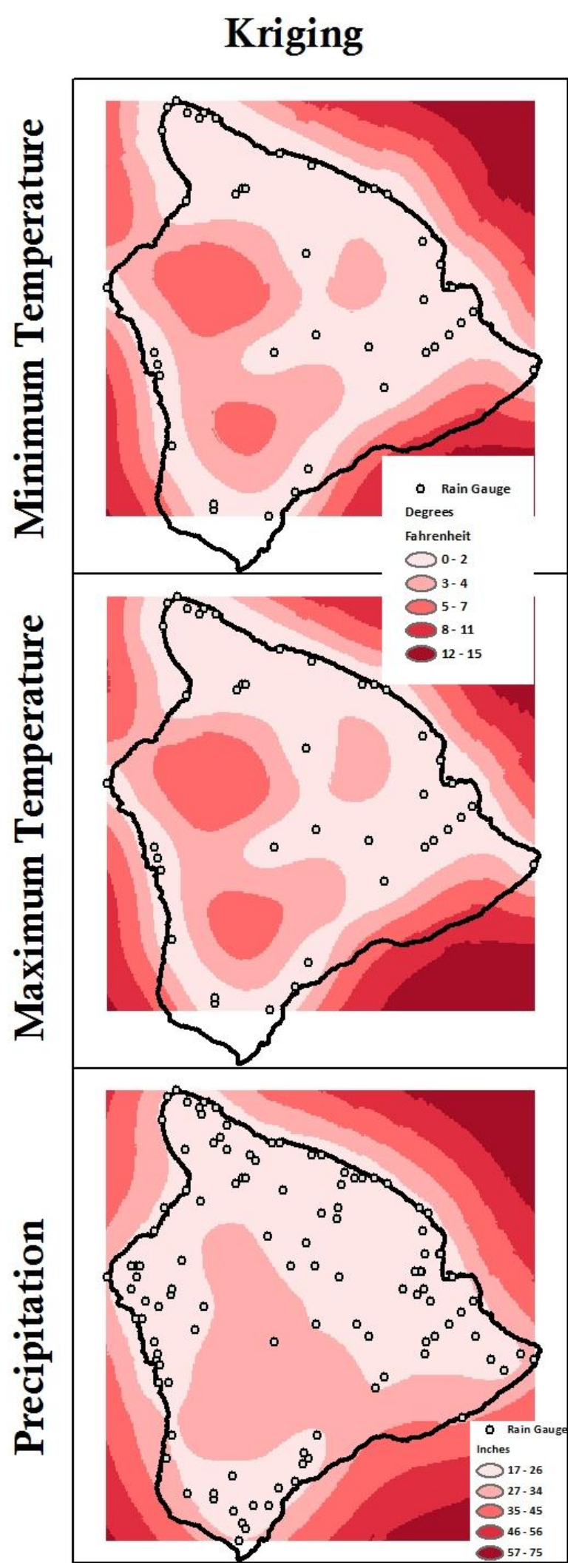

Figure 5-17: Error Surfaces for Each Dataset 


\subsection{Summary}

After the project data were downloaded, the vegetation model could be created. In order to create the model, further preparatory work was completed on the temperature, precipitation, elevation, and vegetation land use datasets to specifically modify them for subsequent model processes. Once the preparatory work was complete, percentile ranges of temperature, precipitation, and elevation were discovered for the each of the specific crop and forest types (for example, coffee exists in the 90 percent percentile points precipitation range of 44 to 60 inches centered around the median). Once the datasets were specifically modified for the model, it was created by inserting the modified datasets, geoprocessing tools, and the tool expressions.

Modification and preparatory work performed on the datasets was necessary before the datasets could be incorporated into the vegetation model. The temperature and precipitation datasets had to be interpolated into a raster surface from their previous vector surfaces. Polygons were created from each of the three created raster surfaces. The elevation dataset originally came as a rasterized DEM, which was then resampled into a 250-meter DEM and a 1000-meter DEM. The resampled 250-meter DEM was used to discover the climate and elevation ranges for each specific vegetation type. The resampled 1000-meter DEM was used to reduce the processing and drawing times of the model. All four datasets (minimum temperature, maximum temperature, precipitation, and elevation) were converted into a polygons so they could be merged together.

Once the temperature and precipitation point datasets were converted to rasters and then to polygons, and the resampled 250-meter raster DEM was converted to polygons, the temperature, precipitation, and elevation ranges for each specific crop and forest type could be identified and implemented into the model as SQL expressions. First, the select by location and select by attributes tools were used in conjunction to overlay the vegetation dataset onto the aggregated dataset of the minimum temperature, maximum temperature, precipitation, and elevation. Once overlaid, each polygon from the aggregated climate and elevation dataset that intersected with a specific vegetation type was selected. The selected polygons of the aggregate dataset were then exported to SPSS for statistical analysis. The SPSS statistical software package was used to identify the percentile ranges for each vegetation type. Areas with 40, 65, and 90 percent percentile ranges around the median were used to identify areas with very similar, similar, and somewhat similar values.

After the ranges discovery step was complete, the vegetation model could be constructed. For the vegetation model, the minimum temperature, maximum temperature, precipitation, and the resampled 1000-meter DEM vector polygon datasets were combined into one large dataset, compiled_variable_data, using the identity tool. The model was created using the compiled_variable_data feature class, as well as three calculate field tools, a select tool, a dissolve tool, three VBA expression boxes connected to the calculate field tools, and one SQL expression box connected to the select tool.

Python scripts were created to produce dialog boxes that allow the user to enter values for the possible change. The model then runs and identifies areas with suitable habitats after the climate changes. 



\section{Chapter 6 - Results and Analysis}

\subsection{What Are the Users Allowed to do with these Models?}

Once the vegetation model was completed, the users of the model enter their own climate estimates to determine the potential locations of various vegetation types on Hawaii. The created script and associated dialog box allow the users to enter an estimated ratio of precipitation and degree changes of minimum and maximum temperature to predict how the climate could change in the future. From a drop down menu, the user also chooses a specific vegetation and habitat type which represents an SQL statement. The audiences for this project are county planners interested in predictive vegetation type research on Hawaii. The scripting dialog (Appendix 6) provides the county planners with an efficient way to determine where the potential locations of specific vegetation types could grow, if the temperature and precipitation averages change in the future.

The script allows input of what the user thinks the minimum temperature, maximum temperature, and precipitation rates will be in the future. These entered estimates are in ratio form for the precipitation input, and in a degree Fahrenheit change for the temperatures input. For example, if the user enters the numeric value of 1 in the precipitation estimate box, then the resulting potential locations will reflect the possible areas where that specific crop could grow under the current precipitation condition. If the user enters the numeric value of 2, then the resulting potential locations will reflect the possible areas of where the chosen vegetation type could grow if the current precipitation conditions doubled. If the user enters a numeric value of 0.5 , then the resulting potential locations reflect the possible areas of where the chosen vegetation type could grow if the user halved the current precipitation rates. ArcGIS tools simply multiply the ratio that the user enters by the current precipitation averages to reflect the potential change of precipitation rates in that area and adds/subtracts degree changes to the current temperature averages to reflect the potential change of temperatures.

Once the user enters the ratios and the degree changes, they select a vegetation and habitat type to apply the specified estimates. Since the model selects climatic polygons from a feature class, the vegetation and habitat types are in the form of SQL statements that specify which polygons to select when running the model/script. The user selects a vegetation and habitat type by loading the saved SQL queries for each vegetation and habitat type by picking a vegetation and habitat type separately from a drop down menu in the vegetation script. Once the user enters the temperature degree changes and the precipitation ratio, along with the chosen vegetation and habitat type, they can specify where to save the feature class before the model's processes run.

The vegetation model was created to provide county planners with a predictive vegetative analysis that they would otherwise not have, or would have to perform themselves. The creation of the scripting dialog provided the county planners with a more efficient tool to use than otherwise running the model through ArcGIS's Model Builder. The model shows the ranges of the potential locations where a specific vegetation type grows if certain climatic changes occur. The model did not take into effect the current areas of any urbanization or preservation, because any potential changes could happen to that area in the future, such as urbanization in current farmlands or new farmlands in a 
currently urbanized area. County planners could potentially use this model to manage where specific crop types are potentially grown in the future and perhaps preserve those areas in case their estimated temperature and precipitation scenarios happen.

In summary, the vegetation model of this project provided the user audience of county planners and efficient tool to use for performing predictive vegetative analysis with changing climatic inputs. The script dialog box consisted of entering a ratio for the precipitation rate input and degree change inputs for a minimum temperature and a maximum temperature for climatic analysis, in addition to selecting a vegetation and habitat type. The dialog box processes the models' functions to show all of the potential locations of the vegetation type based on what the user enters as the climatic inputs. Finally, the county planners who use the scripting dialog can determine the future potential placement of specific vegetation types, and perhaps preserve that area specifically for that crop or forest type in case the temperature and precipitation averages do change.

\subsection{What Happens When the User Runs the Models?}

The vegetation model of this project was demonstrated twice to show the results of a specific crop type in the first scenario and a specific forest type in the second scenario. Coffee was chosen as the demonstrative variable among the crop types for the first scenario. Ohia forests were chosen as the demonstrative variable among the vegetation types for the second scenario.

The coffee crop type demonstrates the varying potential locations of one of eight crop types in the Hawaiian Vegetation Model project. The actual areas of coffee crop plantations lie on the western side of the island (Figure 6-1). In previous sections in this paper, the western side of the island was determined to be much drier compared to the eastern half. The actual coffee plantations also appear to be closer to the coast, which means the temperatures are higher, and the elevations are lower when compared to the rest of the island. From the gathered climatic data of the actual coffee plantations, the potential areas for coffee cultivation on the island under the current conditions is for the most part where most of the actual coffee locations are located currently as well as new potential areas in the northern and southern tips of the island (Figure 6-2). If performing the predictive analysis under the scenario of increased precipitation rates of 30 percent and increased minimum and maximum temperatures of three-degrees Fahrenheit, the potential locations of coffee shift to the north and south of the actual coffee plantations, while still being located in proximity to the coast (Figure 6-3). From the generated scenarios in this section, it is evident that under increasing average temperatures and precipitation rates, the possible areas to grow coffee shrink. Looking at a scenario with reduced temperatures and precipitation rates, such as a 10 percent decrease in precipitation and a three-degree decrease in average minimum and maximum temperatures, the potential locations of the coffee areas have again changed from the potential coffee areas under current conditions. More specifically, the potential locations to grow coffee exist in a large area on the southern end of the island, two areas in the northern edge of the island, and another large area just north of the existing coffee plantations (Figure 6-4). In addition to the climate scenario examples mentioned above, the user can choose from any combination of minimum temperature, maximum 
temperature, and precipitation rates to determine the areas of a vegetation type that they may desire.

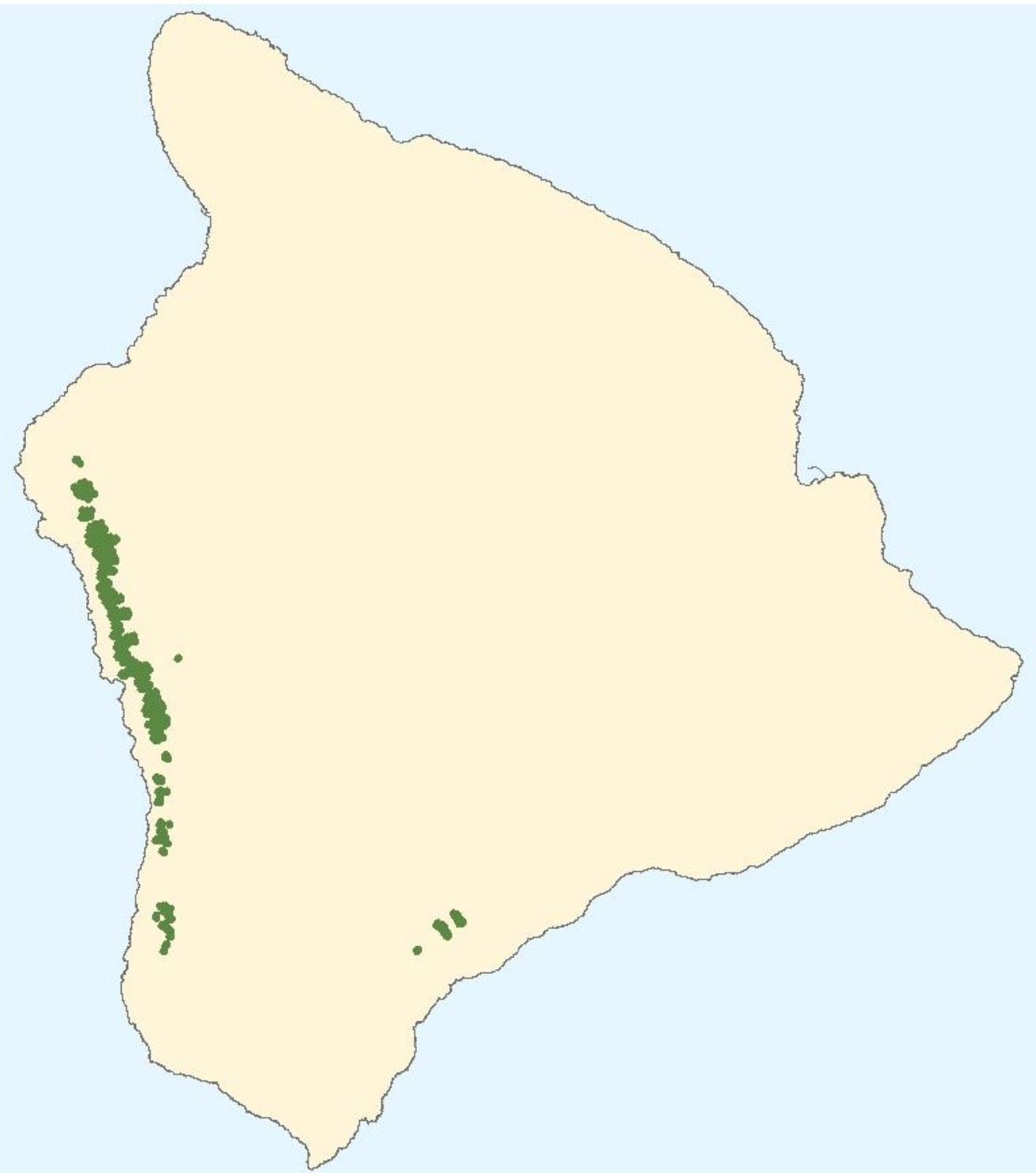

Figure 6-1: Located coffee plantations 


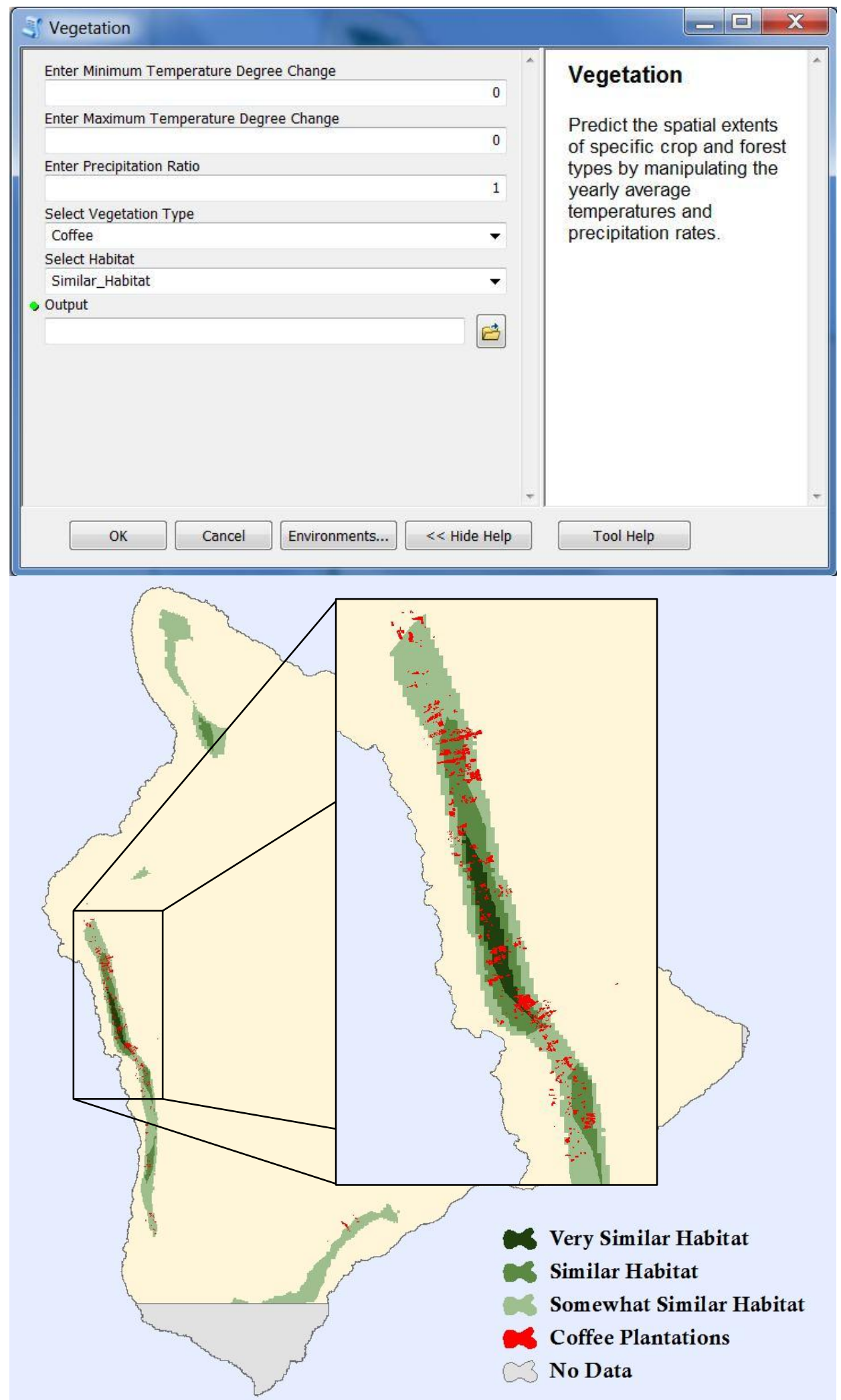

Figure 6-2: Potential coffee locations under current climate conditions 


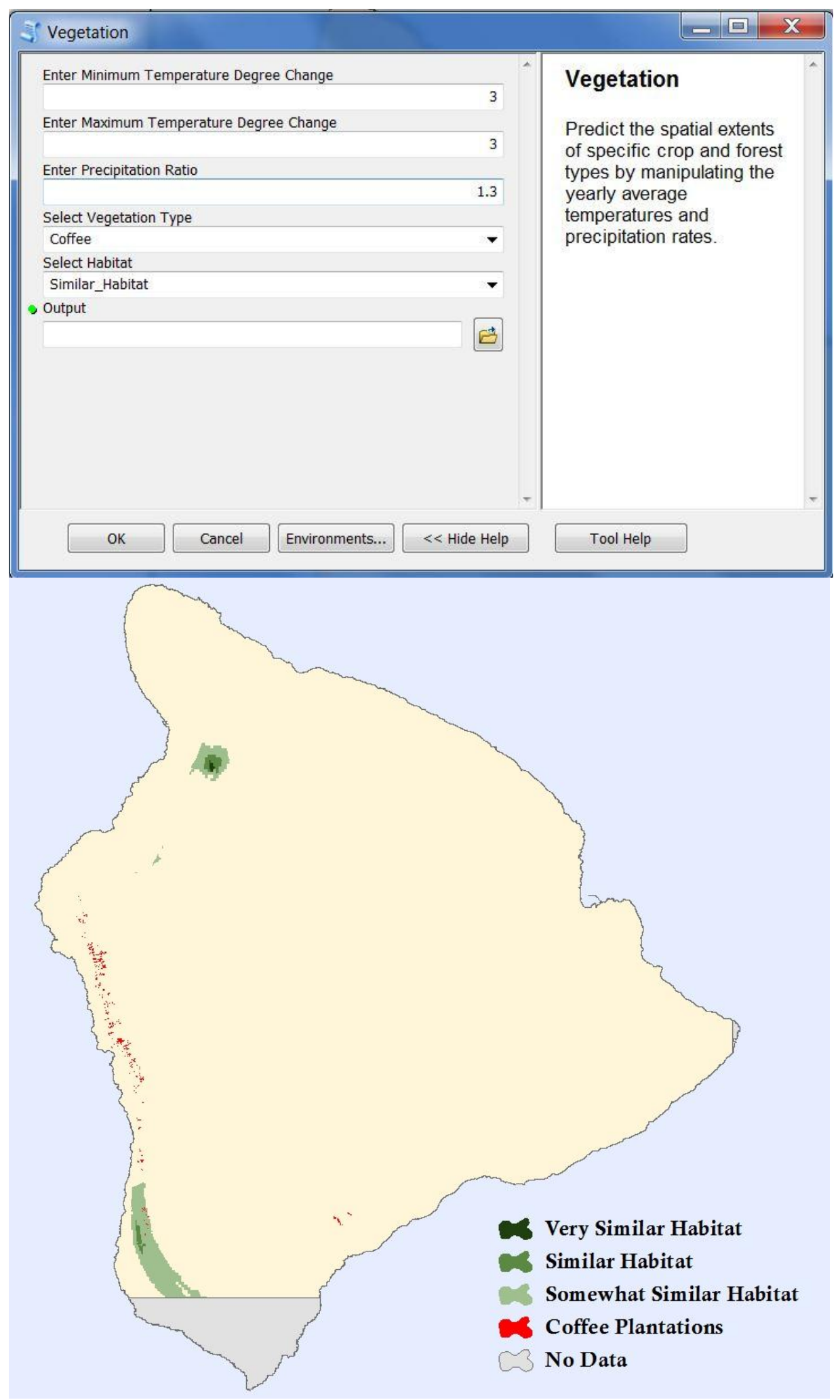

Figure 6-3: Potential coffee locations under climate increases 

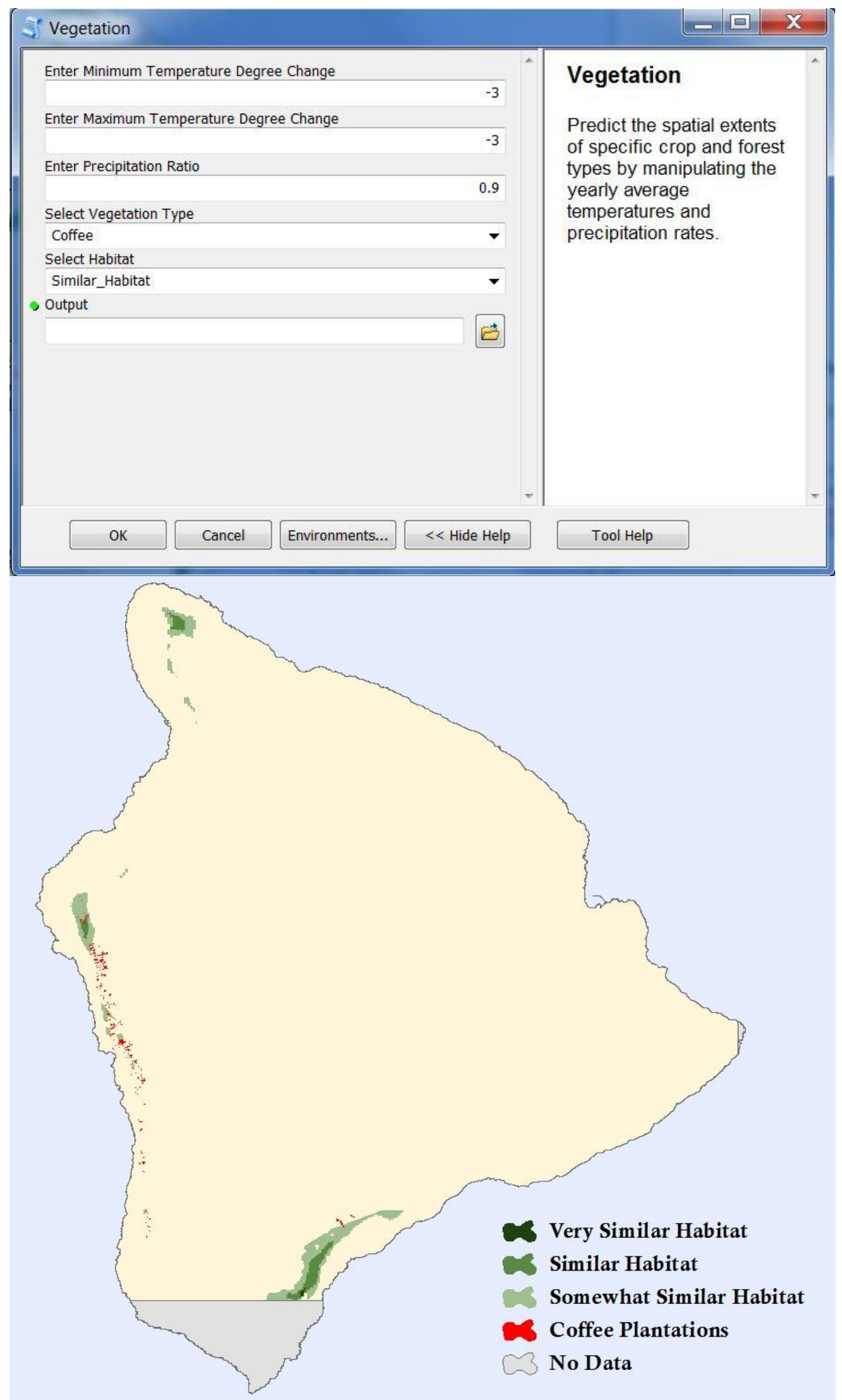

Figure 6-4: Potential coffee locations under climate decreases 
If a user chooses to use the ArcGIS Explorer version of the created model as opposed to the ArcGIS/ArcMap version, the user enters temperature and precipitation inputs through a Python executable (.exe) file and views the results in ArcGIS Explorer. Figure 6-5 demonstrates an example of the coffee crop type under current climate conditions with the entered values in the Python executable file. Notice that the results are exactly the same as Figure 6-2, but with different symbology (limited in ArcGIS Explorer compared to ArcMap).

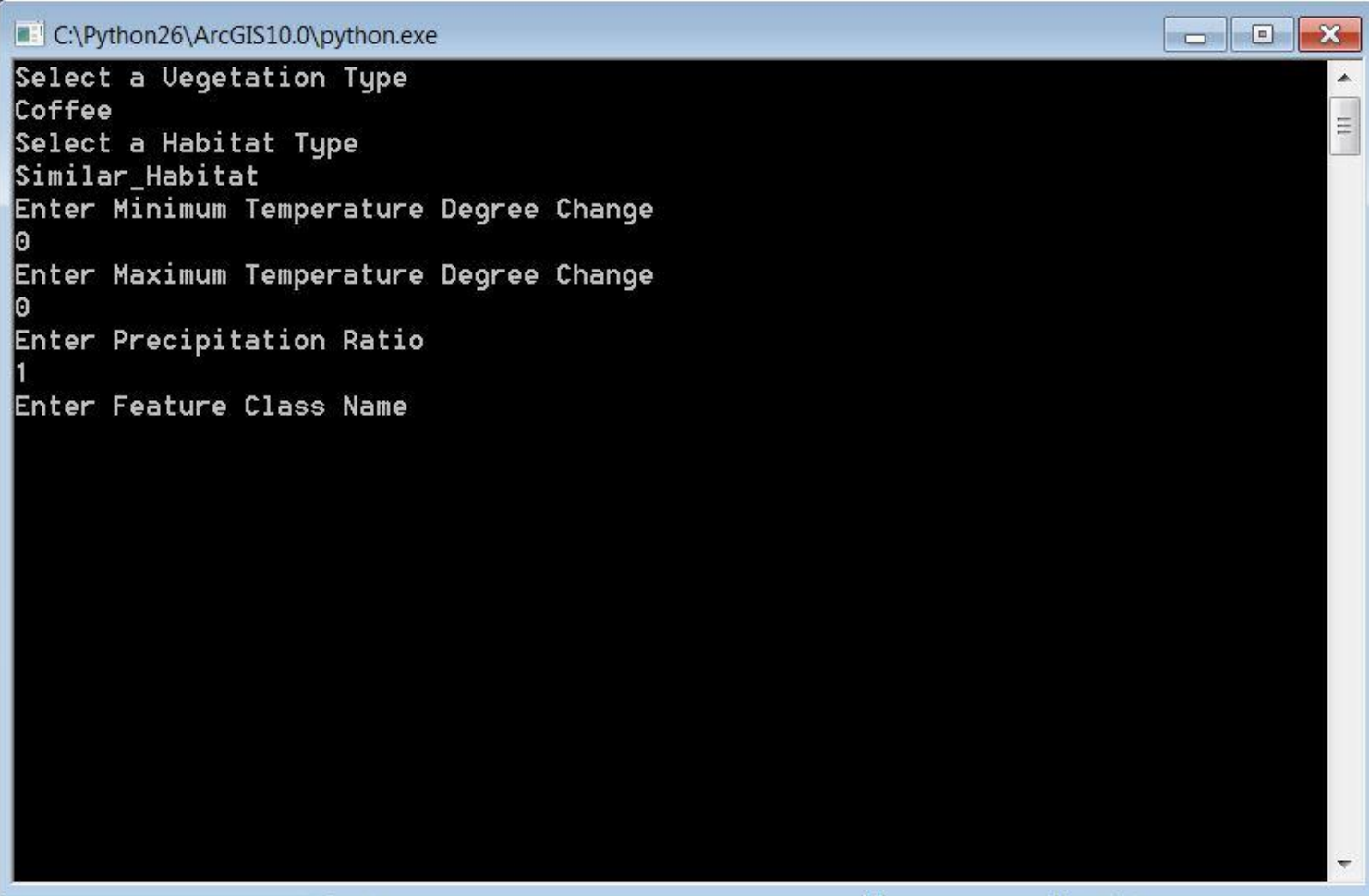




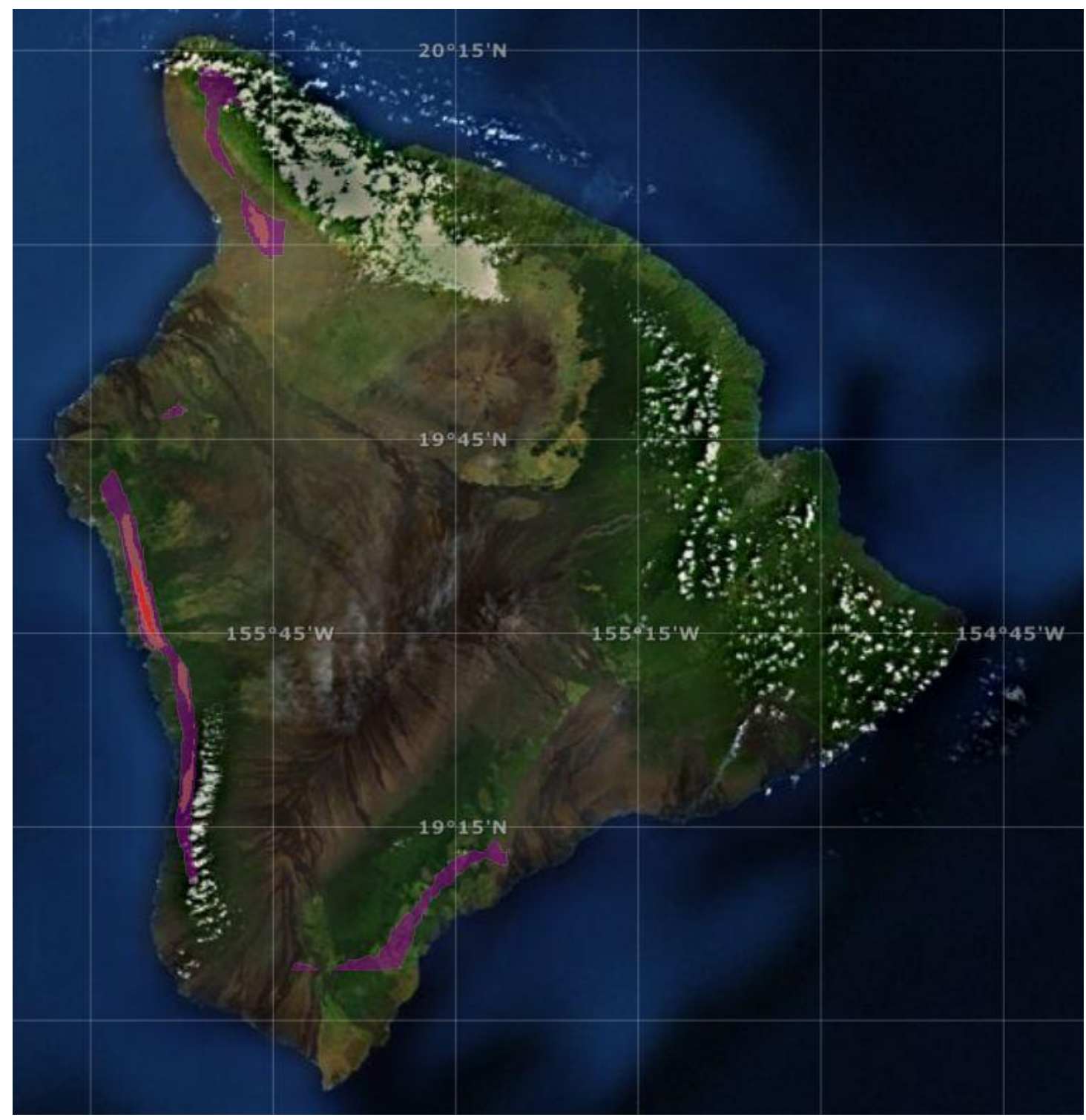

Figure 6-5: ArcGIS Explorer Results

Turning to a predictive landscape analysis of a forest attribute, as opposed to a crop attribute, the project tested the ohia forest type as one of the five forest types available. The original ohia forests are disbursed quite evenly across the island, when looking at the actual areas (Figure 6-6). When calculating the potential locations of the ohia forests under current temperature and precipitation conditions, a wide area all around island is covered with the exception of a large area in the center of the island (excluding the no data zone on the southern tip) (Figure 6-7). Using a scenario of increased temperature and precipitation rates to predict the potential locations of the ohia forests under these conditions, this example tested a 50 percent increase in precipitation and a five-degree increase in minimum and maximum temperatures. The result shows the potential location of the forest shrinking to the center of the island, most likely because of the temperature increases (Figure 6-8). Using a scenario of decreased temperatures and 
precipitation rates to predict the potential location of the ohia forests under these conditions, this example tested a 50 percent decrease in precipitation rates and a fivedegree decrease in minimum and maximum temperatures. The result shows the forest locations shifting to the easternmost (wetter) half of the island, probably because the western half of the island is too dry for the forest to flourish under the specified scenario (Figure 6-9).

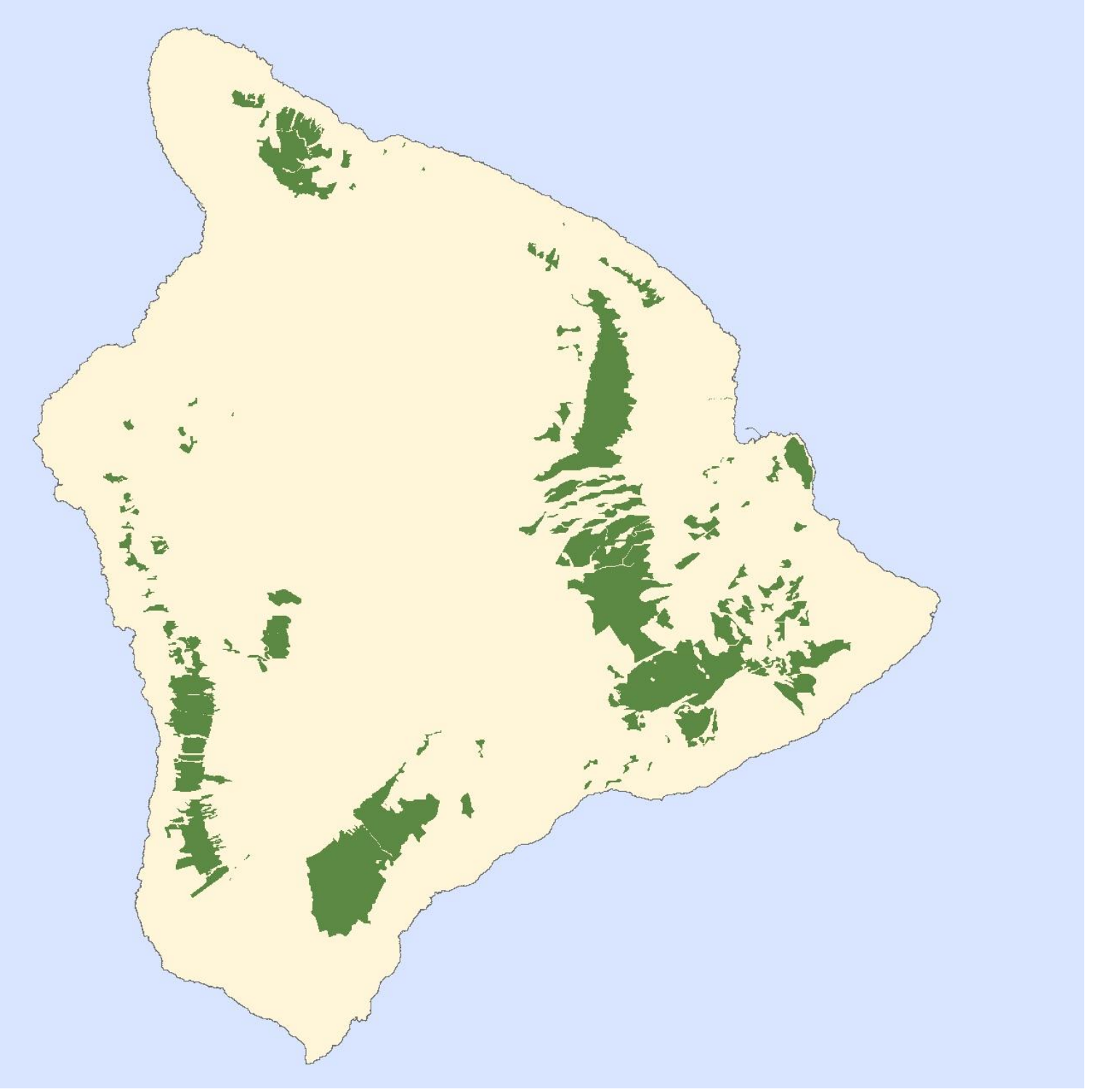

Figure 6-6: Ohia forests 

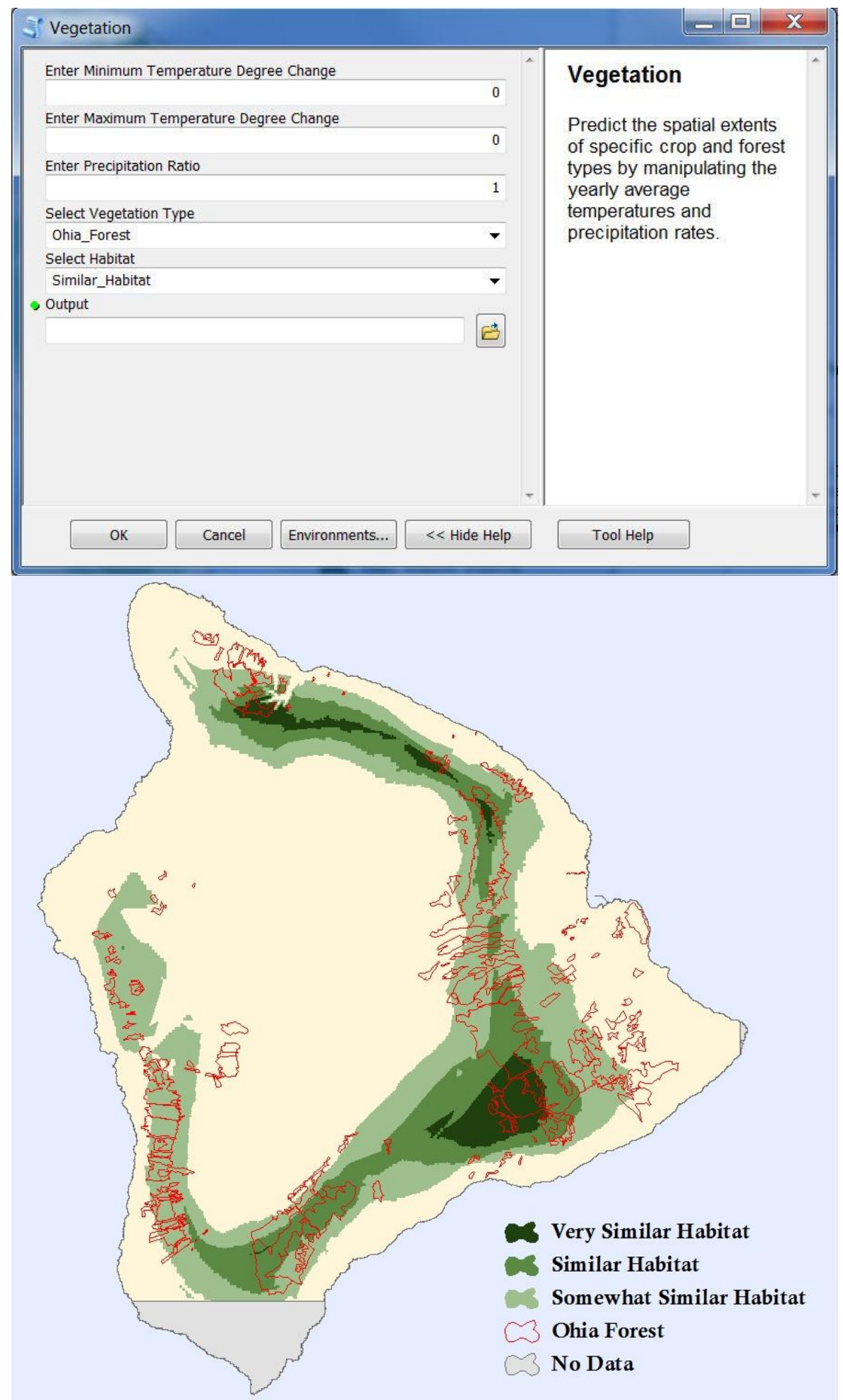

Figure 6-7: Potential ohia forest locations under current climate conditions 




Figure 6-8: Potential ohia forest locations under climate increases 


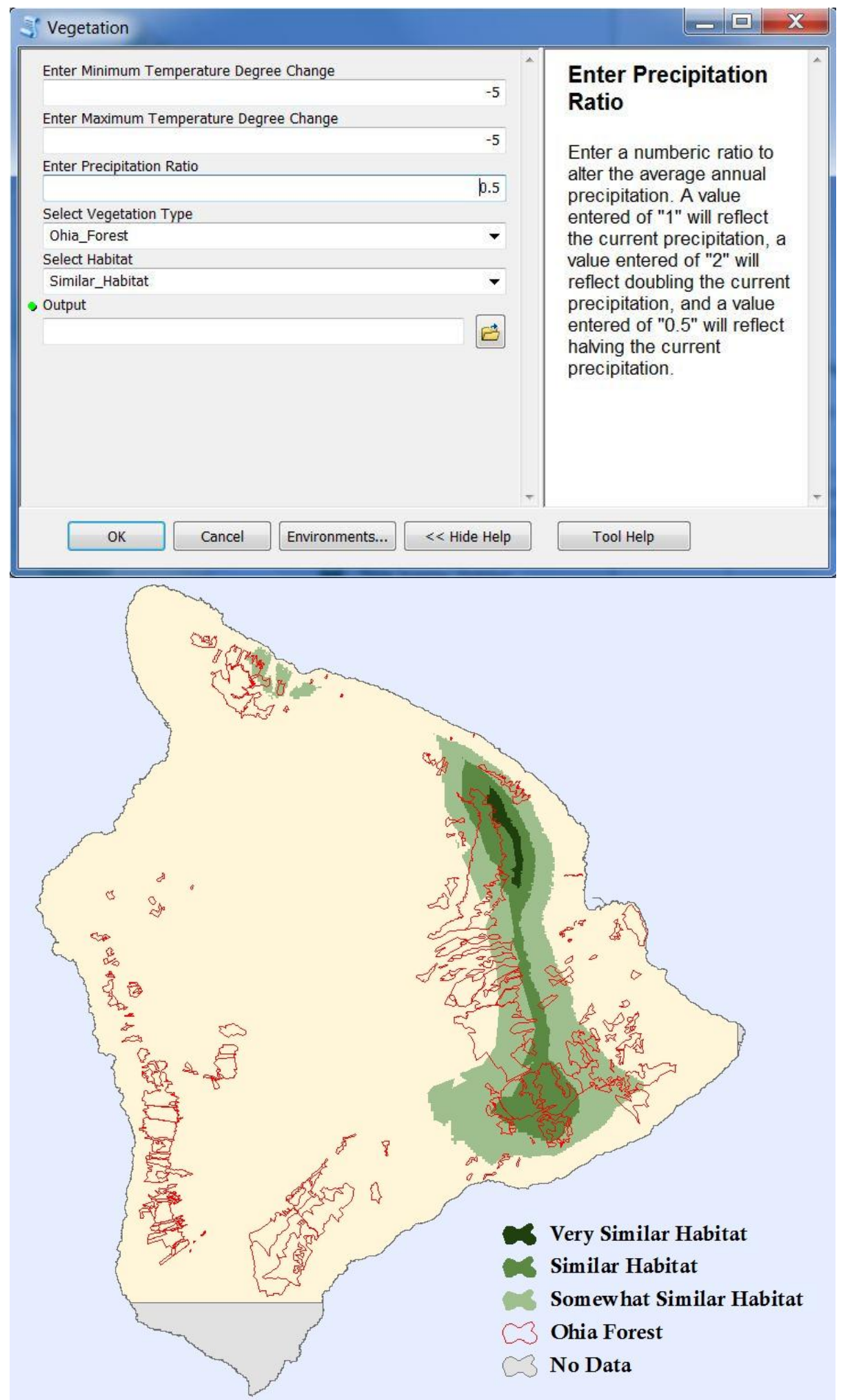

Figure 6-9: Potential ohia forest locations under climate decreases 
When looking at the figures produced for the potential areas of ohia forests, it is noticeable that the majority of the forests do not match up with the best potential areas under the current climate conditions. This is due to the bi-modal distribution of data in the precipitation attribute (Figure 6-10). The bi-modal distribution is causing the median and percentile range of the data to occur where the amount of records of ohia forest are not as well represented as other precipitation (percentile) ranges as shown in Figure 6-10. For example, Figure 6-11 shows the 65 percent percentile points results of each attribute mapped out individually, with the 65 percent percentile points range shown with a black outline. Notice that the precipitation (bottom left) graphic does not match up with the ohia forests as most of the forests on the western side of the island fall outside of the potential precipitation area. Therefore, the frequency distributions of the attributes need to be looked at before producing any results.

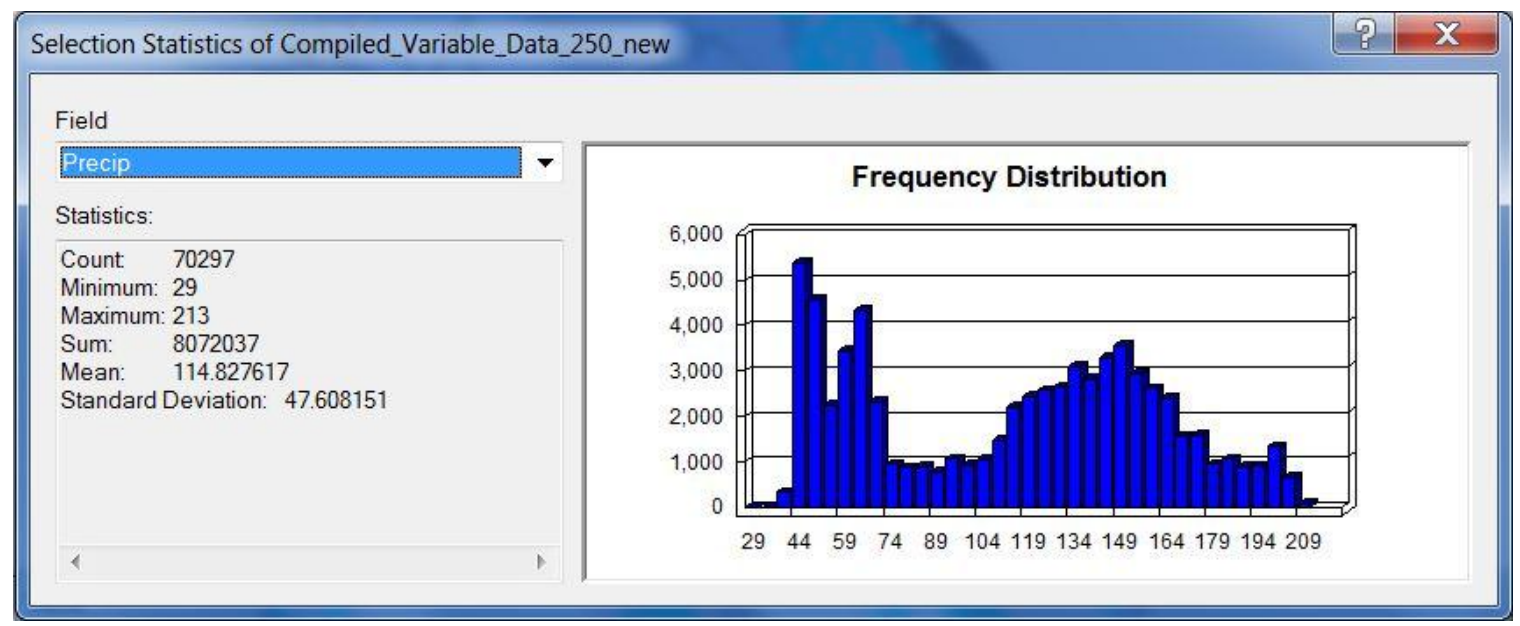

Figure 6-10: Ohia Forest Precipitation Attribute Distribution 


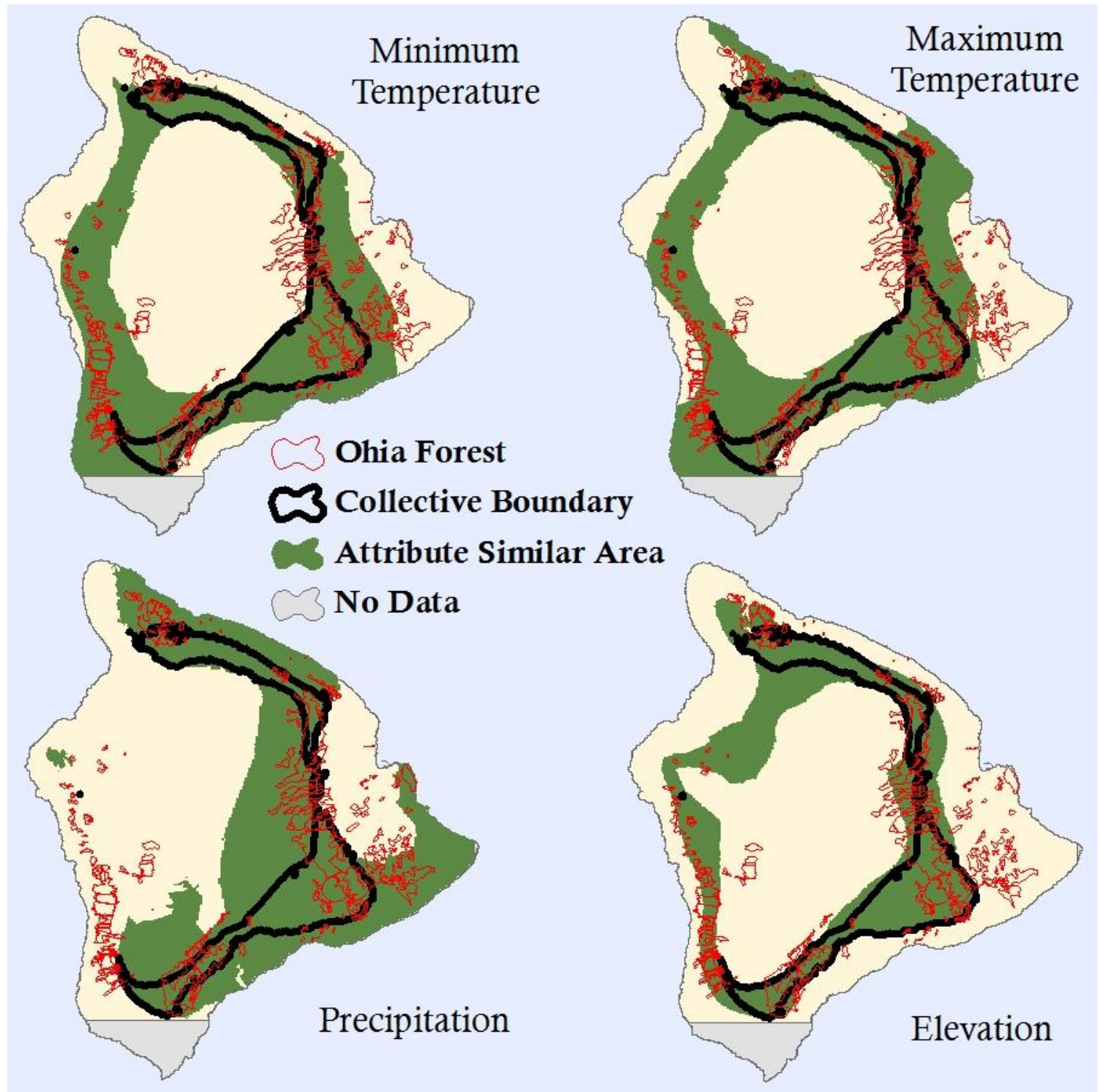

Figure 6-11: Potential areas for each of the four attributes individually

In summary, this section previewed how county planners could potentially run the vegetation model. For the model, the potential locations of the coffee crop type were calculated under the scenarios of increased and decreased precipitation and temperature. The potential locations of the ohia forest under the forest type attribute were also calculated under the scenarios of increased and decreased precipitation and temperatures. These scenarios showed how the potential locations of specific vegetation types could possibly change when yearly temperature and precipitation averages change.

\subsection{What Process Went Right? What Process Went Wrong?}

Throughout the process of creating the vegetation model, certain aspects went according to plan, while others did not work out as well. In terms of creating the model and creating 
a tool that predicts the possible locations of specific vegetation types when temperature and precipitation averages change, this project was a success. However, poorly represented vegetation types (compared with the other vegetation types) in terms of the number of records/plantations in the vegetation land use dataset, offer little predictive analysis, such as the hearts of palm crop type.

Creating a successful script that inputs the county developer's temperature and precipitation scenarios went according to plan in this project. The script's dialog box requires the user to enter temperature degree changes, a precipitation ratio, a vegetation type, and a habitat type to run the rest of the processes. This allows the county planners to make their own estimations of how the climate might change in the future; they can then weigh the results of the produced potential locations of the vegetation type selected.

In the model, the prediction of potential locations of certain vegetation types is more accurate than others because some vegetation type records were not as well represented as others when observing the attributes of the original vegetation land use dataset. This is due to the original dataset not locating enough areas across Hawaii in the remote sensing process (if there actually are any more) where certain vegetation types could potentially exist. For example, remote sensing techniques located hearts of palm plantations in one specific area on Hawaii's northeastern end (Figure 6-12). As a result, the plantations did not have a wide enough spatial distribution across the island to catch a much wider range of temperature, precipitation, and elevation values, so its potential growth area under current conditions is also hampered (Figure 6-13). This example is still included in the project, because more than 30 polygons were selected when overlaying the hearts of palm crop type on the aggregated climate and elevation dataset. Any vegetation type that selected at least 30 polygons in the aggregated climate and elevation dataset were kept to provide county planners with more than a few crop types to perform analysis on. In addition, the problem of poor/small potential vegetation location results is with the distribution of the original vegetation plantations around the island rather than the amount of records representing the vegetation type upon comparison with the climate and elevation values. If a climate change scenario was calculated for the hearts of palm crop type, few results appear on the map. For example, Figure 6-14 displays the results of the hearts of palm crop type with a precipitation increase of 10 percent and a temperature increase of one degree. However, it could also be possible that the current areas where a specific vegetation type exists may be the only the possible areas where that specific vegetation type could exist. Thus, the calculated distributions of the potential locations under current climate conditions would be as accurate as the vegetation types with a large distribution of plantations across the island under this circumstance. 


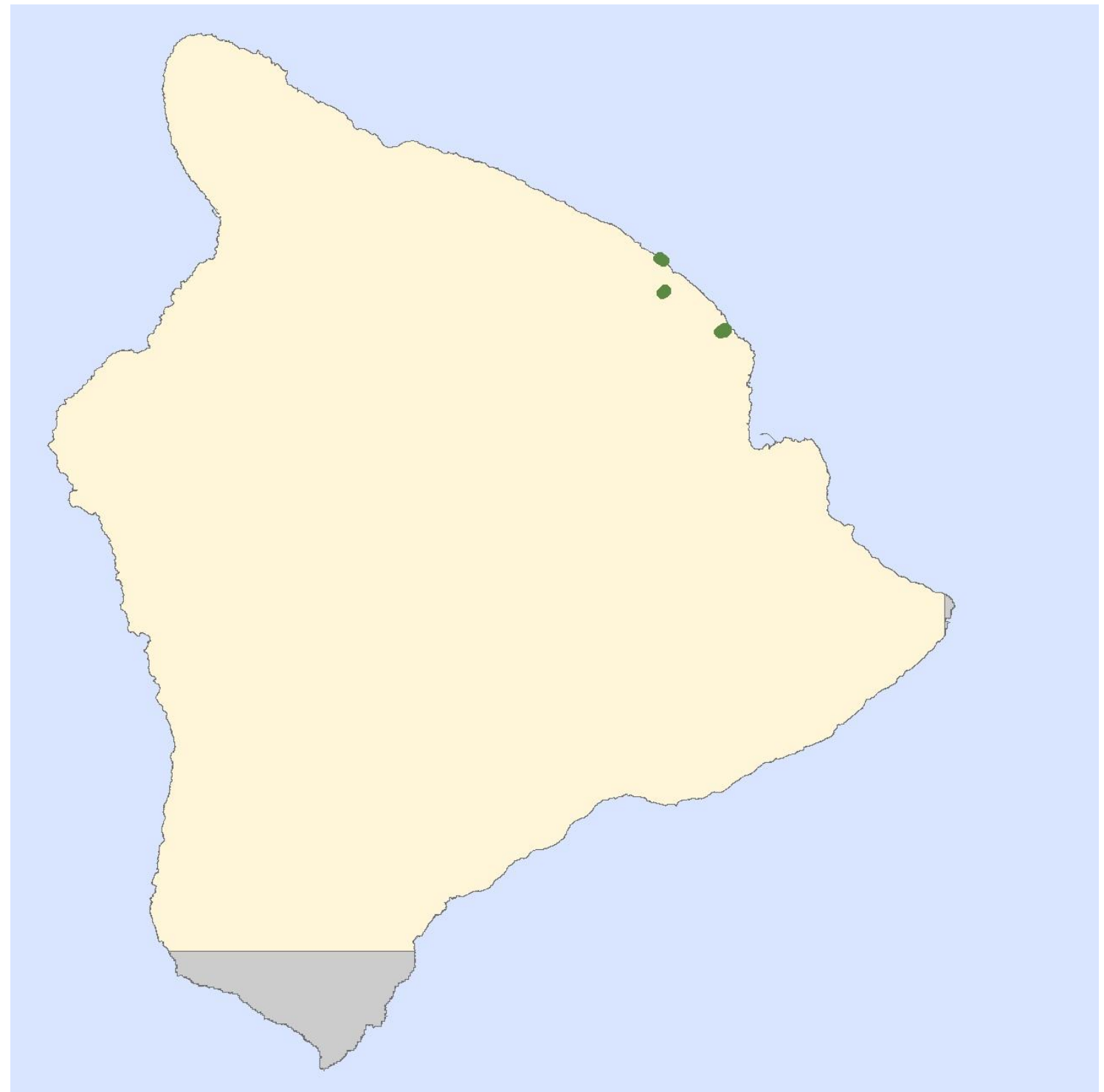

Figure 6-12: Hearts of palm plantations 


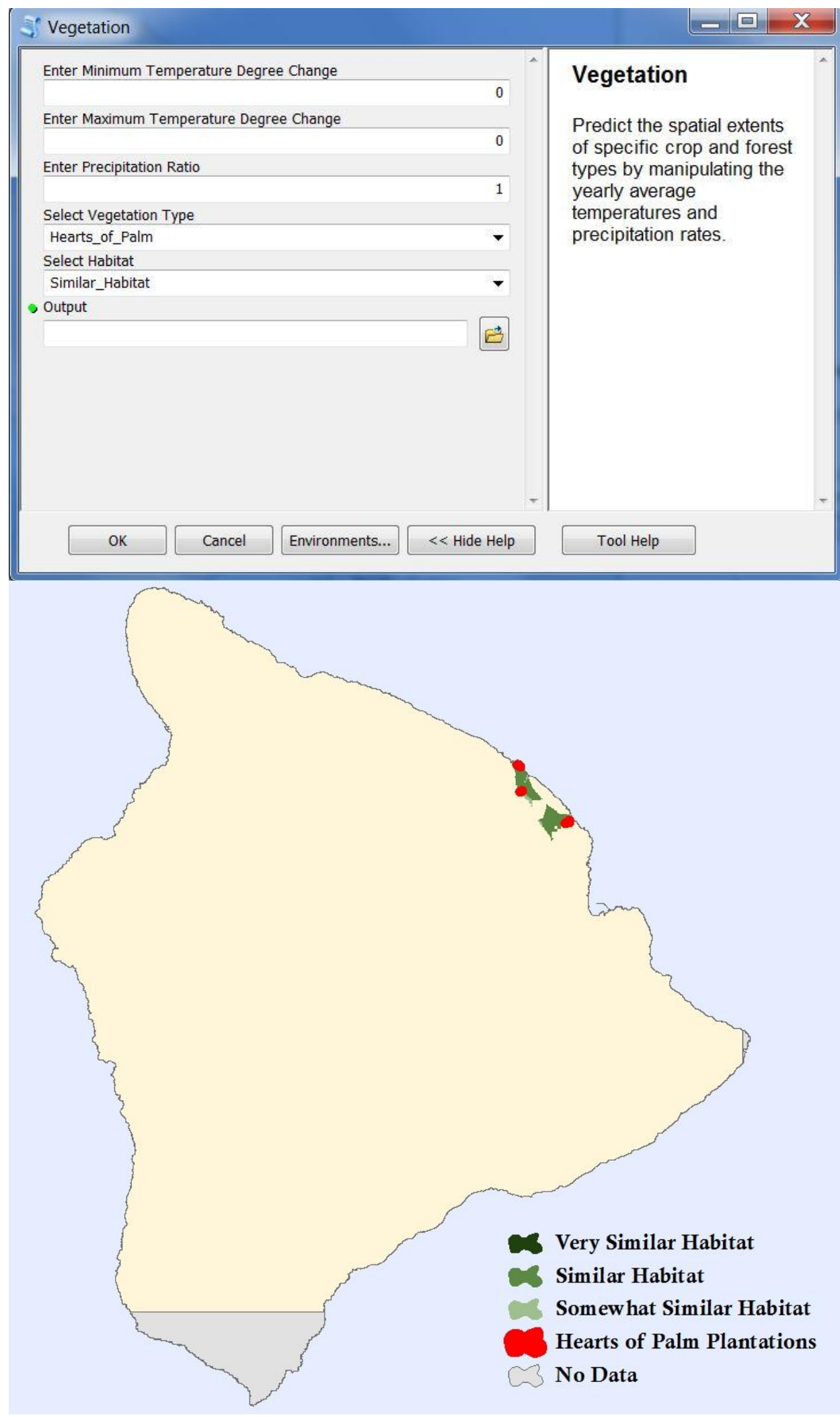

Figure 6-13: Potential hearts of palm locations under current climate conditions 

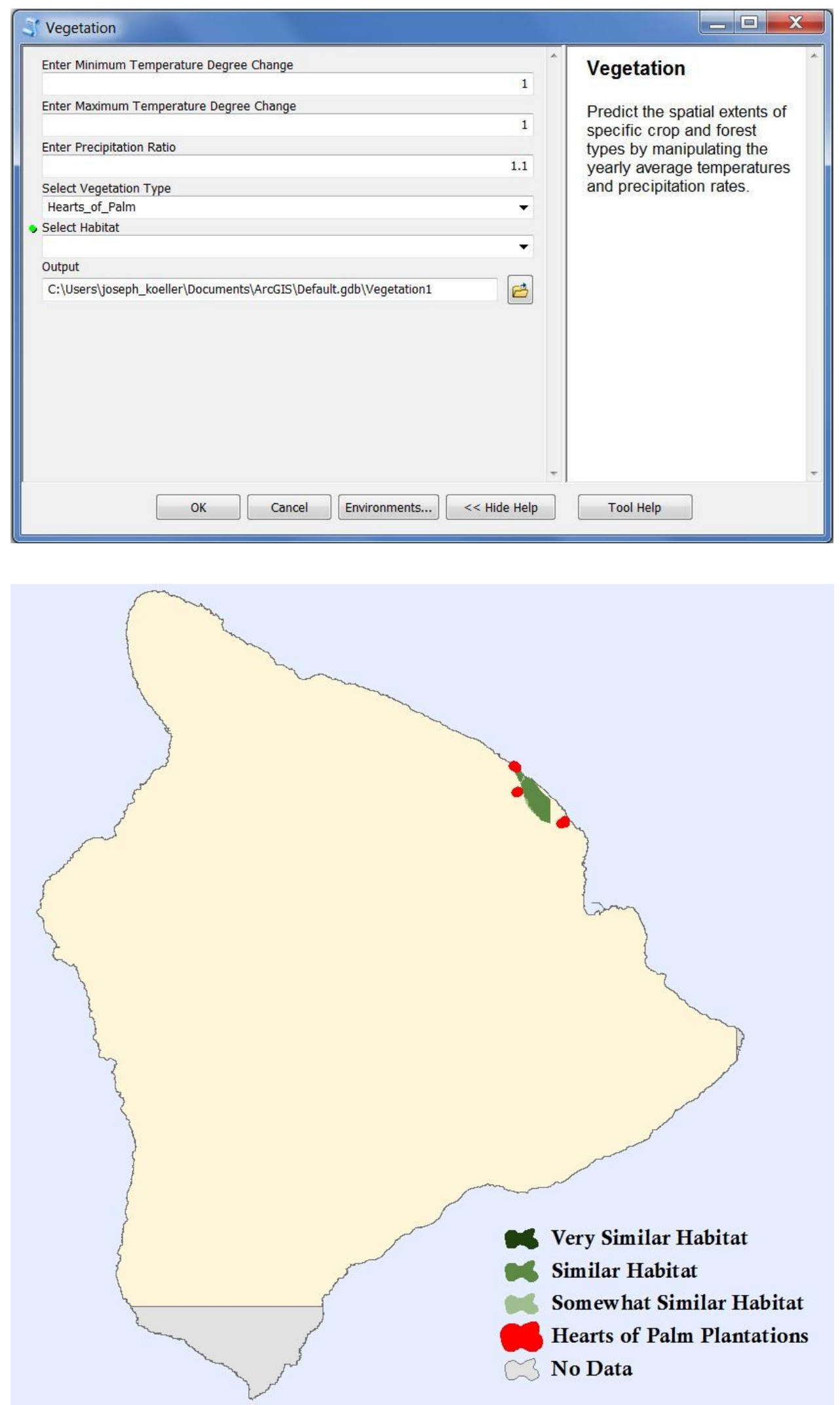

Figure 6-14: Potential hearts of palm plantations under increases in climate 
In summary, certain processes of the Hawaiian Vegetation Model went according to plan while others had to find an alternate solution. The goal of the project was to build a model that county planners could input their own climatic results into and produce possible locations of specific vegetation types. The project accomplished that goal, as the users of the project can enter a minimum and maximum temperature degree change and a precipitation ratio into the script's dialog box to produce potential locations of specific vegetation types. However, certain vegetation types in the model were underrepresented and not well dispersed when viewing the attributes of each vegetation type in the vegetation land use dataset, which resulted in a loss of accuracy when trying to predict the potential locations of those vegetation types (such as hearts of palm).

\subsection{Conclusion}

Once the Hawaiian Vegetation Model was completed, an analysis of the model was reviewed. First, the project gave a description of each input located in the scripting dialog. The scripting dialog had the inputs of minimum temperature, maximum temperature, precipitation, vegetation type, habitat type, and an output box that produced results in the model. These inputs require the user to enter a number in ratio format to predict the precipitation change and a number in degrees Fahrenheit to predict the temperature change. The script then calculates these numbers using current averages to reflect the precipitation and temperature change. The users select a vegetation and habitat type through drop down menus that represent a specific SQL statement. Once a vegetation type is selected, the user specifies a location to export the predicted vegetation areas. The county planners (or users) using the model would benefit by projecting the shifting patterns of crop and forest types when (or if) average temperature and precipitation rates change.

Once all of the user inputs were explained, examples were demonstrated in the script's dialog box. The first demonstration displayed a scenario using the specific crop type of coffee, and climate inputs of a 30 percent increase in precipitation and a two degree increase in temperatures. A comparative scenario was then demonstrated with a 10 percent decrease in precipitation, and a three degree decrease in temperatures for the coffee crops as well. The vegetation type of ohia forests was also used to test a forest type in comparison with a crop type. The ohia forests were first tested under a scenario of a 50 percent increase in precipitation, and a five-degree increase in temperatures. This scenario was compared against an opposing scenario of a 50 percent decrease in precipitation, and a five-degree decrease in temperatures. The demonstrations of the model are intended to show county planners how to run the model by example, and what sort of results to expect.

After the model was demonstrated, what went right and wrong in the project was discussed in the next section. The overall completion of the Hawaiian Vegetation Model project went as planned. This was successful because the goal was to allow the users to enter in their own climate inputs and view the altered potential locations of each individual vegetation type. What did not go according to plan was the low amount of representative samples for some of the vegetation types, as well as the lack of distance between each sample, which caused a loss of accuracy when predicting the potential locations. 



\section{Chapter 7 - Conclusions and Future Work}

\subsection{Introduction and Project Goals Review}

The completion of the Hawaiian Vegetation Model satisfied the project's primary goal of predicting new vegetation patterns from climate changes. The project reached the goal by finding the necessary datasets of temperature averages, precipitation averages, vegetation types, and elevation, then, performing the necessary comparison analysis to extract climate and elevation data ranges for the vegetation types. Mathematical equations were set up to allow users to incorporate different temperature and precipitation averages.

Predictive areas of specific vegetation types resulted from the user's own temperature and precipitation inputs. However, the potential locations of the vegetation types experienced areas of accuracy loss due to the lack of spatial distribution of some vegetation types.

The accomplishment of the primary goal allowed the intended audience of county planners to save time from having to perform their own vegetative analysis. This goal was created because with increasing carbon dioxide levels in the atmosphere, climate change issues are a growing concern on the planet. The primary resources of food (vegetation related) for humans, will most likely shift locations, or alter in quantities due to these climate changes. The above goal intended to give an idea of what to expect from the vegetation if the climate does change in the future.

\subsection{Goal Accomplishment}

Accomplishing the goal first required researching which variables had the greatest impact on the Hawaiian vegetation. In the article "A Methodological Approach of Climatological Modeling of Air Temperature and Precipitation through GIS Techniques," Ninyerola, Pons, and Roure used current precipitation and temperature averages to determine the future climates in Northeast Spain. Based on the article, the Hawaiian Vegetation Model project also used these two variables. Elevation also had in important impact on vegetation, as referenced by the client of this project. To predict where the specific vegetation areas would grow, this project needed a dataset with individual vegetation types. The Redlands Institute provided a remotely sensed dataset showing the locations of specific crop and forest types. This dataset was compared to the other datasets of temperature, precipitation, and elevation by overlaying the vegetation dataset onto the aggregated climate and elevation dataset. The overlays created specific ranges of temperature, precipitation, and elevation, which were used to discover each vegetation types' percentile range, establishing the ranges. Once the ranges were established, any predicted change in temperature and precipitation averages could change the potential locations of each vegetation type by using an SQL query to select the new areas.

\subsection{Project Results}

Upon completion of the comparative results between the vegetation, climate, and elevation datasets, eight crop types and five forest types were used in this project. The 13 vegetation types (crop and forest types combined) allowed the user to manipulate average 
temperature and precipitation rates to view the changes to the potential locations of each of these vegetation types. Accuracy loss for determining the positions of each vegetation type could have resulted because more complex formulas could calculate uneven changes in precipitation rates over a surface, as opposed to uniform distributions of changes, which this project used. In addition, when performing the comparative analysis, some of the crop types with compact actual distributions on Hawaii had very small temperature, precipitation, and elevation data ranges. This also resulted in accuracy loss since it is very difficult to predict the future location of a vegetation type with very limited climate and elevation data ranges.

\subsection{Future Work}

Once the Hawaiian Vegetation Model was completed, three project suggestions arose as continuations to this project. The first suggestion was to create a web interface from the model and script already created. This would allow the project to reach a wider audience, including county planners who do not have a license to run ArcGIS software, or do not want to deal with downloading the ArcGIS Explorer and Python version of this project. The client originally suggested building a web interface as a means to communicate the results of the models. However, due to time and error constraints of the geoprocessing tool on the web interface, it was only possible to place samples of the models online.

A second possible extension is to create a new remote sensing analysis to obtain a more accurate/new vegetation dataset. Again, the client also suggested this, but time constraints hampered the development of a new dataset. These refinements would not have to take place in the same study area. The flexibility on this project can include any study area, as long as appropriate data sources are available.

A third possible area of future work would be to rework the definition of very similar, similar, and somewhat similar by considering the areas of the polygons rather than the numbers, as it was described in section 5.3.1. All of these points were calculated solely on the numbers of polygons, not their areas. A more precise approach would consider the areas of these polygons, for example, identify the polygons near the median that accounted for the middle 40 percent by area. 


\section{Works Cited}

Austin, A. (2002). Differential effects of precipitation on production and decomposition along a rainfall gradient in Hawaii. Ecology, 83(2), 328-338.

Bell, Martin, \& Walker, Mike. (2005). Late Quaternary Environmental Change: Physical and Human Perspectives. Upper Saddle River: Pearson/Prentice Hall.

Giorgi, F., Brodeur, C., \& Bates, G. (1994). Regional climate change scenarios over the United States produced with a nested regional climate model. Journal of Climate, 7 , 375-399.

Goodchild, M. (1992). Geographical data modeling. Computer \& Geosciences, 18(4), 401408.

Kravchenko, A., \& Bullock, D. (1999). A comparative study of interpolation methods for mapping soil properties. Agronomy Journal, 91, 393-400.

Ninyerola, M., Pons, X., \& Roure, J. (2000). A methodological approach of climatological modeling of air temperature and precipitation through GIS techniques. International Journal of Climatology, 20, 1823-1841.

Rogerson, P. (2006). Statistical Methods for Geography: A Student's Guide. London: SAGE Publications Ltd.

Soille, P., Vogt, J., \& Colombo, R. (2003). Carving and adaptive drainage enforcement of grid digital elevation models. Water Resources Research 39(12), 1-13.

Soller, D., Berg, T., \& Stamm, N. (2004). National geologic map database project: overview and progress. Digital Mapping Techniques '04, 1451, 1.

Tsichritzis, D., \& Lochovsky, F. (1977). Database Management Systems. New York: Academic Press. 



\section{Appendices}

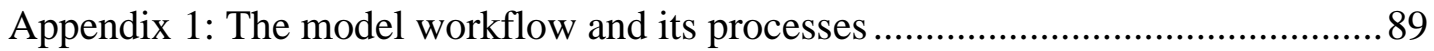

Appendix 2: Locations of collected temperature data .......................................... 90

Appendix 3: Locations of collected precipitation data ....................................... 91

Appendix 4: The script used to run the processes of the model in ArcGIS ...............92

Appendix 5: The script used to run the processes of the model in ArcGIS Explorer 93

Appendix 6: The script dialog box and its instruction ............................................ 94

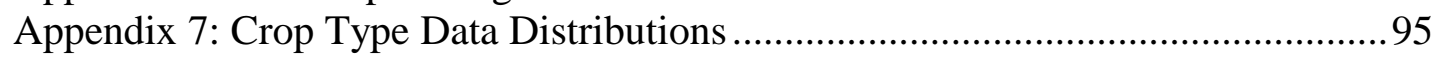

Figure A-7-1: Avocado Data Distributions.................................................... 95

Figure A-7-2: Banana Data Distributions ................................................. 96

Figure A-7-3: Coffee Data Distributions ....................................................97

Figure A-7-4: Hearts of Palm Data Distributions ........................................... 98

Figure A-7-5: Macadamia Nut Data Distributions ...........................................99

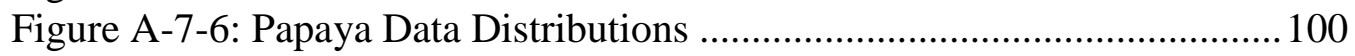

Figure A-7-7: Sweet Potato Data Distributions .......................................... 101

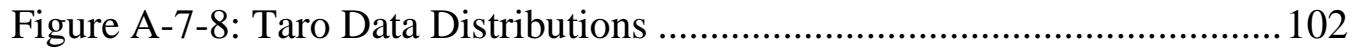

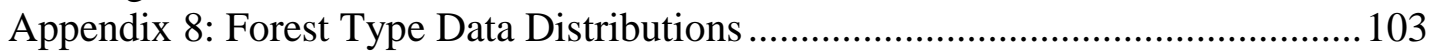

Figure A-8-1: Alien Tree and Plantation Forest Data Distributions................. 103

Figure A-8-2: Eucalyptus Forest Data Distributions ..................................... 104

Figure A-8-3: Koa Forest Data Distributions ............................................... 105

Figure A-8-4: Mamane Forest Data Distributions ....................................... 106

Figure A-8-5: Ohia Forest Data Distributions ............................................ 107

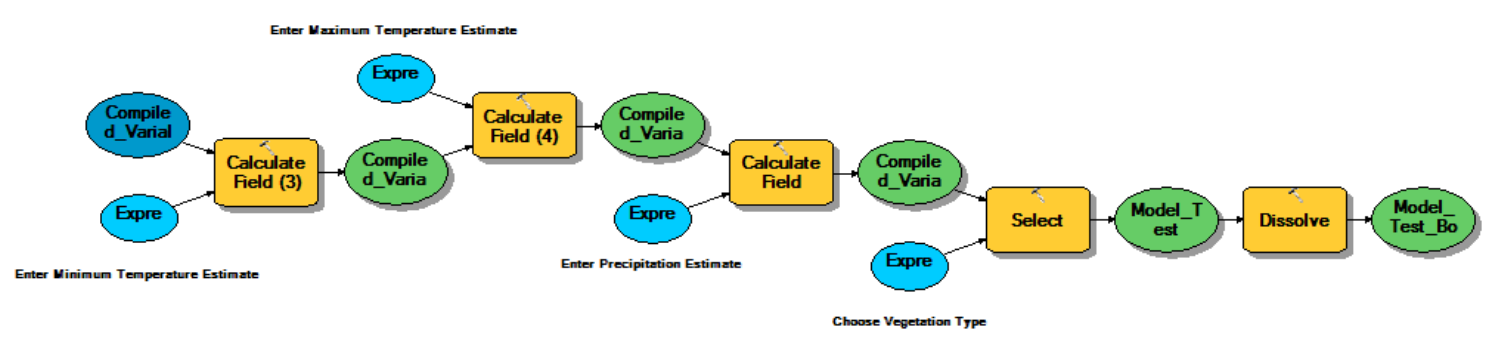

Appendix 1: The model workflow and its processes 


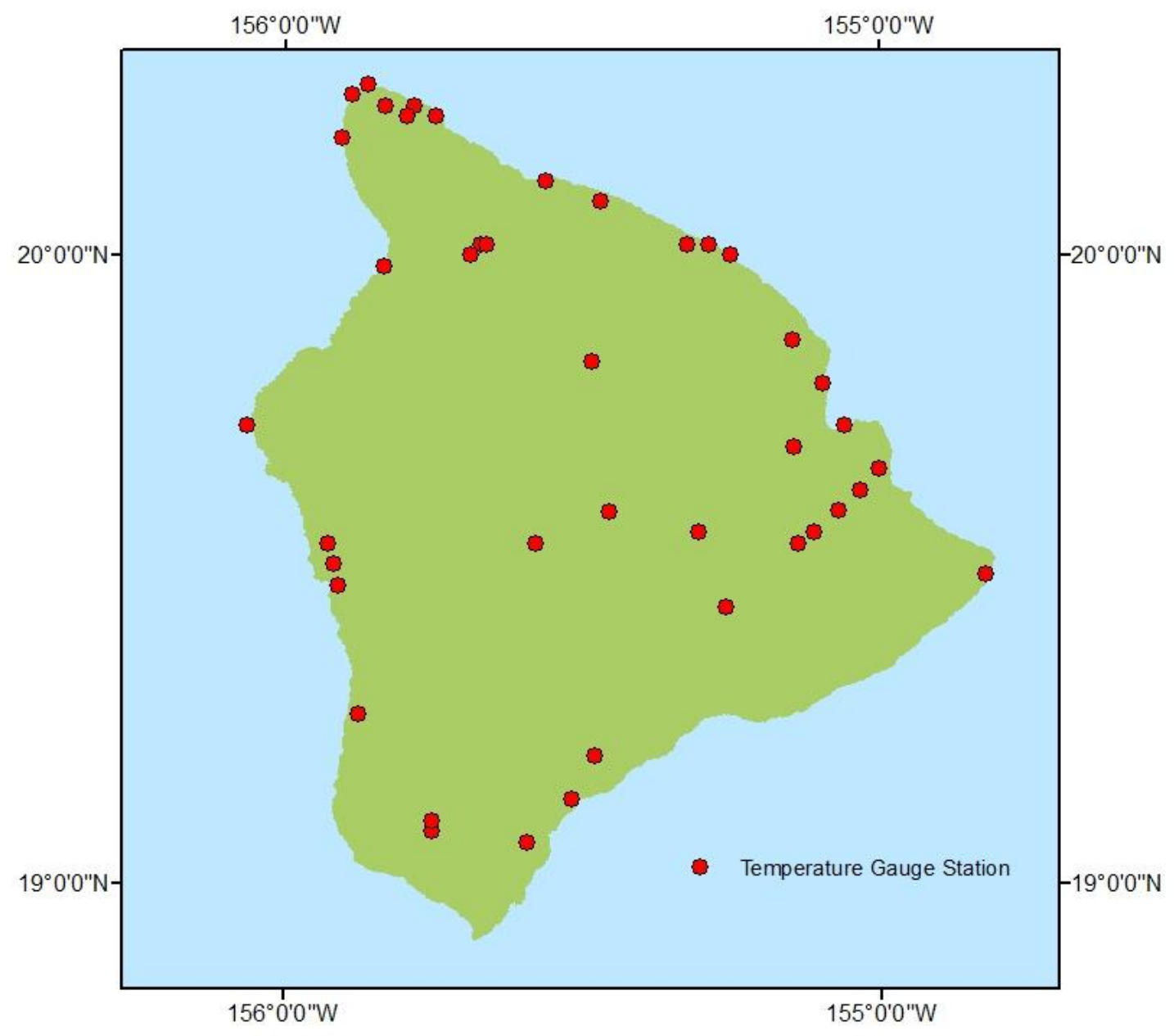

Appendix 2: Locations of collected temperature data 


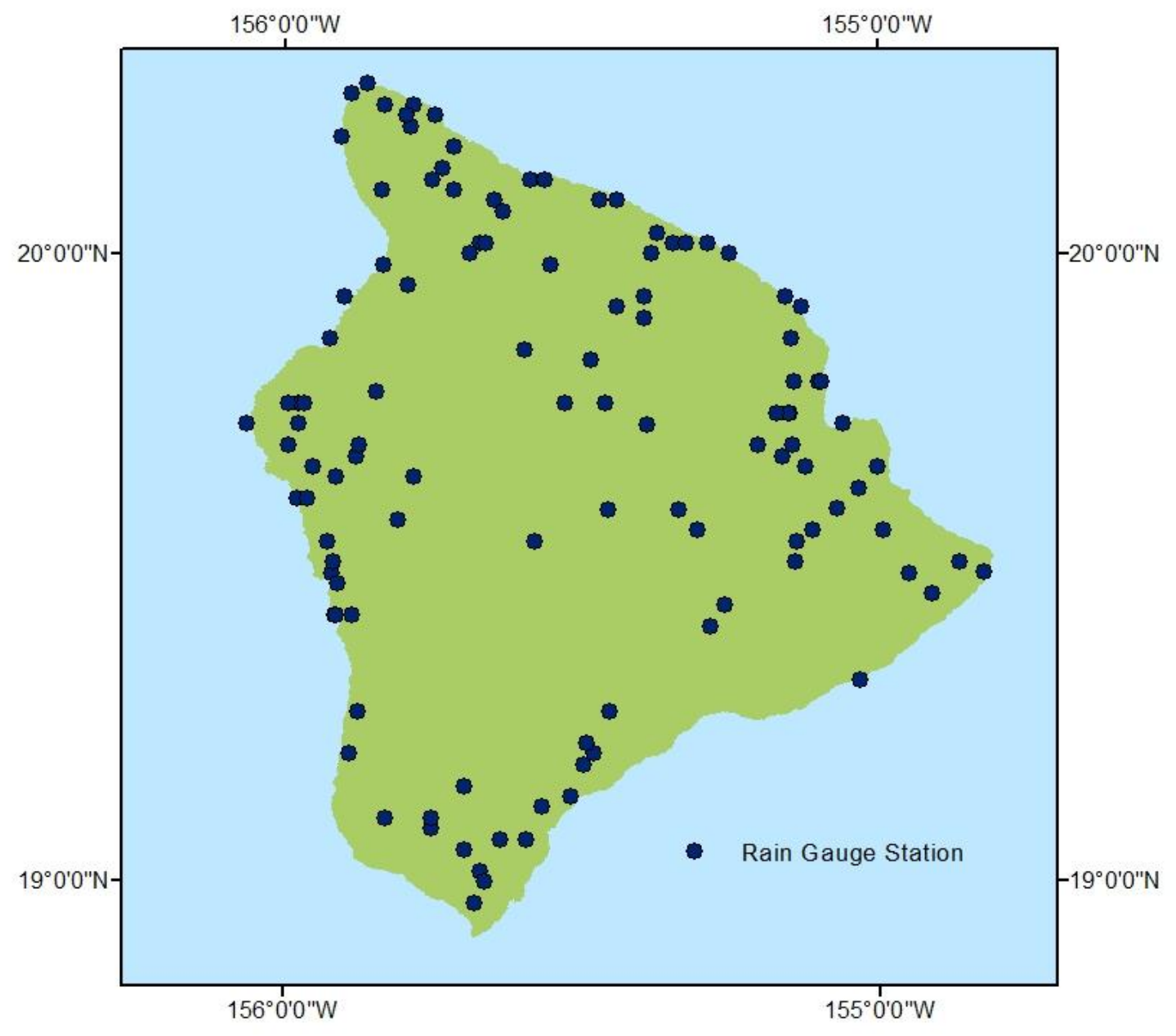

Appendix 3: Locations of collected precipitation data 


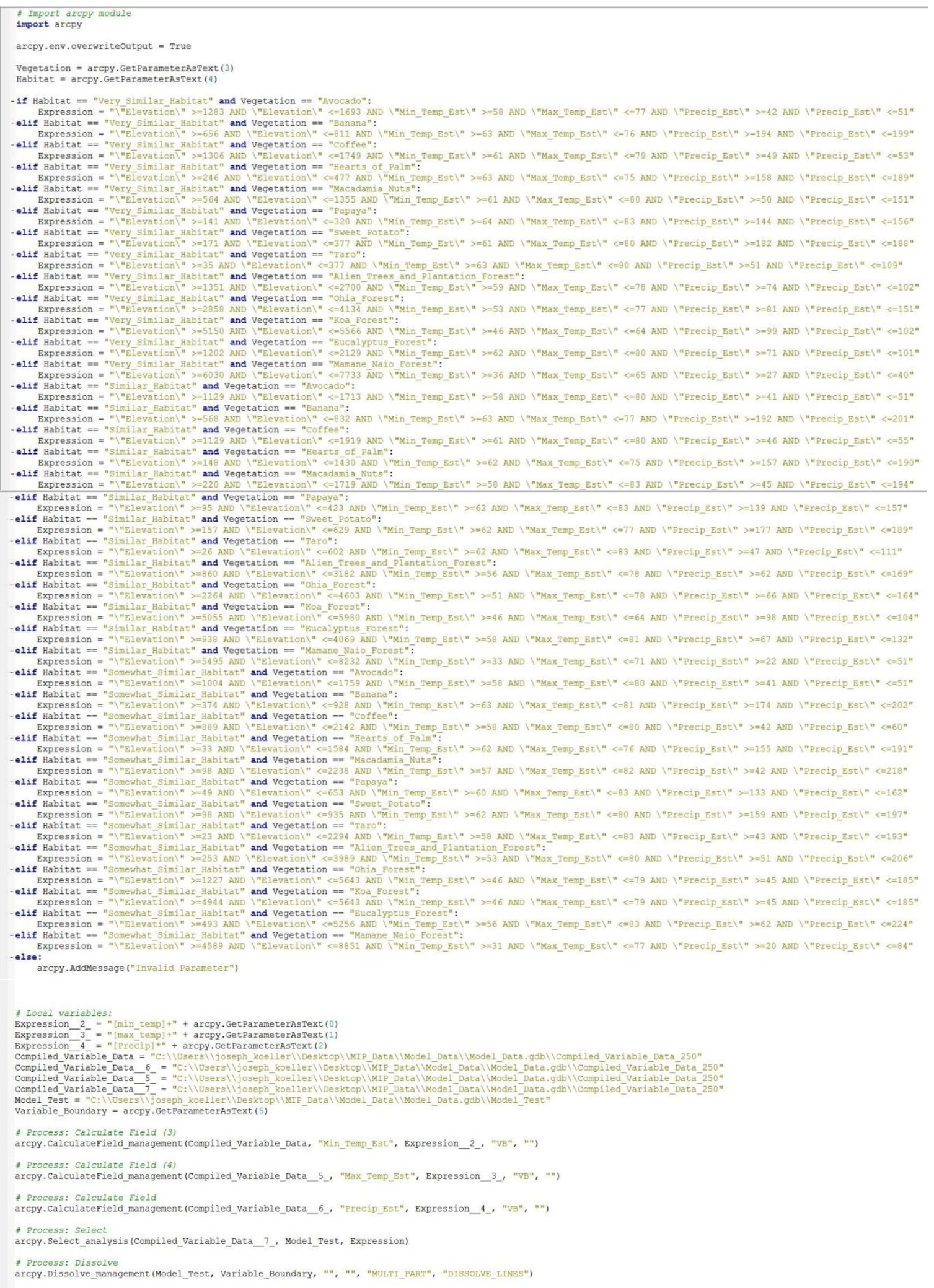

Appendix 4: The script used to run the processes of the model in ArcGIS 


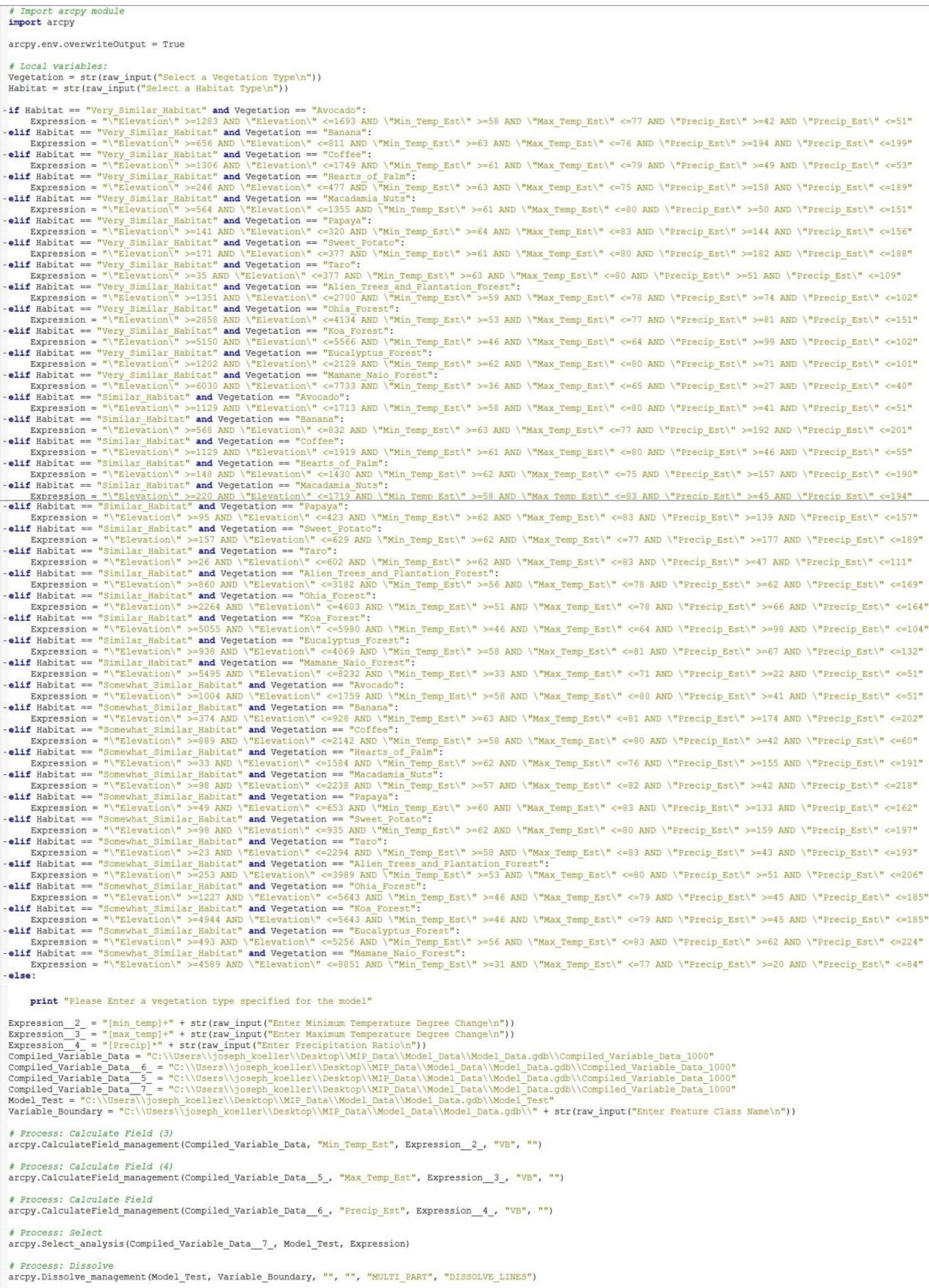

Appendix 5: The script used to run the processes of the model in ArcGIS Explorer 


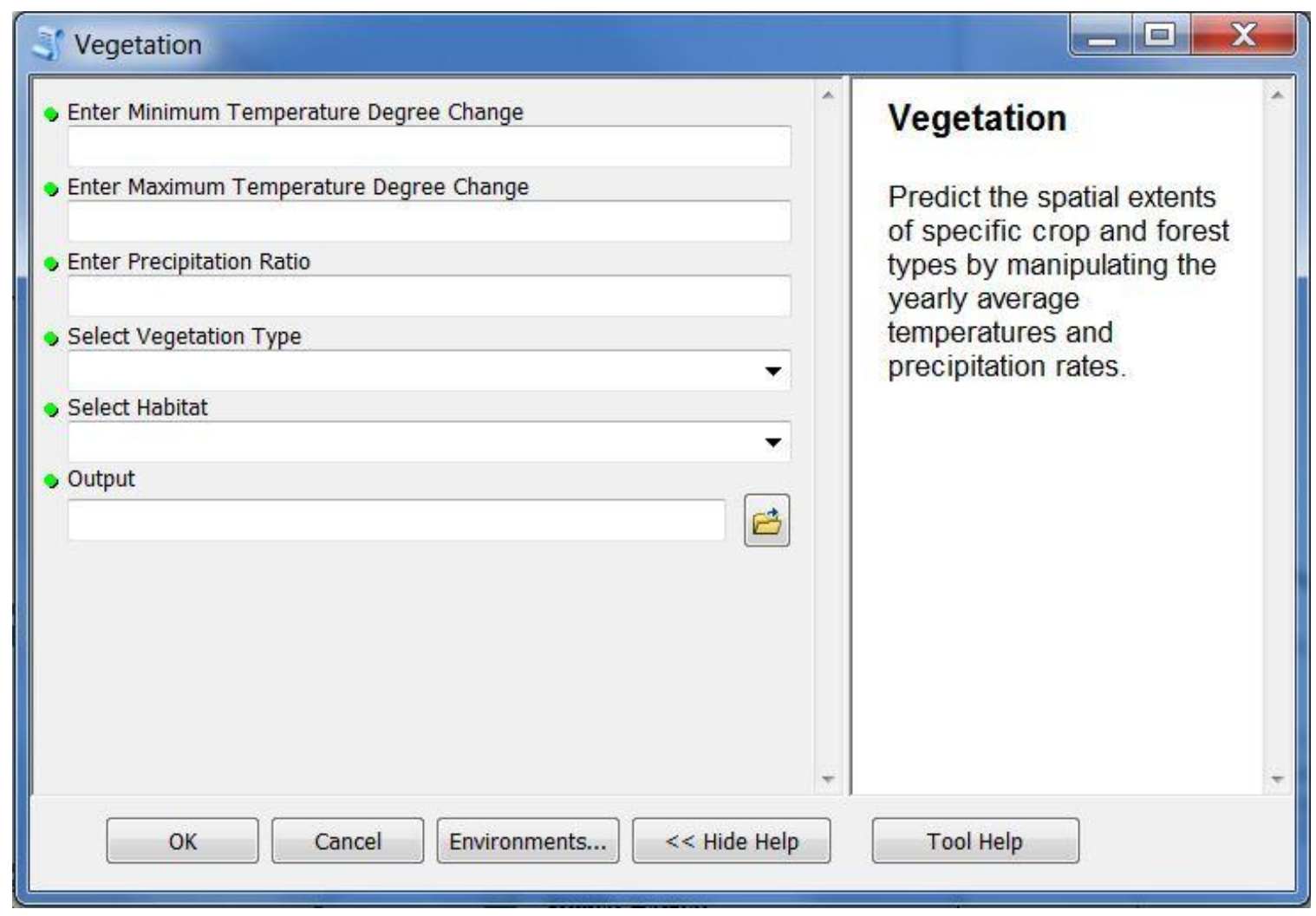

Appendix 6: The script dialog box and its instruction 


\section{Appendix 7: Crop Type Data Distributions}
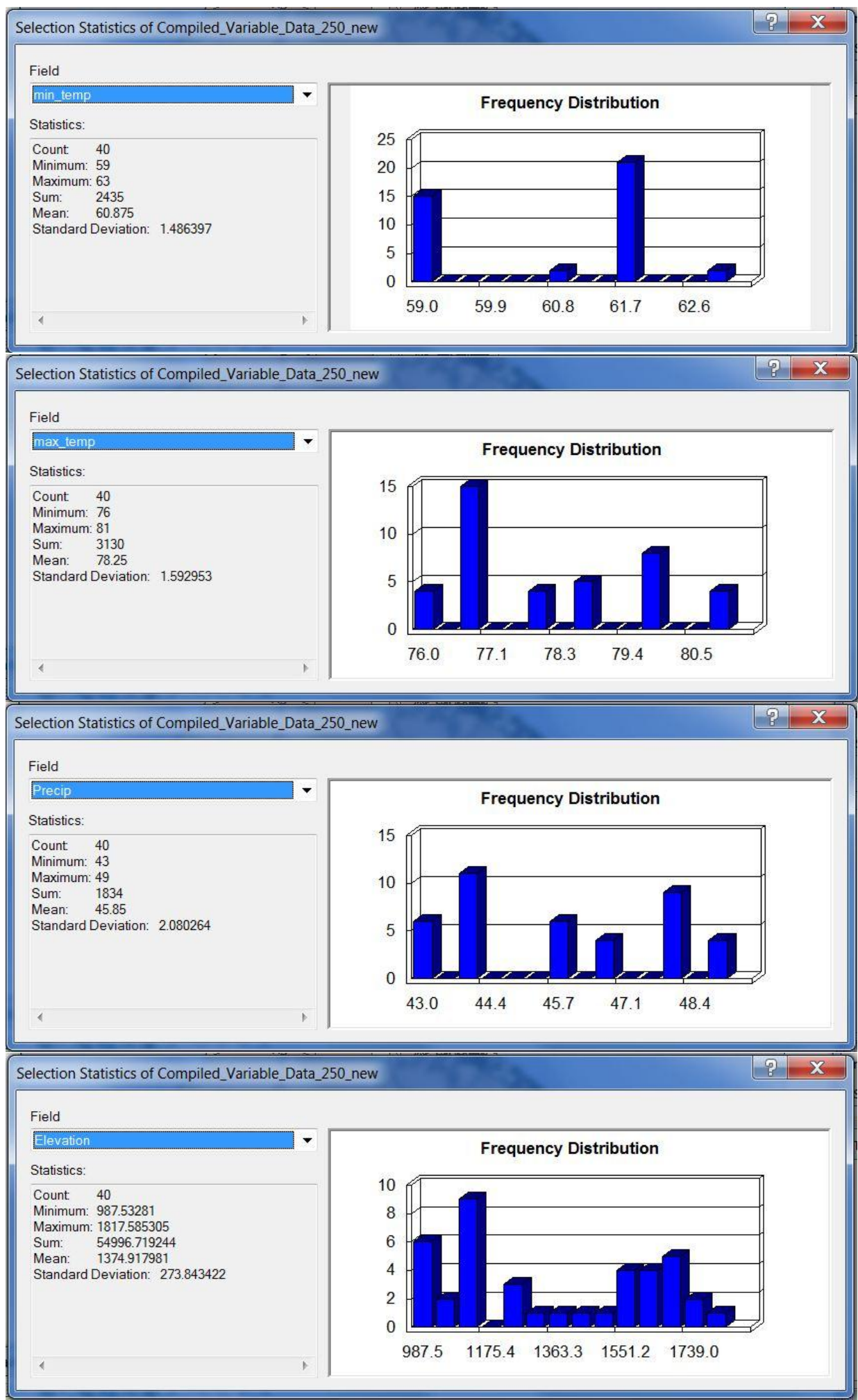

Figure A-7-1: Avocado Data Distributions 


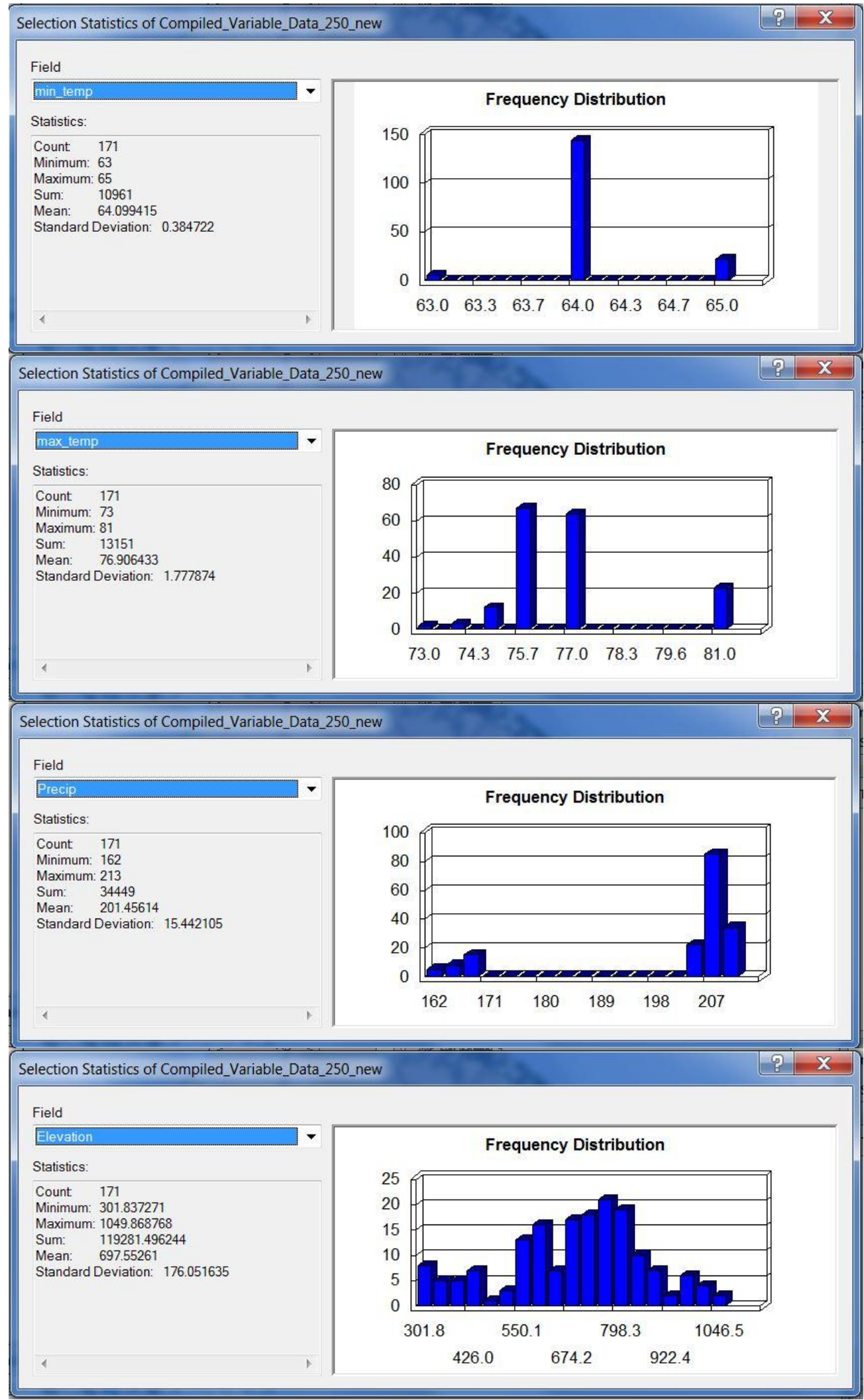

Figure A-7-2: Banana Data Distributions 

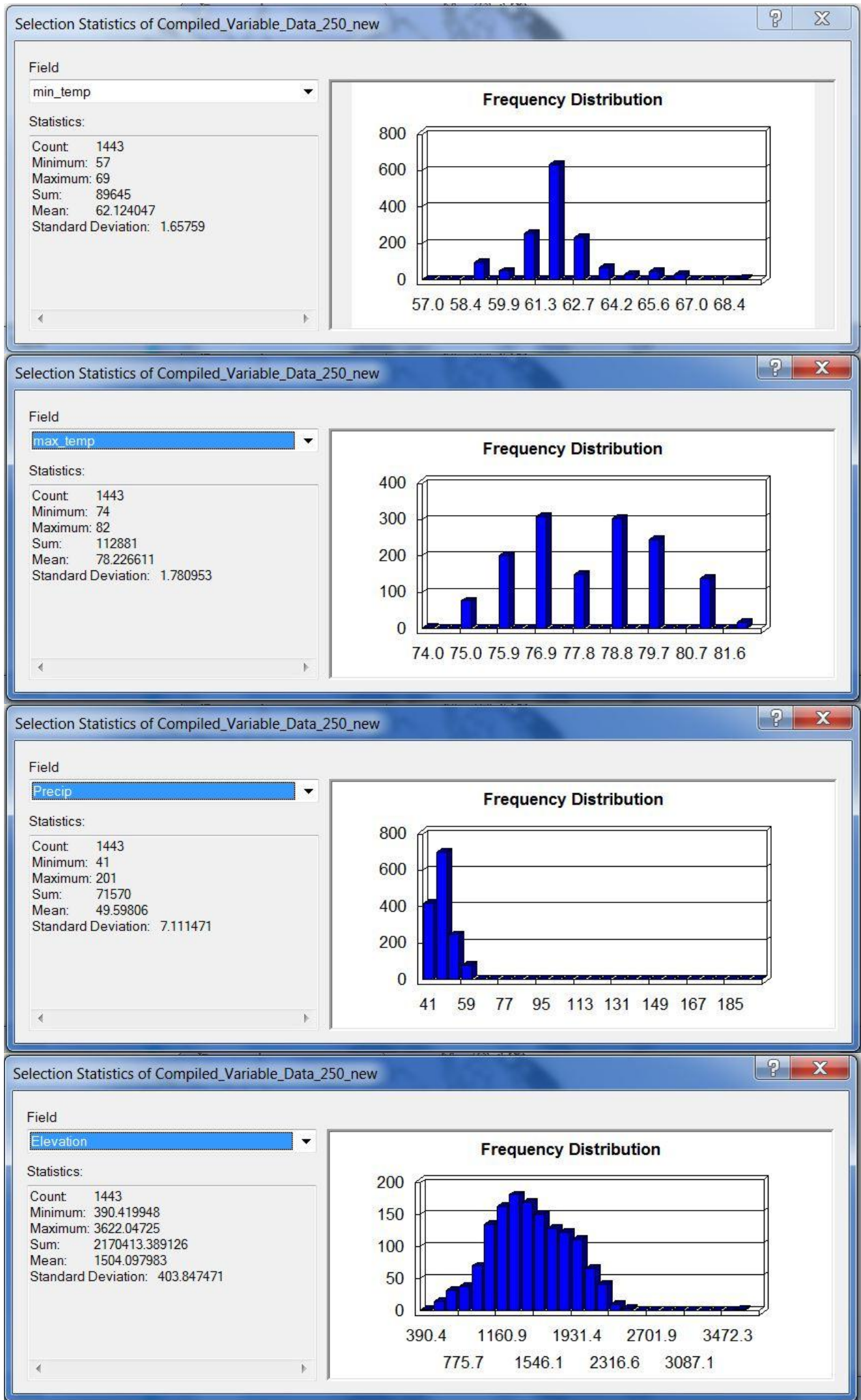

Figure A-7-3: Coffee Data Distributions 


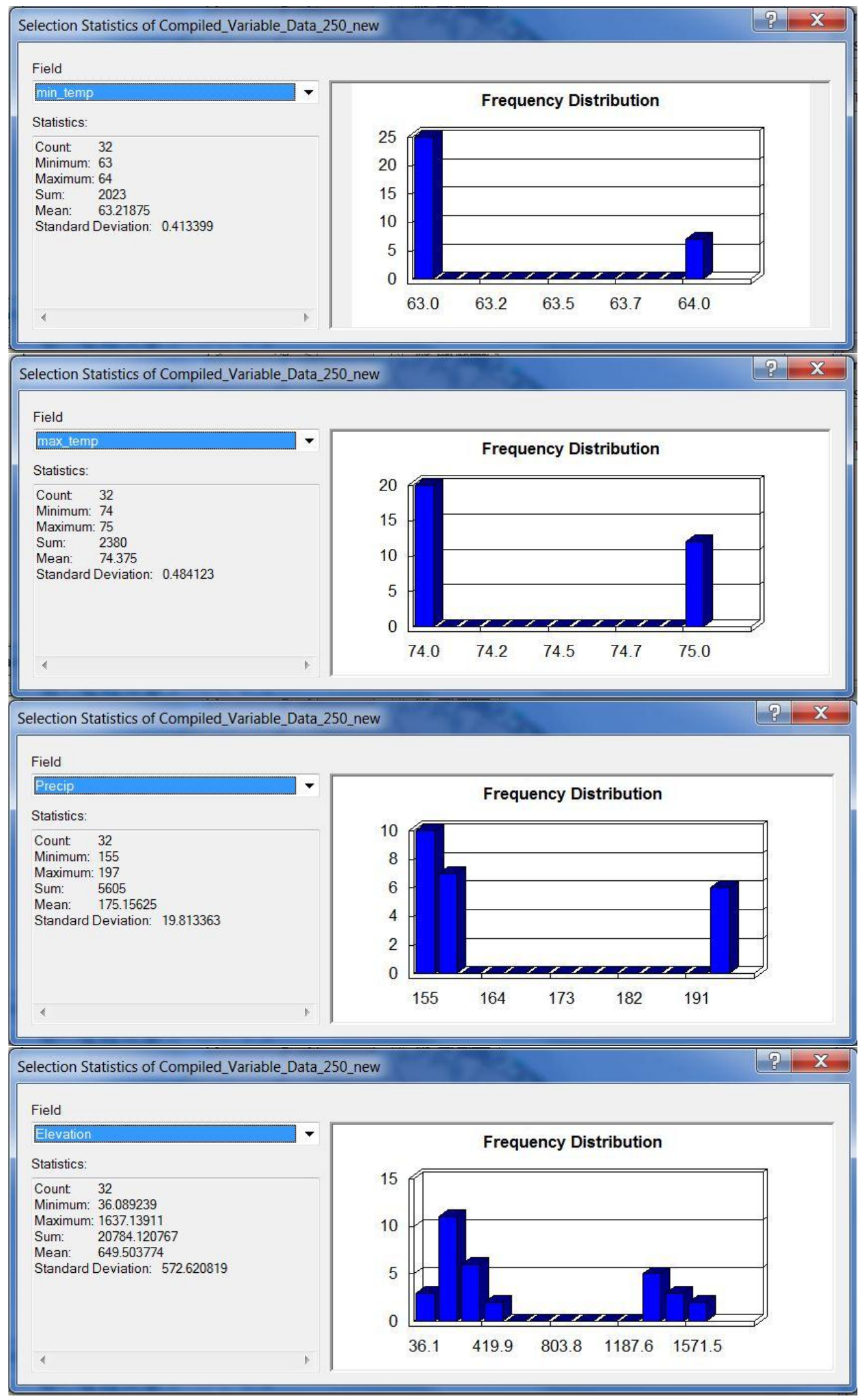

Figure A-7-4: Hearts of Palm Data Distributions 


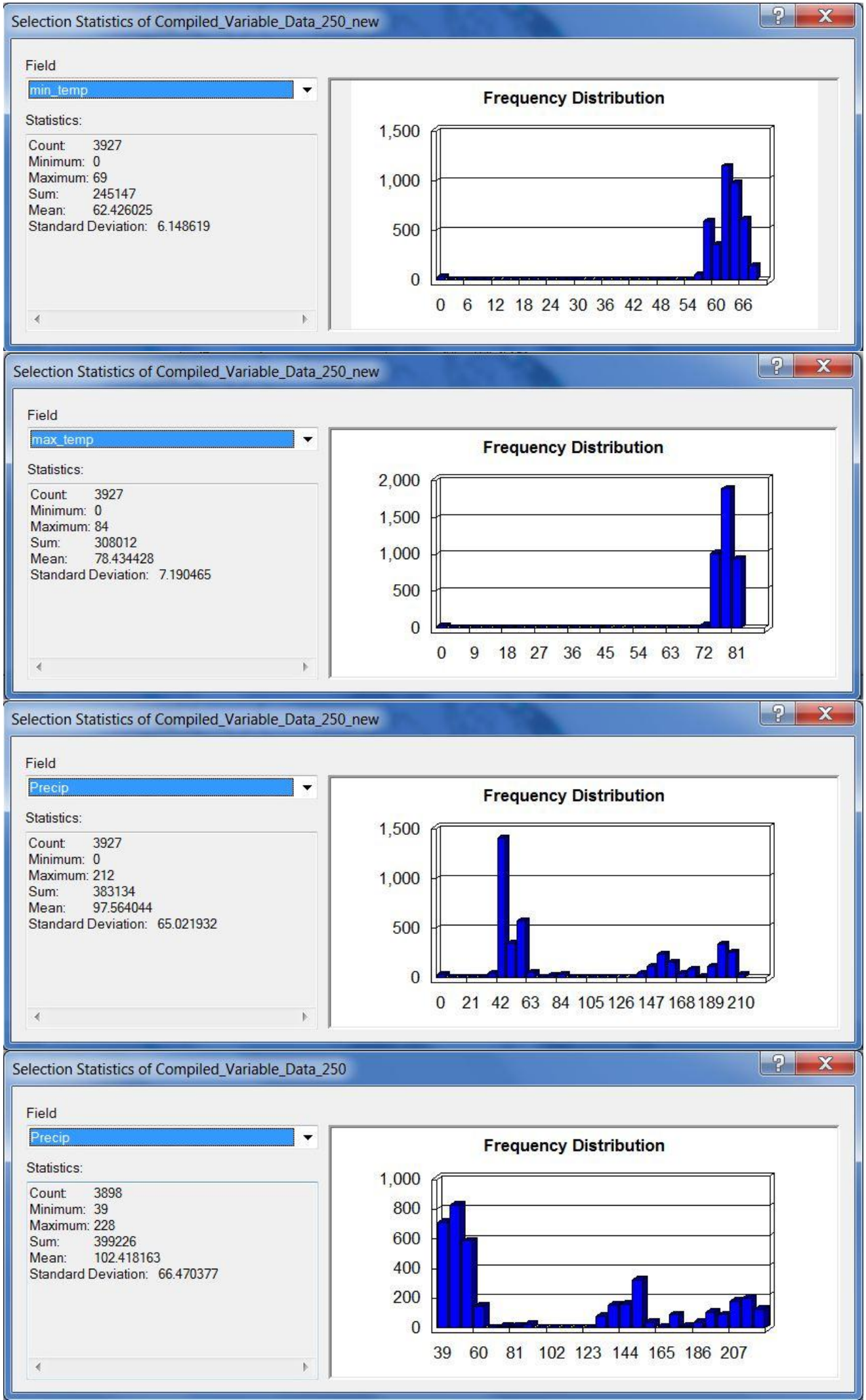

Figure A-7-5: Macadamia Nut Data Distributions 


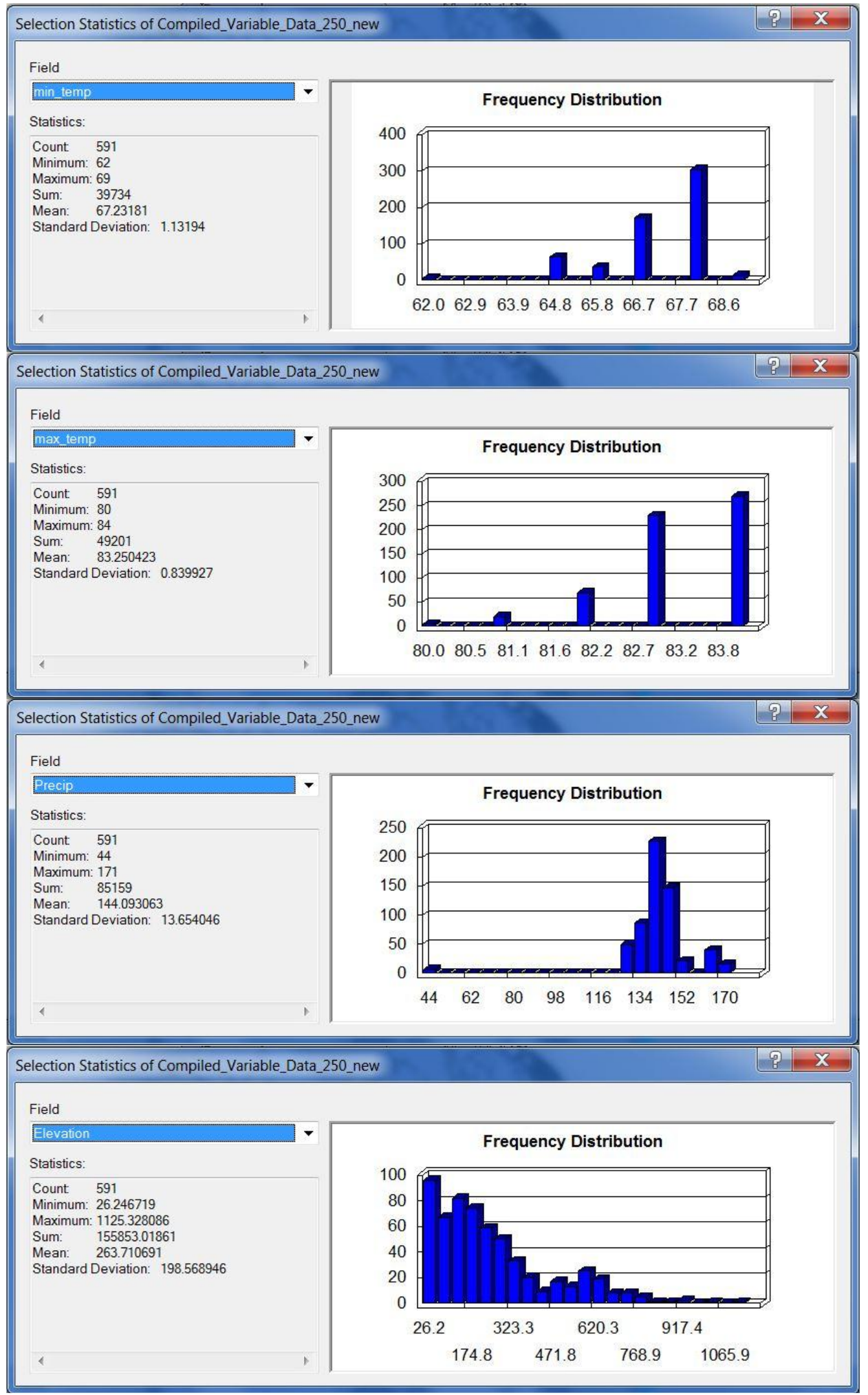

Figure A-7-6: Papaya Data Distributions 

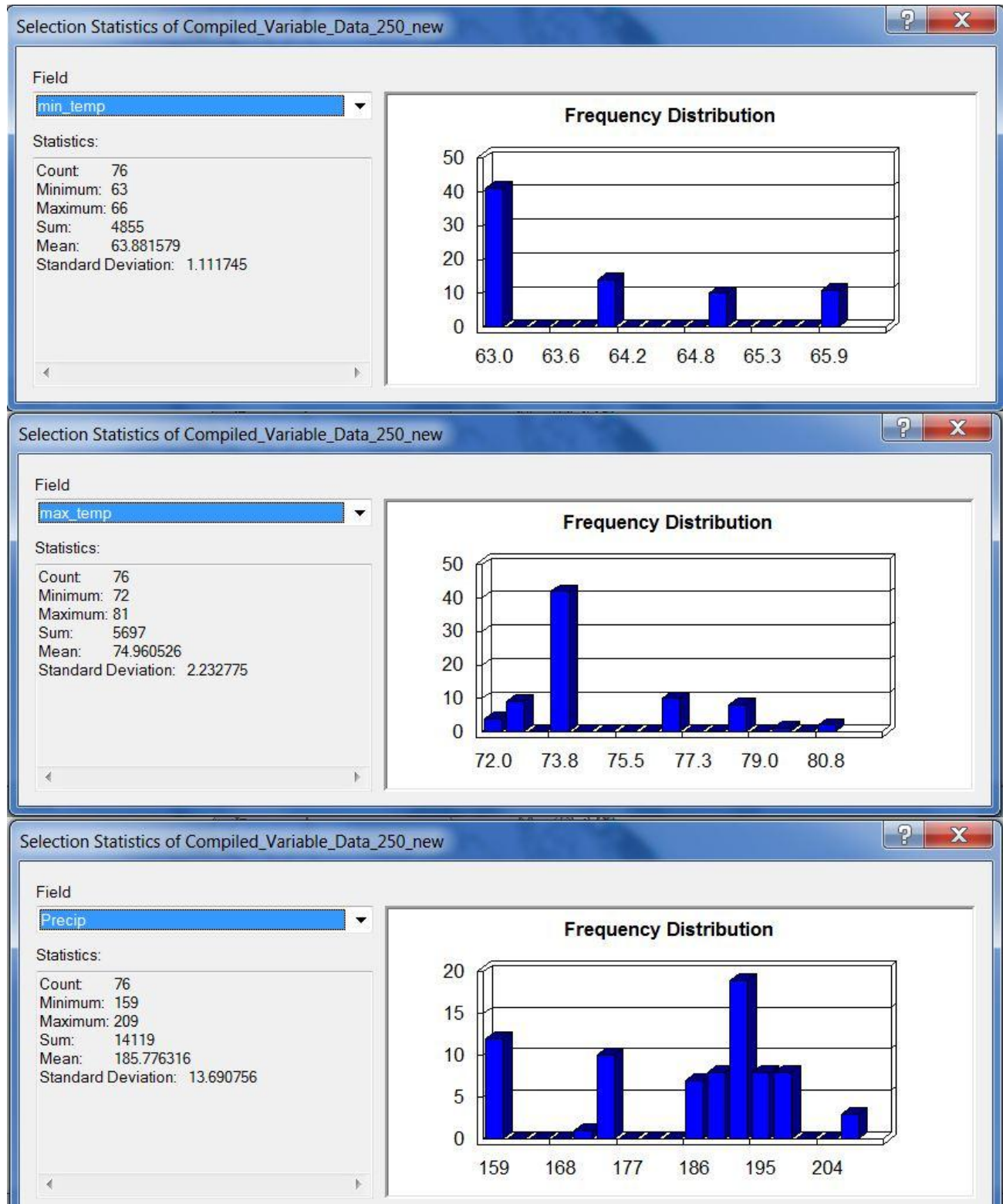

Selection Statistics of Compiled_Variable_Data_250_new

(? $\mathrm{X}$

$$
\text { Field }
$$

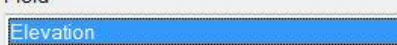

Statistics:

Count 76

Minimum: 59.055118

Maximum: 1056.430448

Sum: $\quad 27198.162771$

Mean: $\quad 357.870563$

Standard Deviation: 272.181092

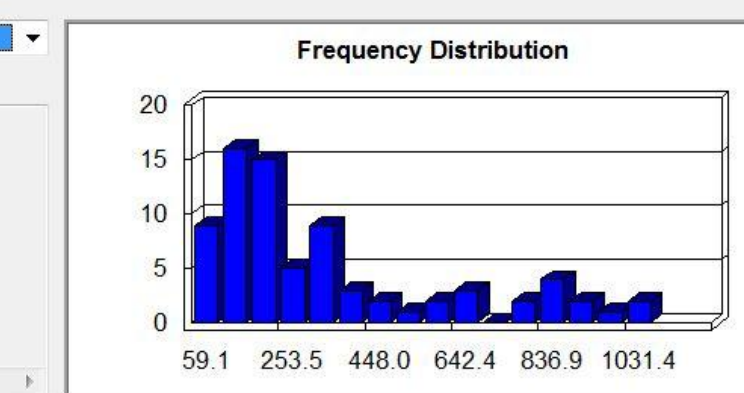

\section{Figure A-7-7: Sweet Potato Data Distributions}




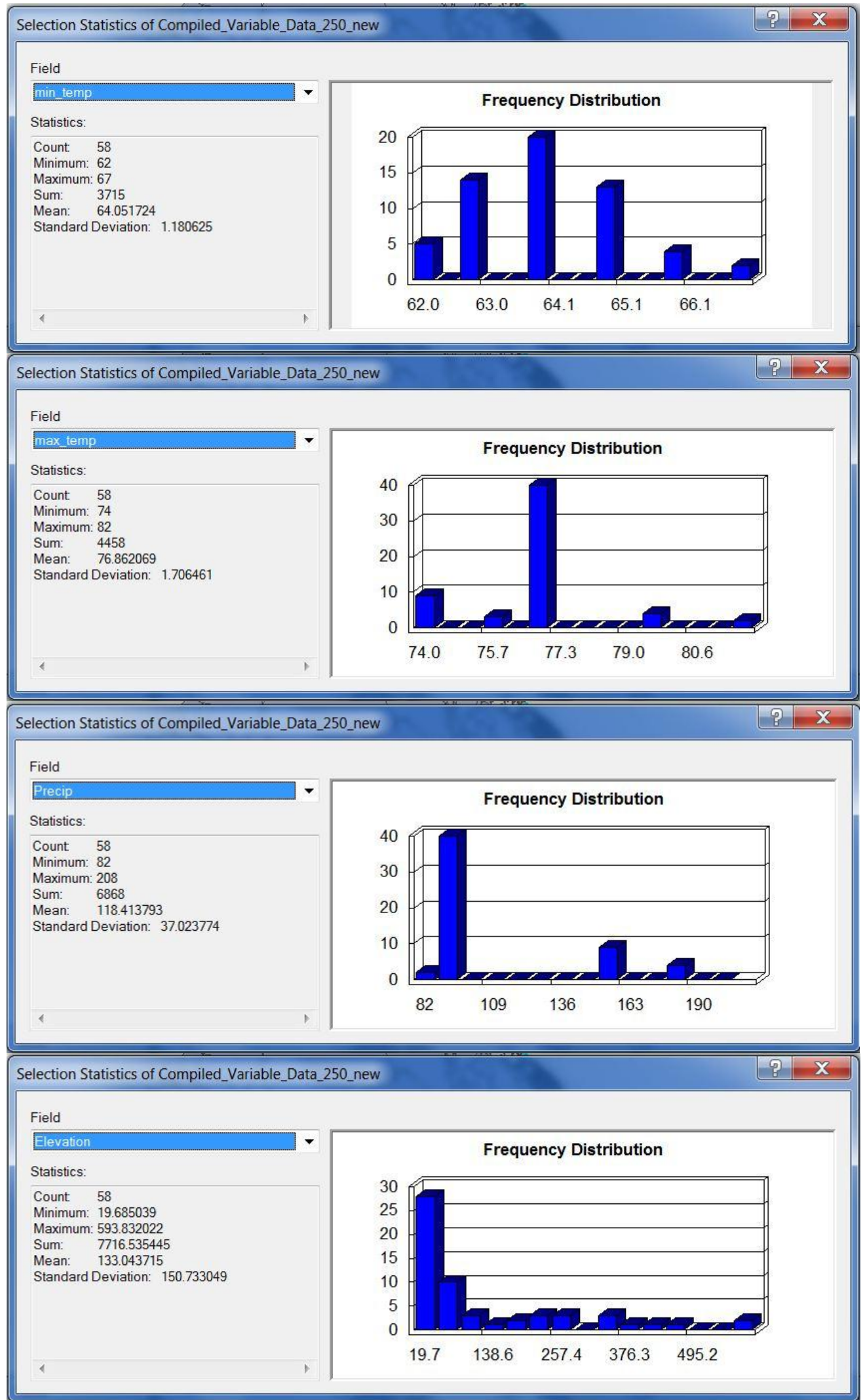

Figure A-7-8: Taro Data Distributions 


\section{Appendix 8: Forest Type Data Distributions}
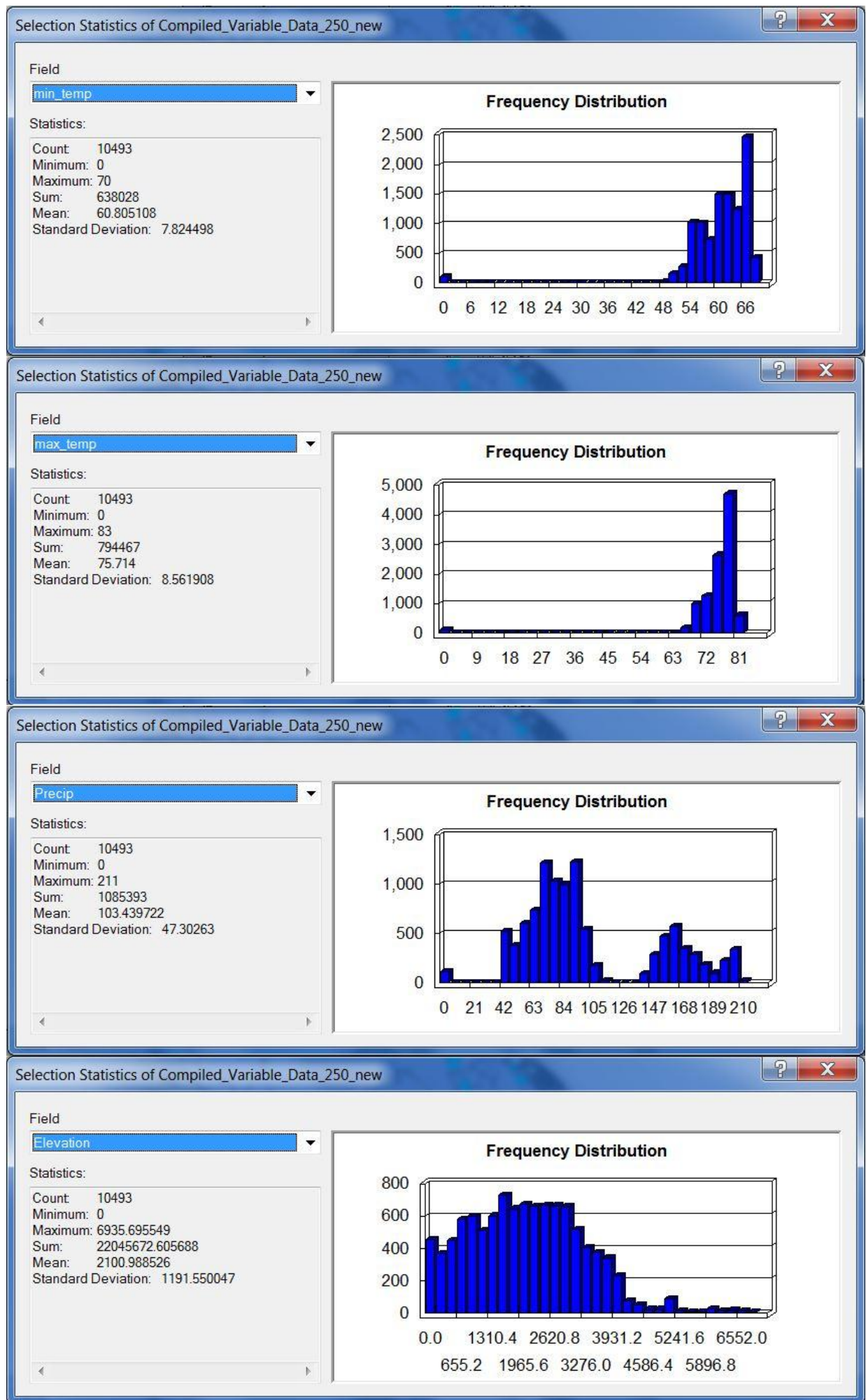

Figure A-8-1: Alien Tree and Plantation Forest Data Distributions 


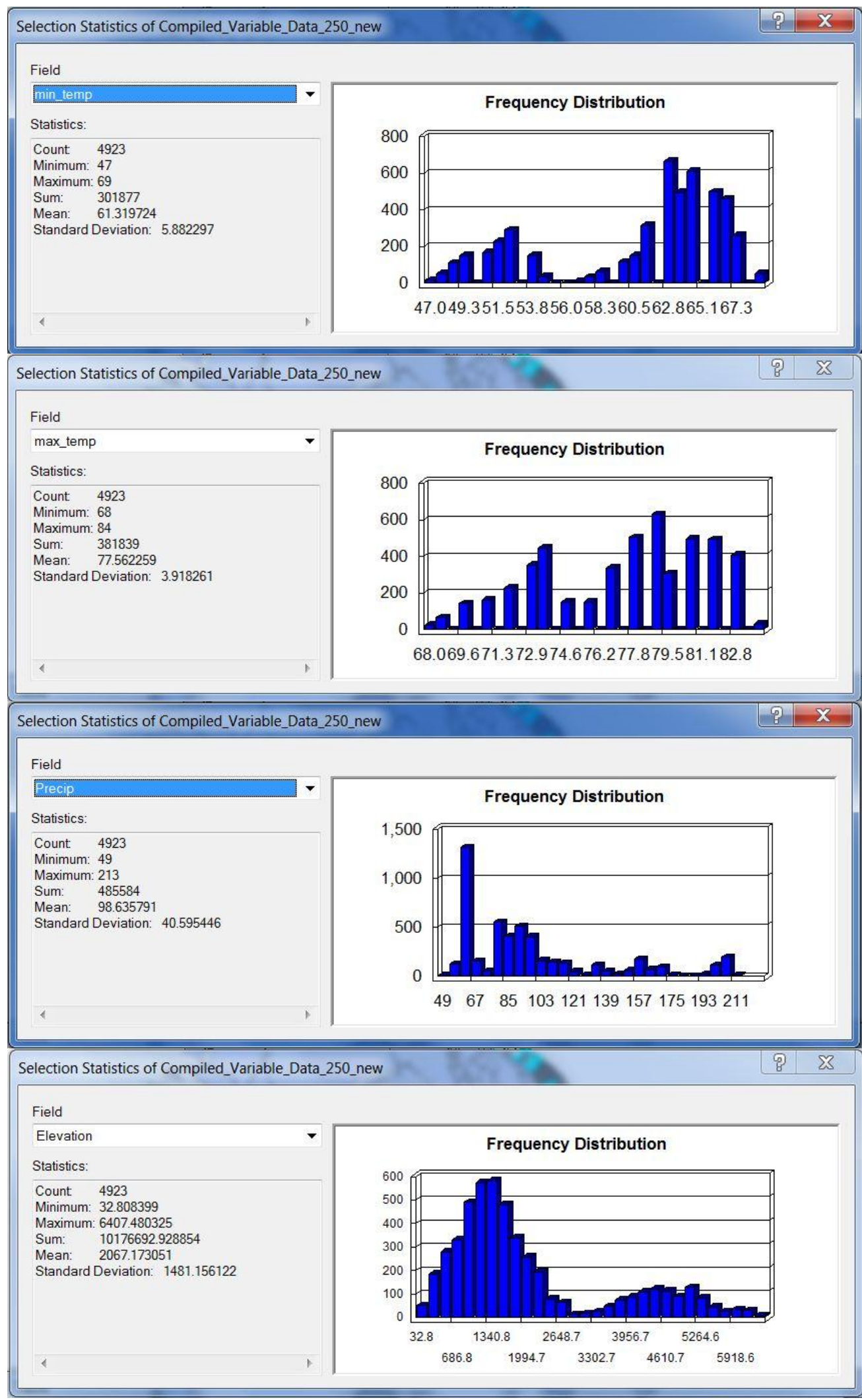

Figure A-8-2: Eucalyptus Forest Data Distributions 


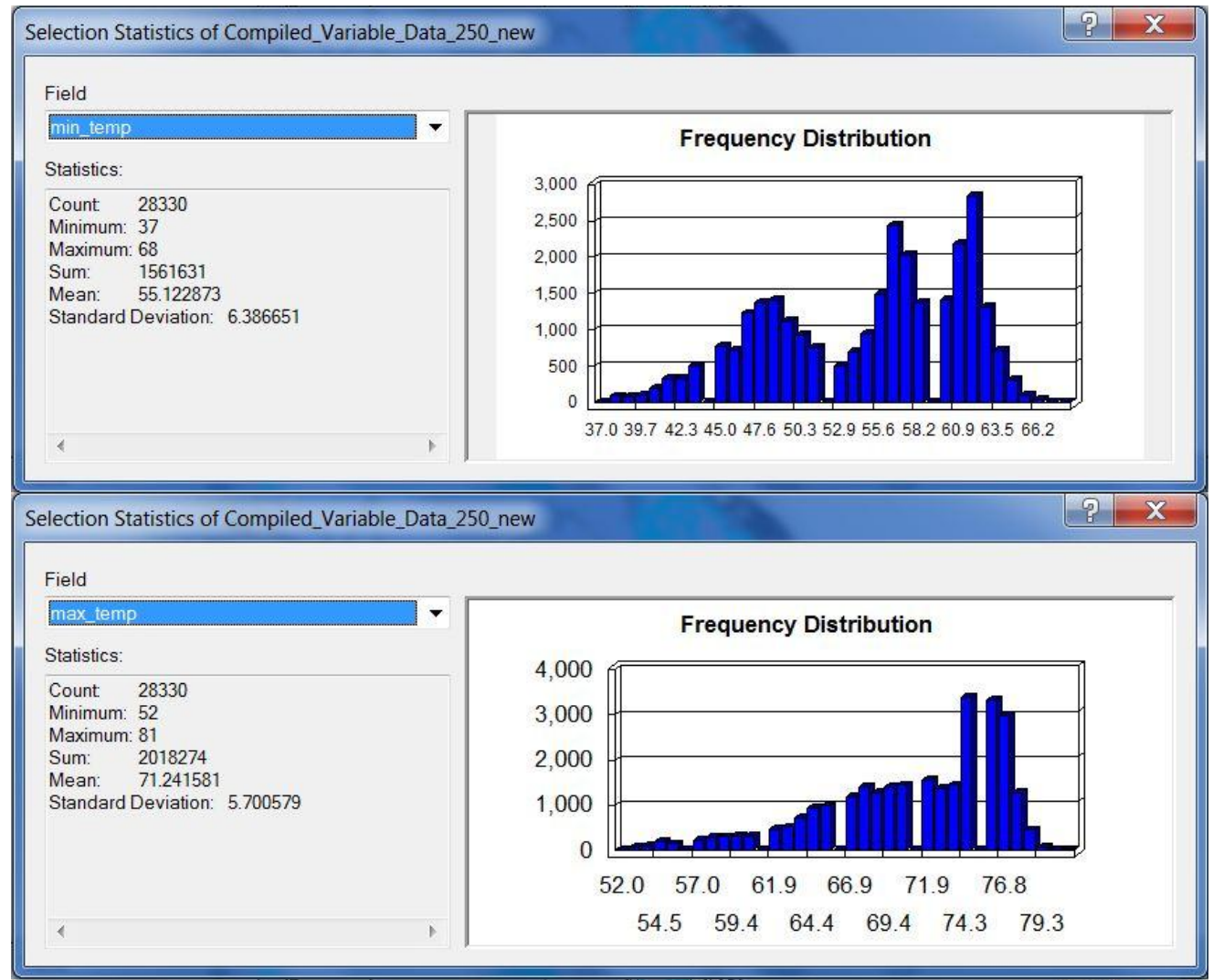

\begin{tabular}{|l|l|l|}
\hline Selection Statistics of Compiled_Variable_Data_250_new & D & $X$ \\
\hline
\end{tabular}

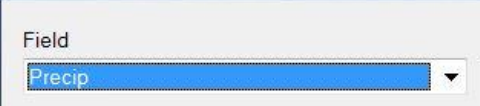

Statistics:

Count: 28330

Minimum: 34

Maximum: 213

Mean: $\quad 109.632016$

Standard Deviation: 56.039527

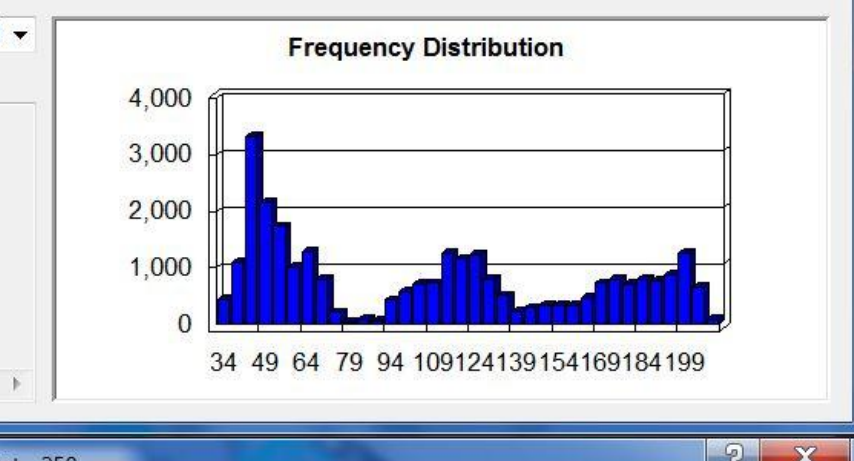

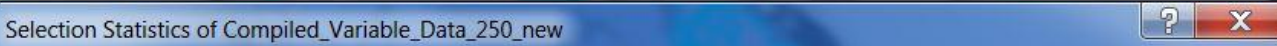

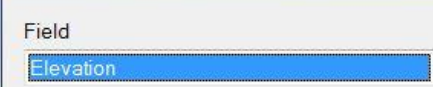

Statistics:

Count 28330

Minimum: 498.687665

Maximum: 7024.278226

Sum: $\quad 114756889.938211$

Mean: $\quad 4050.71973$

Standard Deviation: 1332.987691

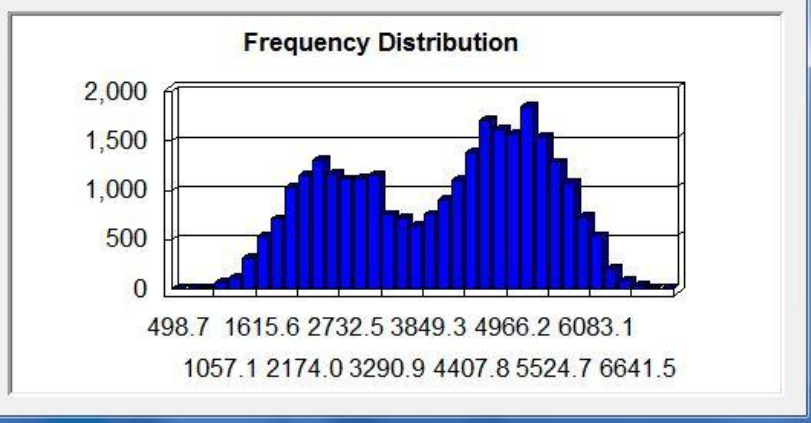

Figure A-8-3: Koa Forest Data Distributions 


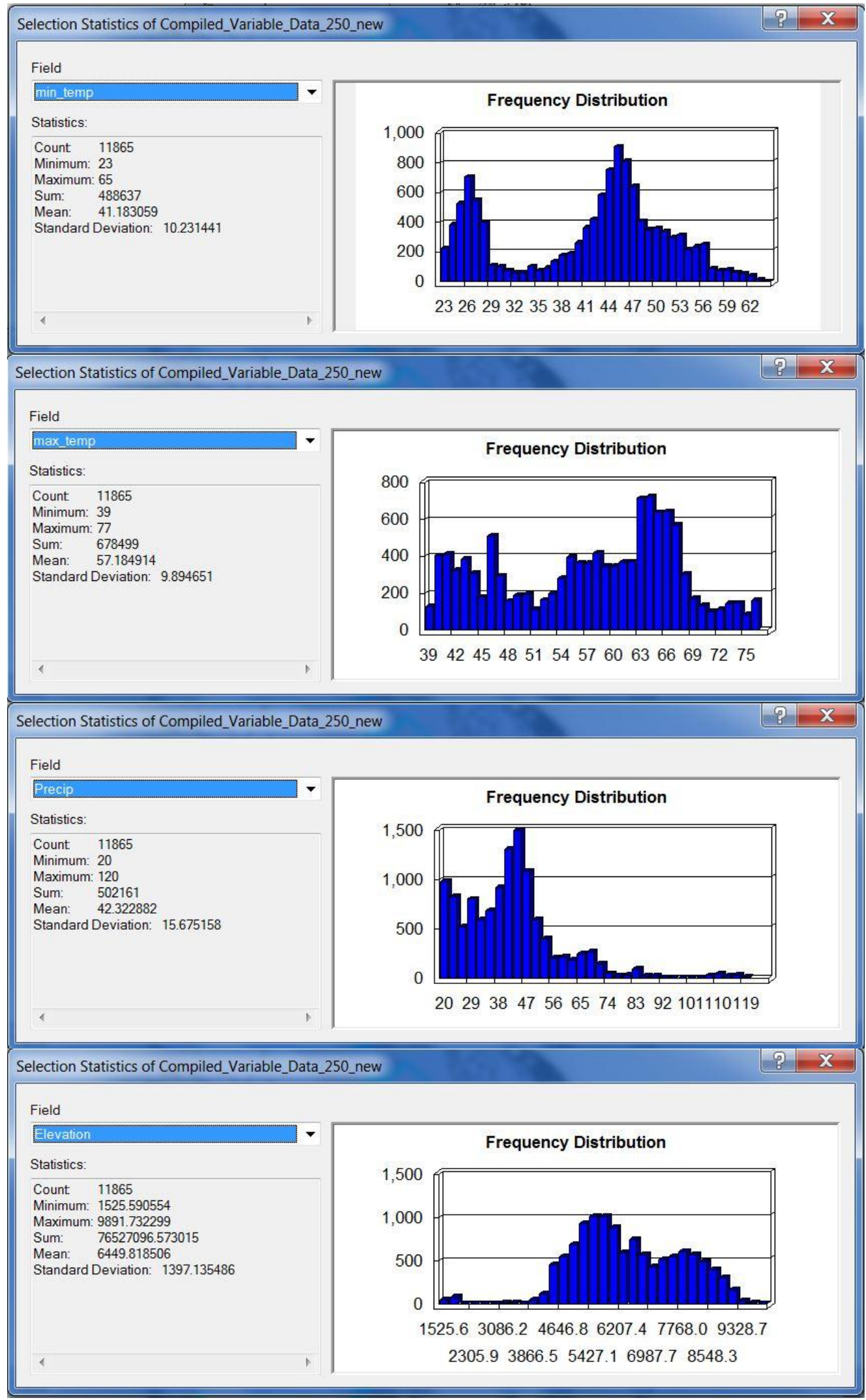

Figure A-8-4: Mamane Forest Data Distributions 

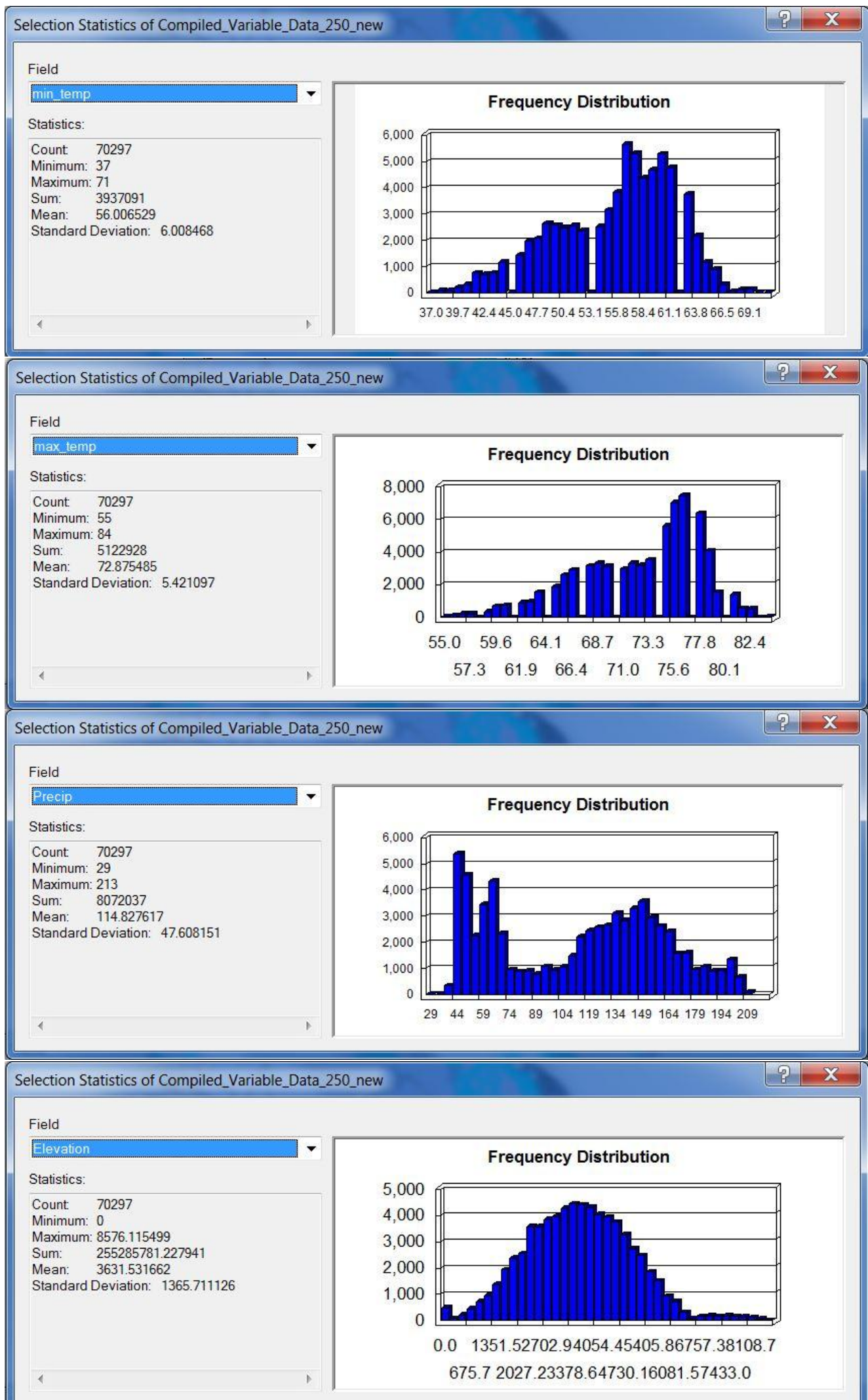

Figure A-8-5: Ohia Forest Data Distributions 